Modeled Neutron Induced Nuclear

Reaction Cross Sections for

Radiochemistry in the region of Iriduim and Gold

R. D. Hoffman, F. S. Dietrich, K. Kelley, J. Escher, R. Bauer, M. Mustafa

March 4, 2008 
This document was prepared as an account of work sponsored by an agency of the United States government. Neither the United States government nor Lawrence Livermore National Security, LLC, nor any of their employees makes any warranty, expressed or implied, or assumes any legal liability or responsibility for the accuracy, completeness, or usefulness of any information, apparatus, product, or process disclosed, or represents that its use would not infringe privately owned rights. Reference herein to any specific commercial product, process, or service by trade name, trademark, manufacturer, or otherwise does not necessarily constitute or imply its endorsement, recommendation, or favoring by the United States government or Lawrence Livermore National Security, LLC. The views and opinions of authors expressed herein do not necessarily state or reflect those of the United States government or Lawrence Livermore National Security, LLC, and shall not be used for advertising or product endorsement purposes.

This work performed under the auspices of the U.S. Department of Energy by Lawrence Livermore National Laboratory under Contract DE-AC52-07NA27344. 
UCRL-TR-401969

\title{
Modeled Neutron Induced Nuclear Reaction Cross Sections for Radiochemistry in the region of Iridium and Gold
}

\author{
R.D. Hoffman, F. S. Dietrich, K. Kelley, J. Escher, and R. Bauer \\ Nuclear Theory and Modeling Group \\ Physics and Advanced Technologies, N-Division \\ Lawrence Livermore National Laboratory \\ Livermore, CA 94550 \\ rdhoffman@llnl.gov \\ M. G. Mustafa \\ Nuclear and Defense Technologies, AX-Division \\ Lawrence Livermore National Laboratory \\ Livermore, CA 94550
}

August 18, 2008

\begin{abstract}
We have developed a set of modeled nuclear reaction cross sections for use in radiochemical diagnostics. Systematics for the input parameters required by the Hauser-Feshbach statistical model were developed and used to calculate neutron induced nuclear reaction cross sections for targets ranging from osmium $(Z=76)$ to gold $(Z=79)$. Of particular interest are the cross sections on Ir and Au including reactions on isomeric targets.
\end{abstract}

Subject headings: Nuclear cross sections, Radiochemistry, Nuclear Physics

\section{Introduction}

\subsection{Radiochemistry}

Various aspects of nuclear explosive device performance can be determined through the use of radiochemistry. During the UGT (Under Ground Test) Program, select naturally occurring elements were often loaded into a device prior to a test and their activation products subsequently retrieved for counting. The products are measured as isotopic ratios (such as ${ }^{87} \mathrm{Y} /{ }^{88} \mathrm{Y}$ produced from a stable isotope of the naturally occurring element). From the measured activity and prior knowledge of the amount of loaded detector material, performance aspects could be inferred by comparing the measured isotope ratios with those calculated using particle fluences from one of the design codes and group-averaged cross section sets that have been prepared for this purpose.

This paper continues the collaborative effort between AX-Division (WCI) and N-Division (PhySci) to update and improve the existing RADCHEM cross section detector sets. Previous papers treated the regions of bromine and krypton (Hoffman et al. 2004a), iodine and xenon (Hoffman et al. 2004b), samarium, europium, and gadolinium (Hoffman et al. 2004c), scandium, titanium, vanadium, chromium, manganese, and iron (Kelley et al. 2005), arsenic (Kelley et al. 2006a), nickel, copper, and zinc (Kelley et al. 2006b), and yttrium, zirconium, niobium, and molybdenum (Hoffman et al. 2006c). Here we focus on neutron-induced reactions proceeding on targets of iridium and gold. 


\section{Contents}

1 Introduction $\quad 1$

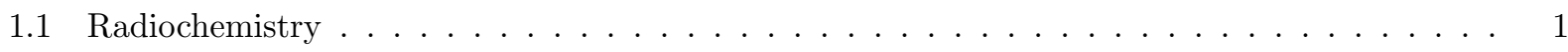

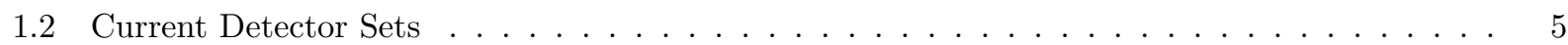

1.3 Motivation for Updating the Detector Sets . . . . . . . . . . . . . . . . . . . 5

1.4 Proposed Detector Sets $\ldots \ldots \ldots \ldots \ldots \ldots \ldots$

2 Nuclear Reaction Theory 6

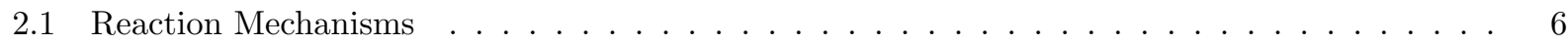

2.2 Hauser-Feshbach Statistical Model . . . . . . . . . . . . . . . . . . . 6

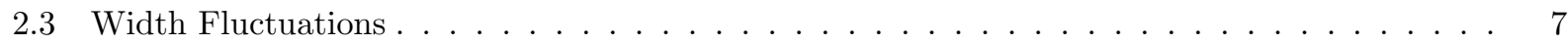

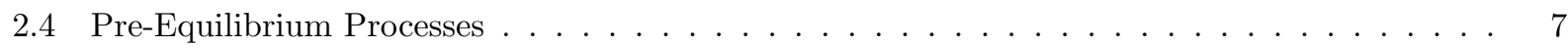

2.5 The STAPRE Hauser-Feshbach Reaction Code $\ldots \ldots \ldots \ldots \ldots \ldots$

3 Inputs to the Hauser-Feshbach Model $\quad 8$

3.1 Nuclear Structure Data . . . . . . . . . . . . . . . . . . . . . . . 8

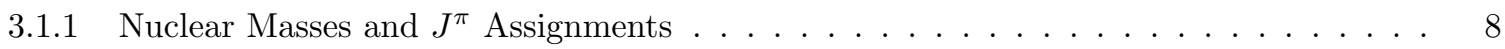

3.1 .2 Nuclear Level Schemes . . . . . . . . . . . . . . . . . . . . . . . . . . . . 8

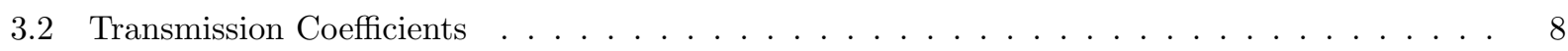

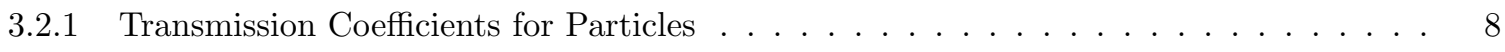

3.2.2 Considerations Regarding Collectivity and Nuclear Deformations . . . . . . . . . . 8

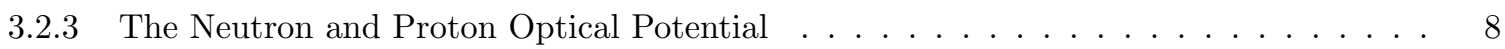

3.2.4 Evaluation of the Neutron and Proton Optical Potential . . . . . . . . . . . . . . . . 9

3.2.5 The Alpha and Deuteron Optical Potentials . . . . . . . . . . . . . . . . 10

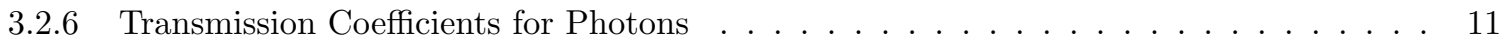

3.3 Nuclear Level Densities . . . . . . . . . . . . . . . . . . . . . . . . . . . . 13

3.3 .1 Level Density Models . . . . . . . . . . . . . . . . . . . . . . 13

3.3.2 Level Densities Above the Neutron Binding Energy . . . . . . . . . . . . . . . . . 14

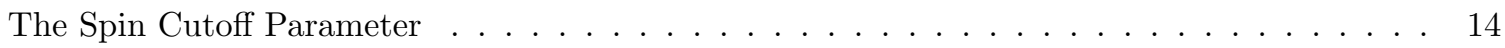

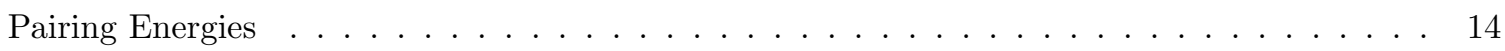

The Level Density Parameter . . . . . . . . . . . . . . . . . . . . . 14

3.3.3 Level Densities Below the Neutron Binding Energy . . . . . . . . . . . . . . . . 15

Behavior of the Spin Cutoff Parameter Below $E_{x} \ldots \ldots \ldots \ldots \ldots \ldots$

3.4 Considerations Regarding the Exciton Model . . . . . . . . . . . . . . . . 16

4 Modeled Cross Sections $\quad 17$

4.1 Comparison to Measured Cross Sections . . . . . . . . . . . . . . . . . 17

4.1.1 Comparison to experimental $(\mathrm{n}, \gamma)$ capture cross sections . . . . . . . . . . . 17

4.1.2 Comparison to Maxwellian averaged $(\mathrm{n}, \gamma)$ capture cross sections . . . . . . . . . 18

4.1 .3 Comparison to experimental (n,n') cross sections . . . . . . . . . . . . . . . 19

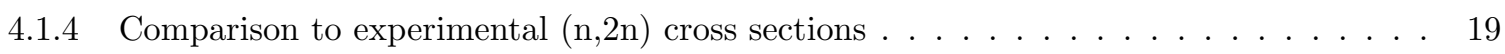

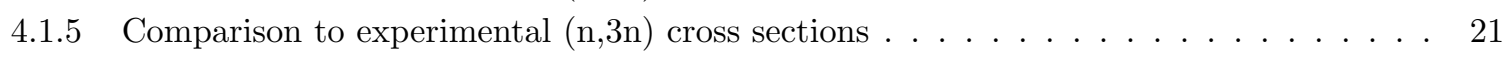

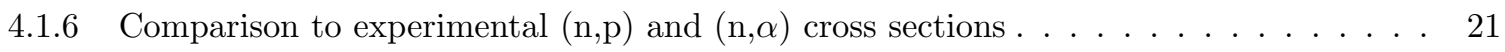

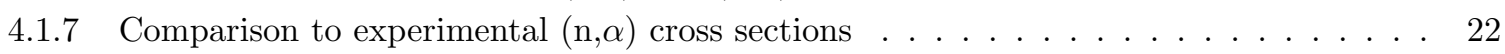

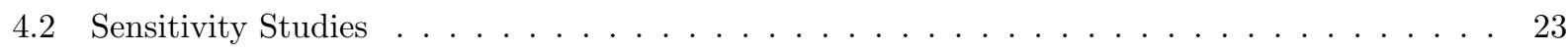

4.2.1 Sensitivity to the Pre-Equilibrium Cross Section $\ldots \ldots \ldots \ldots \ldots \ldots$ 
4.2.2 Sensitivity to the Alpha Pre-formation Parameter . . . . . . . . . . . . . . 24

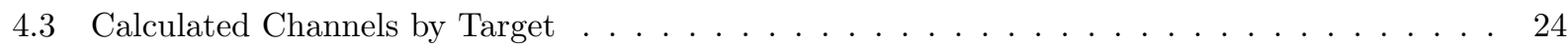

4.4 Comparison to LANL RADCHEM cross sections $\ldots \ldots \ldots \ldots \ldots \ldots \ldots \ldots$

5 Conclusions $\quad 25$

A Cross Sections Included in the Detector Sets $\quad 29$

A.1 Iridium and Gold Detector Sets . . . . . . . . . . . . . . . . . . . . . 29

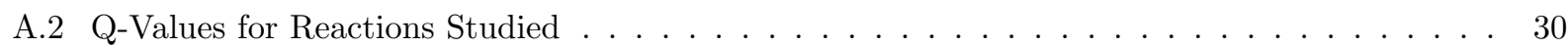

A.3 Modified Discrete Level Schemes _ . . . . . . . . . . . . . . . . . . . . 31

A.4 Level Density Parameters . . . . . . . . . . . . . . . . . . . . . . . . 43

B Modeled Cross Sections Compared to Measurements $\quad 46$

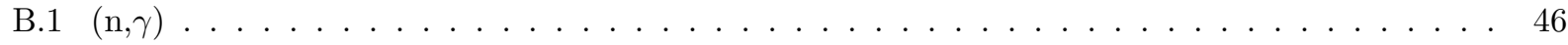

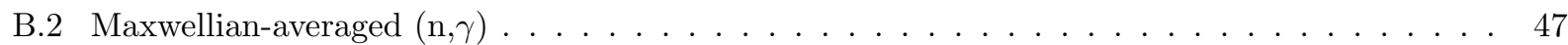

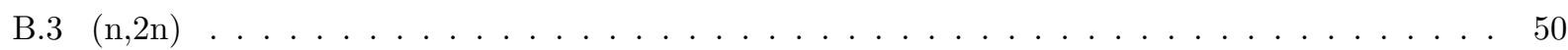

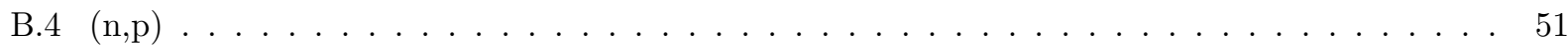

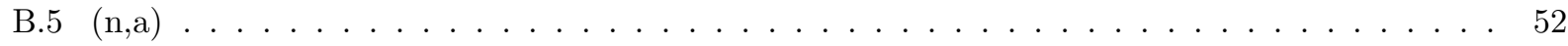

B.6 Comparison to LANL Radchem . . . . . . . . . . . . . . . . . . . . . . . . . . 53

C Activation Cross Sections by Target $\quad 55$

\section{List of Figures}

1 Collectivity in the region from Tungsten to Lead. . . . . . . . . . . . . . . . 8

2 Total measured neutron cross sections vs. FDOM Optical Potential for ${ }^{186} \mathrm{~W}+\mathrm{n}$ and ${ }^{197} \mathrm{Au}+\mathrm{n} . \quad 9$

3 Measured s- and p-wave strength functions and mean scattering radii vs. FDOM. . . . . . . . 10

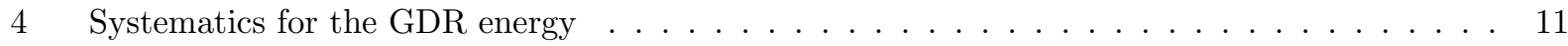

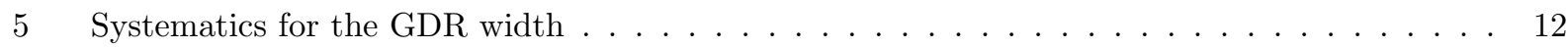

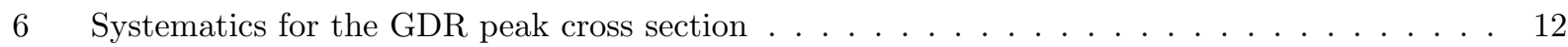

7 Systematics for average total s-wave radiation widths. $\ldots \ldots \ldots \ldots \ldots \ldots$

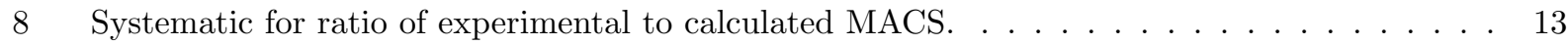

9 Three parameter fit to derived asymptotic level density parameters . . . . . . . . . . . . 15

10 Constant temperature level density fits to the low lying spectroscopic levels of ${ }^{198} \mathrm{Au} \ldots \ldots$. . 16

11 Calculated vs. measured $(\mathrm{n}, \gamma)$ cross sections $\ldots \ldots \ldots \ldots \ldots \ldots \ldots$

12 Calculated vs. recommended Maxwellian-averaged capture cross sections for ${ }^{191,193} \mathrm{Ir}$ and ${ }^{197} \mathrm{Au} 19$

13 Modeled (n,n') cross sections compared to measurement . . . . . . . . . . . . . . 20

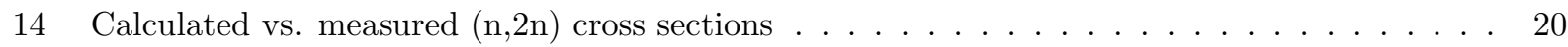

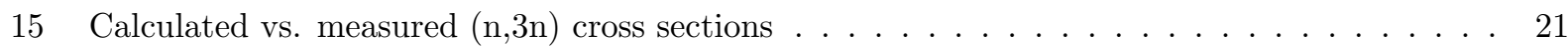

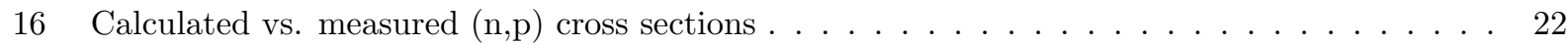

17 Calculated vs. measured $(\mathrm{n}, \alpha)$ cross sections $\ldots \ldots \ldots \ldots \ldots \ldots \ldots \ldots$

18 Sensitivity of ${ }^{197} \mathrm{Au}$ activation cross section to the alpha pre-formation parameter . . . . . . 23

19 Adopted level schemes for ${ }^{185} \mathrm{Ir}$ and ${ }^{186} \mathrm{Ir} \ldots \ldots \ldots \ldots \ldots \ldots \ldots \ldots$

20 Adopted level schemes for ${ }^{187} \mathrm{Ir}$ and ${ }^{188} \mathrm{Ir} \ldots \ldots \ldots \ldots \ldots \ldots$

21 Adopted level schemes for ${ }^{189} \operatorname{Ir}$ and ${ }^{190} \operatorname{Ir}(3 ; 4 ; 5) \ldots \ldots \ldots \ldots \ldots \ldots$

22 Adopted level schemes for ${ }^{191} \operatorname{Ir}$ and ${ }^{192} \operatorname{Ir}(1) \ldots \ldots \ldots \ldots \ldots \ldots \ldots$

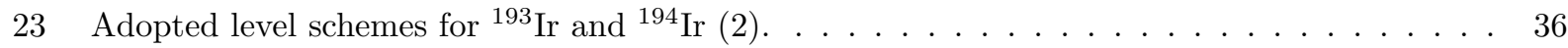

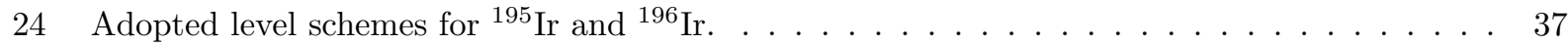


Adopted level schemes for ${ }^{191} \mathrm{Au}$ and ${ }^{192} \mathrm{Au}$. . . . . . . . . . . . . . . . . . . . . 38

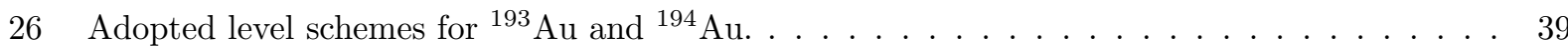

27 Adopted level schemes for ${ }^{195} \mathrm{Au}$ and ${ }^{196} \mathrm{Au}$. . . . . . . . . . . . . . . . . . . . . . . 40

28 Adopted level schemes for ${ }^{197} \mathrm{Au}$ and ${ }^{198} \mathrm{Au}$. . . . . . . . . . . . . . . . . . . . . . . 41

29 Adopted level schemes for ${ }^{199} \mathrm{Au}$ and ${ }^{200} \mathrm{Au}$. . . . . . . . . . . . . . . . . . . . . . . . 42

30 Modeled neutron capture cross sections compared to measurement . . . . . . . . . . . . . . 46

31 Modeled Maxwellian-averaged neutron capture cross sections compared to measurement . . . 47

32 Modeled $(\mathrm{n}, 2 \mathrm{n})$ cross sections compared to measurement . . . . . . . . . . . . . . . . . 50

33 Modeled $(\mathrm{n}, \mathrm{p})$ cross sections compared to measurement . . . . . . . . . . . . . . . 51

34 Modeled $(\mathrm{n}, \alpha)$ cross sections compared to measurement . . . . . . . . . . . . . . . . 52

35 Modeled cross sections for ${ }^{191}$ Ir compared to measurement and their LANL counterparts . . . 53

36 Modeled cross sections for ${ }^{193}$ Ir compared to measurement and their LANL counterparts . . . 54

37 Activation cross sections for targets of $\mathrm{Ir}$. . . . . . . . . . . . . . . . . . . . . . . 55

38 Activation cross sections for targets of $\mathrm{Au}$. . . . . . . . . . . . . . . . . . 56

\section{List of Tables}

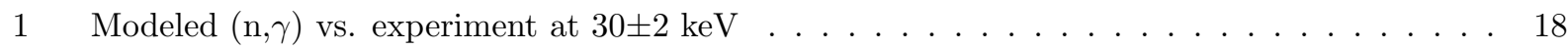

2 Comparison of Maxwellian-averaged $(n, \gamma)$ cross sections . . . . . . . . . . . . . . . . . . . 18

3 Comparison of our modeled $(\mathrm{n}, 2 \mathrm{n})$ cross sections to experimental data at $14.7 \pm 0.1 \mathrm{MeV}$ for activation (A), ground state (GS) and isomer (M1) targets. . . . . . . . . . . . . . 21

4 Sensitivity of select $14.7 \mathrm{MeV}(\mathrm{n}, 2 \mathrm{n})$ cross sections to the $\langle F M\rangle$ parameter . . . . . . . . . . 24

5 Neutron induced reactions calculated for new iridium and gold sets . . . . . . . . . . . . . . . 29

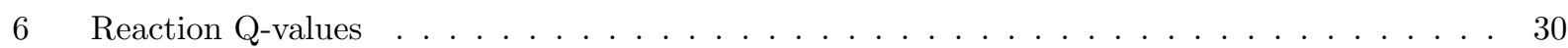

7 Level Density Parameters . . . . . . . . . . . . . . . . . . . . . . . . 4 43 


\subsection{Current Detector Sets}

Over the last 40 years a number of detector sets have been developed at LLNL and LANL. Twenty-three neutron threshold detector sets and five charged particle sets are currently available. The sets of interest in this modeling effort are as follows:

- Iridium neutron-induced set (Ir0188), used to calculate the production of ${ }^{189} \operatorname{Ir}\left(t_{1 / 2}=\right.$ $13.2 \mathrm{~d}),{ }^{190} \operatorname{Ir}\left(t_{1 / 2}=11.78 \mathrm{~d}\right),{ }^{192} \operatorname{Ir}\left(t_{1 / 2}=\right.$ $73.83 \mathrm{~d}),{ }^{193 m} \operatorname{Ir}\left(t_{1 / 2}=10.53 \mathrm{~d}\right)$, and ${ }^{194 m} \mathrm{Ir}$ $\left(t_{1 / 2}=171 \mathrm{~d}\right)$ from stable ${ }^{191,193} \mathrm{Ir}$.

- Gold neutron-induced set (Au1085), used to calculate the production of ${ }^{195} \mathrm{Au}\left(t_{1 / 2}=\right.$ $186.1 \mathrm{~d}),{ }^{196} \mathrm{Au}\left(t_{1 / 2}=6.17 \mathrm{~d}\right)$, and ${ }^{198} \mathrm{Au}$ $\left(t_{1 / 2}=2.696 \mathrm{~d}\right)$ from stable ${ }^{197} \mathrm{Au}$.

The cross sections available in these detector sets, as listed in (Nethaway 1998) are summarized in table 5 in appendix A. Most of the cross sections are taken from calculations performed at LLNL and LANL between 1985 and 1988. The iridium set was never finalized at LLNL due to incomplete or inaccurate information on discrete levels and branching ratios needed to accurately calculate the production of some important long-lived isomers. LANL has recently provided an updated evaluation (Chadwick et. al. 2007). For the gold set, the $(\mathrm{n}, 2 \mathrm{n})$ and $(\mathrm{n}, 3 \mathrm{n})$ reactions on stable ${ }^{197} \mathrm{Au}$ were based on evaluations of experimental data. Both these sets can be accessed on the world wide web at http://nuclear.llnl.gov/CNP/nads/main.html.

\subsection{Motivation for Updating the Detector Sets}

Many of the RADCHEM detector sets updated in our previous modeling efforts had a clear need for improvement. The major shortfalls of the Ir set was its preliminary nature mentioned above. We have made a thorough evaluation of the spectroscopic levels in Ir and calculated all the relevant cross sections using both ground state and long lived isomers as targets.

The historical cross section sets do not include charged particles in the exit channel of the neutron-induced reactions. A shortcoming of other historical cross section sets (e.g. Y), was that the remaining reaction channels (in $\mathrm{Y}$ ) were lumped together as a so-called $(\mathrm{n}, \mathrm{X})$ "destruction" cross section. In several instances, the $(\mathrm{n}, \mathrm{X})$ cross section is significantly larger than the channels explicitly calculated. Here, because these targets have such a high $\mathrm{Z}$ value, charged particles are strongly inhibited by the Coulomb barrier in the exit channel, so the dominant reactions will only involve neutrons and photons.

Another motivation is drawn from the general improvement in cross section modeling capabilities. In the nearly two decades since these sets were developed, many new cross section measurements have been performed, and the amount of nuclear structure data used to constrain model parameters has increased. Additionally, several efforts have been made to develop consistent approaches to modeling nuclear reaction cross sections (Belgya et al. 2005), and there are more accurate methods of calculating and estimating cross sections for which we have no data.

Thirdly, our proposed new evaluation of these cross sections includes an in-depth investigation into the sensitivity of the modeled cross sections to variations in the various statistical model inputs. In doing so we are able to determine which parameters are the most important for a given reaction. This also allows us to estimate how much a calculated cross section will change if new experimental measurements place more constraints on the model inputs.

\subsection{Proposed Detector Sets}

We consider as targets each of the isotopes listed in Table 5 of Appendix A. For each of these targets, we model the reaction channels indicated in the table. In cases where the residual nucleus has a long-lived isomer (which we define as $t_{1 / 2}>1 \mu \mathrm{s}$ ), we model individual cross sections leading to the ground and isomeric states, as well as an "activation" cross section, defined as the total cross section producing a given isotope. This modeling effort includes all of the reactions previously available in the RADCHEM detector sets, but also includes many additional targets and reaction channels. These additional reactions are included primarily to provide further comparisons to measured cross section data and bolster our confidence in the accuracy of cross sections modeled for unstable targets. These reactions also account for the various possible destruction reactions that are significant in this mass range.

Our goal is to develop a consistent set that reproduces, as closely as possible, measured cross sections on targets in the local region of interest. To do this we develop local systematics for the many input quantities used in the theoretical reaction modeling calculations. These systematics 
are based on experimental data that are often only available for compound nuclear systems formed from a stable target plus a neutron. Of course, we use experimental data whenever it is available, but reactions proceeding through unstable systems are unavoidable in radiochemistry. Short of developing new experimental techniques to measure cross sections on unstable targets, our only hope of reproducing measured activity from UGT shots, and addressing the uncertainty associated with the nuclear cross sections, is to develop cross section sets that reproduce well the measured cross sections in the local region of interest.

In $\S 2$ we describe the theoretical techniques used in the modeling effort. $\S 3$ describes the input parameters. $\S 4$ gives results. We conclude with $\S 5$.

\section{Nuclear Reaction Theory}

\subsection{Reaction Mechanisms}

Conceptually, we consider nuclear reaction mechanisms to be of two general types: direct processes and compound processes. Direct processes can be pictured as simple interactions of the incident particle with the nuclear potential of the target nucleus. They proceed on a rapid time scale (of order $\sim 10^{-22} \mathrm{~s}$ ), and the reaction products are often highly peaked in the incident particle direction. Direct reactions are generally quite small over the energy range of interest in this study, and have not been included in our calculations.

Compound processes are pictured as complicated interactions proceeding over a much longer timescale $\left(10^{-15}-10^{-18} \mathrm{~s}\right)$ in which the reaction is mediated by the formation of a "compound nucleus", with the excitation energy of the incident particle being statistically "shared" with the ensemble of nucleons in the target over all energetically allowed degrees of freedom. The reaction products are largely isotropic.

Other intermediate reaction mechanisms exist between these two extremes. We refer to these as "pre-equilibrium" nuclear processes, where a particle may be emitted from the target+projectile compound system prior to equilibration. Over the energy range of interest to this project (a few $\mathrm{keV}$ to $20 \mathrm{MeV}$ ) we will consider pre-equilibrium and compound nuclear processes, with the preequilibrium processes operating principally above $10 \mathrm{MeV}$ of incident particle energy.

\subsection{Hauser-Feshbach Statistical Model}

A traditional theoretical approach to compound nuclear reactions is the statistical or HauserFeshbach model (Hauser \& Feshbach 1952). This model is valid for high level densities in the compound nucleus, allowing one to use energy averaged transmission coefficients $T$, which describe absorption via an imaginary part in the (optical) nucleon-nucleus potential (Mahaux \& Weidenmüller 1979). For the reaction I (in state $\mu$ ) $+j \rightarrow k+\mathrm{L}$ (in state $\nu$ ), with $\mathrm{I}^{\mu}+j$ interacting with center-of-mass energy $\mathrm{E}_{j}^{\mu}$ (in $\mathrm{MeV}$ ), the average cross section is given by

$$
\sigma_{j k}^{\mu \nu}\left(E_{j}^{\mu}\right)=\frac{\pi \lambda_{j}^{2}}{g_{I}^{\mu} g_{j}} \sum_{J, \pi} g_{J} \frac{T_{j}^{\mu}\left(J^{\pi}\right) T_{k}^{\nu}\left(J^{\pi}\right)}{T_{t o t}\left(J^{\pi}\right)} W\left(J^{\pi}\right)
$$

where the summation extends over all compound nuclear spins and parities $J^{\pi}, \mu$ and $\nu$ enumerate states in the target and product $(=0$ for the ground state, 1 for the $1^{\text {st }}$ excited state, etc.). The cross section has units of area, described by $\pi \lambda_{j}^{2}=$ $0.6566\left(\hat{A}_{j} E_{j}^{\mu}\right)^{-1}$ barns, with $\hat{A}_{j}=\left(A_{I} A_{j}\right) /\left(A_{I}+\right.$ $A_{j}$ ) being the reduced mass in atomic mass units and $E_{j}^{\mu}$ is the center of mass energy in units of $\mathrm{MeV} . \lambda_{j}$ is the wavelength related to the wave number $k_{j}$ in the target plus incident particle channel by $\lambda_{j}=1 / k_{j}$ The statistical weights are given by $g_{y}^{x}=\left(2 J_{y}^{x}+1\right)$. Items without superscripts refer to the compound nucleus.

The transmission coefficients in the numerator are given by $T_{j}^{\mu}\left(J^{\pi}\right)=$ the total transmission coefficient for forming the state $J^{\pi}$ in the compound nucleus $I^{\mu}+j$ at energy $E_{j}^{\mu}$. Likewise, $T_{k}^{\nu}\left(J^{\pi}\right)$ is the same as $T_{j}^{\mu}\left(J^{\pi}\right)$ but for the pair $L^{\nu}+k$ at energy $E_{k}^{\nu}$. Implicit in these definitions is a sum over all possible $l$-waves and channel spins, i.e.

$$
T_{j}^{\mu}\left(J^{\pi}\right)=\sum_{l, s} T_{j}^{\mu}\left(J^{\pi}, l, s\right)
$$

where $l$ is any partial wave number (orbital angular momentum) that can couple the state $\mu$ to the compound nuclear state having spin and parity $J^{\pi}$ subject to quantum mechanical selection rules and $s$ is the vector sum of the spins $J_{I}^{\mu}$ and $J_{j}$. Hence $s$ takes on all integer (or half-integer) numbers from $\left|J_{I}^{\mu}-J_{j}\right|$ to $J_{I}^{\mu}+J_{j}$.

$T_{\text {tot }}$ represents the sum of transmission coefficients over all possible decay channels (i.e. for all particles and photons). The cross section for the formation of species L, regardless of its state 
$\nu$, is obtained by summing Eq. [1] over all bound states $\nu$ of $\mathrm{L}$ for which the reaction is energetically allowed.

When evaluating these sums, if energies become of interest which exceed the highest discrete excited state for which energy, spin, and parity are explicitly known, a nuclear level density formula must be employed. Specifically, the definitions for the transmission coefficients $T_{j}\left(J^{\pi}\right), T_{k}\left(J^{\pi}\right)$, and $T_{\text {tot }}\left(J^{\pi}\right)$ must be modified:

$$
\begin{aligned}
& T_{k}\left(J^{\pi}\right)=\sum_{\nu=0}^{\omega} T_{k}^{\nu}\left(J^{\pi}\right)+ \\
& \sum_{J^{\nu} \pi^{\nu}} \int_{\xi_{L}^{\omega}}^{\xi_{L}^{\text {max }}} T_{k}^{\nu}\left(\xi_{L}^{\nu}, J^{\pi}\right) \rho\left(\xi_{L}^{\nu}, J^{\nu}, \pi^{\nu}\right) d \xi_{L}^{\nu} d \pi^{\nu} d J^{\nu}
\end{aligned}
$$

where for the nucleus $\mathrm{L}, \xi_{L}^{\omega}$ is the energy of the highest excited state, $\omega$, of known energy, spin, and parity; $\xi_{L}^{\max }=E_{k}^{0}=E_{j}^{0}+Q_{j k}$ is the maximum excitation energy available, and $\rho\left(\xi_{L}^{\nu}, J^{\nu}, \pi^{\nu}\right)$ is the density of states per unit energy of spin and parity $J^{\nu}$ and $\pi^{\nu}$ at the excitation energy $\xi_{L}^{\nu}$. The above integral approximates a sum and is subject to the same quantum mechanical restrictions implied in the definition of the transmission function.

\subsection{Width Fluctuations}

In addition to the ingredients required for Eq. [1], we apply width fluctuation corrections $\left(W\left(J^{\pi}\right)\right.$, hereafter WFC), which define correlation factors with which all partial channels of incoming particle $j$ and outgoing particle $k$, passing through excited state $(E, J, \pi)$, should be multiplied. The major effect is to enhance the elastic channel and accordingly decrease the other open channels. They are most often observed at or near channel opening energies, for example when a $(\mathrm{p}, \gamma)$ and a $(\mathrm{p}, \mathrm{n})$ channel compete and the weaker $(\mathrm{p}, \gamma)$ channel is enhanced. Above a few $\mathrm{MeV}$ of excitation energy, when many competing channels are open, WFC's can be neglected.

A reasonably complete treatment for the WFC, obtained with the Gaussian orthogonal ensemble (GOE) approach, requires the evaluation of a triple integral and to date has been considered much to costly to apply in nuclear cross section calculations. Several approximations have been developed, the most popular ones are the Moldauer model (Moldauer 1976), and the HRTW model (Hofmann et al. 1975). We use the Moldauer model approximation in this study. For a detailed description of the full (GOE) treatment and a comparison with the Moldauer and HRTW approximation models mentioned above, see (Hilaire Lagrange \& Koning 2003).

\subsection{Pre-Equilibrium Processes}

For excitation energies starting around 10 $\mathrm{MeV}$, pre-equilibrium processes become important. The pre-equilibrium cross section is subtracted from the total reaction cross section leading to the first compound nucleus, and is usually unimportant for subsequent compound nuclei. Here we describe equilibration of the compound nuclear system in terms of the exciton model (Cline \& Blann 1971) including alpha particle emission (Milazzo-Colli \& Braga-Marcazzan 1973). We adopt an initial 2-particle 1-hole configuration. Average rates for internal transitions, corrected for the Pauli principle by (Cline 1972), are related by the formulas of (Williams 1970) to the absolute square of the average effective matrix element $|M|$ of the residual interactions as per Eq. [7] of (Uhl \& Strohmaier 1976). The dependence of $|M|^{2}$ on mass number and excitation energy is

$$
|M|^{2}=\langle F M\rangle A^{-3} E^{-1}
$$

The description of alpha particle emission in the pre-equilibrium model is a straightforward extension of nucleon emission, assuming nucleons preform alpha clusters. In making such an extension, one introduces a parameter $\phi$ which represents the probability that the incoming particle will strike a pre-formed alpha cluster.

In the pre-equilibrium stage of the reaction, particle emission is assumed to be the only decay mode. For the equilibration, the WFC corrected Hauser Feshbach formula (Eq. [1]) is applied. All subsequent processes are treated as sequential evaporation steps.

\subsection{The STAPRE Hauser-Feshbach Reac- tion Code}

We model our cross sections using the statistical model code STAPRE (Uhl \& Strohmaier 1976), which embodies all of the physical models discussed above. The version of the code we use is STAPRE-H95 (Avrigeanu \& Avrigeanu 1976), available from the NEA web site. We have made several modifications, primarily to the level density routines. Prior versions of the code were used to develop parts of the existing RADCHEM data sets (Vonach 1982). 
In the following we discuss the important ingredients of statistical model calculations, and the methods utilized to estimate them. These are the requisite nuclear structure data, such as the binding energies of all nuclei included (which define the separation and reaction threshold energies and Q-values of the various reaction channels considered), as well as the energies, spins, and parities of the ground states and all known excited states of these nuclei, and the detailed branching ratios for the gamma-ray cascade from excited to lowlying states. Also needed are parameters controlling the width fluctuation corrections and the preequilibrium model, the particle and $\gamma$-transmission coefficients, and the nuclear level densities of all nuclei involved in a given reaction. The reliability with which these ingredients can be calculated determines the accuracy (or reliability) of a given cross section calculation.

\section{Inputs to the Hauser-Feshbach Model}

\subsection{Nuclear Structure Data}

\subsubsection{Nuclear Masses and $J^{\pi}$ Assignments}

We adopt for nuclear masses the experimental mass excess values (Wapstra et al. 2003; Audi et al. 2003). Spin and parity assignments for the ground state and isomer targets (Tuli 2000) are given in Table 5 (Appendix A). In Table 6 (Appendix A.2) we provide reaction Q-values for the cross sections modeled in this study.

\subsubsection{Nuclear Level Schemes}

The nuclear structure data used to model the gamma-ray cascade was adopted from many sources, starting with (Belgya et al. 2005). All schemes were exhaustively evaluated (Bauer \& Kelley 2007 The modified nuclear level schemes for the Iridium and Gold, providing the level energies, spin and parity assignments, and $\gamma$-ray branching ratios, and the main references used to evaluate them may be found in appendix A.3. The number of excited levels adopted for each nucleus is given as the quantity " $\mathrm{N}$ " in Table 7 (we always include a ground state, i.e. when $\mathrm{N}=0$ only the ground state is included).

\subsection{Transmission Coefficients}

\subsubsection{Transmission Coefficients for Particles}

Our modeling effort includes reactions with incident neutrons. To accurately calculate the exit

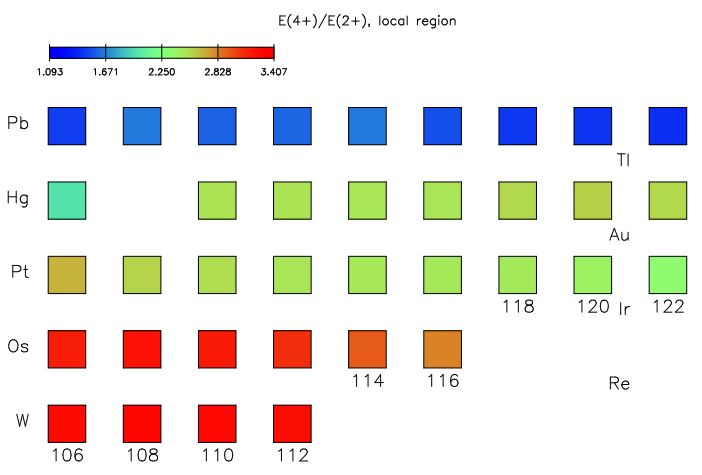

Fig. 1.- Collectivity in the region from Tungsten to Lead.

channel cross sections we also include transmission coefficients for protons, alpha-particles, and deuterons. For neutrons and protons, we develop our own optical model for deformed rare-earth nuclei. For alphas and deuterons, we adopt well established spherical optical models (see below).

\subsubsection{Considerations Regarding Collectivity and Nuclear Deformations}

Our region of interest extends from $108 \leq N \leq$ 122. To gauge the onset of collective effects we appeal to nuclear systematics. One such measure of "collectivity" is the ratio vs. neutron number $\mathrm{N}$ of the energy of the first $J^{\pi}=4^{+}$excited state to the first $J^{\pi}=2^{+}$excited state in even-Z even-N nuclei (Figure 1). Spherical (magic closed shell) nuclei exhibit collectivity near 1.6 and are clearly seen in the $\mathrm{Z}=82(\mathrm{~Pb})$ closed proton shell. Deformed vibrators occur between $2.0-2.4$ (all the -nuclei of $\mathrm{Hg}$ and $\mathrm{Pt}$ for $108 \leq \mathrm{N} \leq 122)$, followed by transitional nuclei up to 3.3 (W and Os), where a true rotational character is evident. The loaded ${ }^{191,193}$ Ir targets sit right in the transition zone between Os and Pt, while loaded ${ }^{197} \mathrm{Au}$ is clearly vibrational in character. This suggests we should adopt a deformed optical model for all our calculations, assuming a vibrational character for all nuclei up of $\mathrm{Au}$, and a rotational one for nuclei with $Z \leq 77$. We adopt the deformation parameters $(\beta)$ from the FDRM calculation of (Möller et al. 1995).

\subsubsection{The Neutron and Proton Optical Potential}

The optical potential used in determining the neutron and proton transmission coefficients (FDOM) was developed by F. Dietrich (Dietrich 2001). 
It is a deformed potential with a standard WoodsSaxon shape. The real volume potential has a depth of

$$
\begin{aligned}
V_{\text {vol. }}(E) & =(50.125-0.2331 E) \\
& -\eta_{p} \eta_{t}(20.050-0.0933 E) \\
& +\xi \frac{0.3 Z}{A^{1 / 3}}
\end{aligned}
$$

where

$$
\eta_{t}=\frac{A-2 Z}{A}
$$

and $\eta_{p}=1$ for incident neutrons and $\eta_{p}=-1$ for incident protons. Additionally, $\xi=1$ for incident neutrons and $\xi=0$ for incident protons. The real volume potential has a mean radius and diffusivity of $r_{v o l} .=1.25 A^{1 / 3}$ and $a_{v o l} .=0.65 \mathrm{fm}$, respectively.

The imaginary volume potential has a depth of

$$
\begin{aligned}
W_{\text {vol. }}(E) & =(-1.357+0.1696 E) \\
& -\eta_{p} \eta_{t}(-0.543+0.0678 E)
\end{aligned}
$$

When $W_{\text {vol. }}<0$, it is set to zero (i.e. we do not allow $W_{\text {vol. }}$. to become negative). The mean radius and diffusivity are the same as for the real volume potential.

The imaginary surface potential is broken into two parts. For incident energies less than $8 \mathrm{MeV}$, it has the form

$$
\begin{aligned}
W_{\text {surf. }}(E) & =(3.743+0.334 E) \\
& -\eta_{p} \eta_{t}(1.497+0.134 E)
\end{aligned}
$$

Above $8 \mathrm{MeV}$, we use

$$
\begin{aligned}
W_{\text {surf. }}(E) & =(6.974-0.0697 E) \\
& -\eta_{p} \eta_{t}(2.790-0.0279 E)
\end{aligned}
$$

As with the imaginary volume, we only use positive values for the surface potential, replacing negative values with zero. The mean radius is the same as for the volume terms, but the diffusivity is taken to be $a_{\text {surf. }}=0.58 \mathrm{fm}$.

Last of all we include a real spin-orbit potential with a depth of $8.427 \mathrm{MeV}$ with the same mean radius and diffusivity as the volume terms. Additionally, for incident protons, one must include the Coulomb potential.

The particle transmission coefficients were generated by the optical model code ECIS-95 (Raynal 1996), using a deformed potential. For $Z \geq 78$ we used a vibrational model, and for
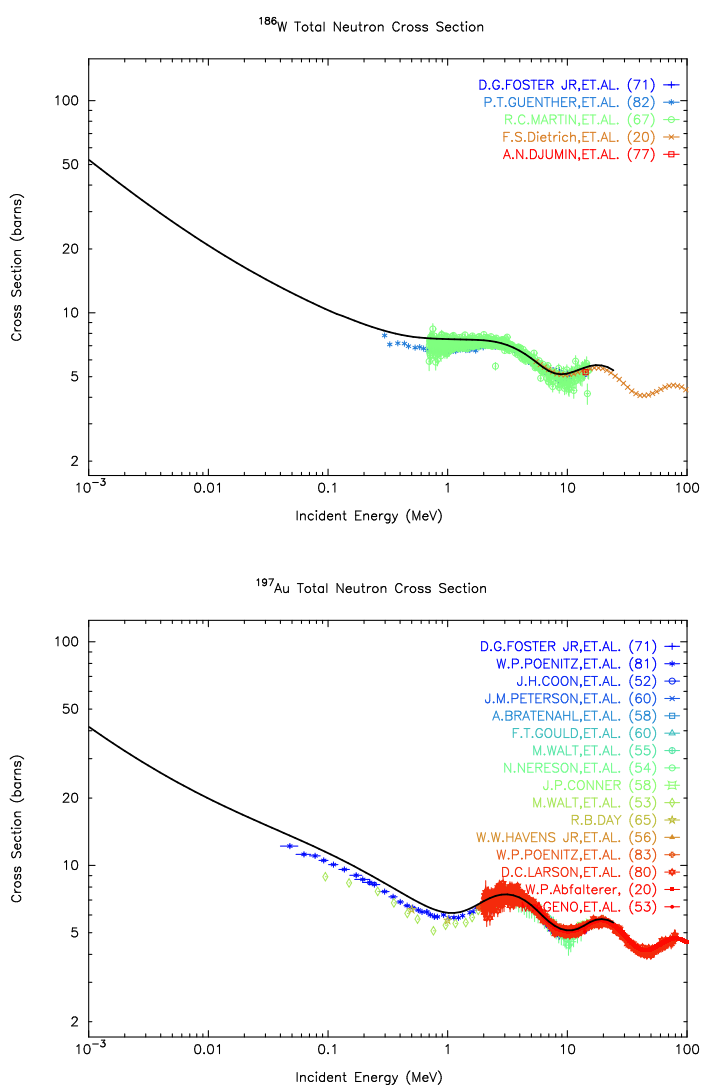

Fig. 2. - Total measured neutron cross sections vs. FDOM Optical Potential for ${ }^{186} \mathrm{~W}+\mathrm{n}$ and ${ }^{197} \mathrm{Au}+\mathrm{n}$.

$Z \leq 77$ we used a rotational model. For vibrational cases, we use a one-phonon model. For rotational cases, we allow up to quadrupole deformations. In either case, we include only one or two excited states. The states used are the ground state and first $J^{\pi}=2^{+}, 4^{+}$for even- $\mathrm{Z}$ even-N nuclei. For the remaining nuclei, a fictitious $0^{+}, 2^{+}$, $4^{+}$level scheme was developed. The energies for the levels were found by averaging the energies of the nearest even- $\mathrm{Z}$ even- $\mathrm{N}$ nuclei.

\subsubsection{Evaluation of the Neutron and Proton $\mathrm{Op}$ - tical Potential}

We present in Figure 2 results of the optical model compared to measured total neutron cross sections. The comparisons in Figure 2 are for total neutron cross sections on ${ }^{186} \mathrm{~W}$ and ${ }^{197} \mathrm{Au}$. Other experimental total neutron cross section data in this region generally consists of a single point at roughly $14 \mathrm{MeV}$, or several points below 10 $\mathrm{keV}$ of excitation energy. For cases with a sin- 
gle data point near $14 \mathrm{MeV}$, the optical model closely replicated the experimental data (typically within $10 \%$ ). No total proton cross section data was available in this region.

The comparison between the calculated total neutron cross sections from our rotational FDOM optical potential and available experimental data indicates a reasonable degree of agreement.

Further information regarding the quality of the neutron optical potential may be obtained by comparing the s- and $\mathrm{p}$-wave strength functions and mean scattering radii predicted by the model to measured values. We make such a comparison in Figure 3. Each of these plots show the ratio of the quantity predicted by the optical potential to the measured value, plotted against the mass of the compound (target plus neutron) system. The error bars reflect this same ratio using the upper and lower errors in the measured values. Hence, if the error bars cross unity, the optical model prediction is within the errors of the measured value. Our interest here is for $185 \leq A \leq 200$. For the swave strength functions $\left(S_{0}\right)$, we see that many of the optical model predictions lie within the errors of the measured values. In all cases, the modeled/measured ratio is with a factor of two (indicated by the two outer dotted lines). The predicted p-wave strength functions $\left(S_{1}\right)$, are slightly higher than a factor of two of the measured values. The mean scattering radii predicted by the optical potential $\left(R^{\prime}\right)$, are in very good agreement with the measurements.

\subsubsection{The Alpha and Deuteron Optical Poten- tials}

We have included possible alpha and deuteron exit channels (and appropriate transmission coefficients) in this modeling effort. For the alpha particles, we use the optical potential of (Avrigeanu et al. 1994), for deuterons we use (Lohr \& Haeberli 1974), as encoded in the spherical optical model subroutine SCAT2 of STAPRE.

We do not include a quality analysis of these potentials in this report. The deuteron and alpha exit channels are, in every case, very small when compared to the dominant channel, accounting for at most $1 \%$ of the total reaction cross section. Therefore, any sensitivity to the alpha and deuteron potentials will only be apparent in these weak exit channels which will have little or no impact on the analysis of UGT shots. Additionally, the relatively good agreement with the experimental $(\mathrm{n}, \alpha)$ cross sections provides us with some de-
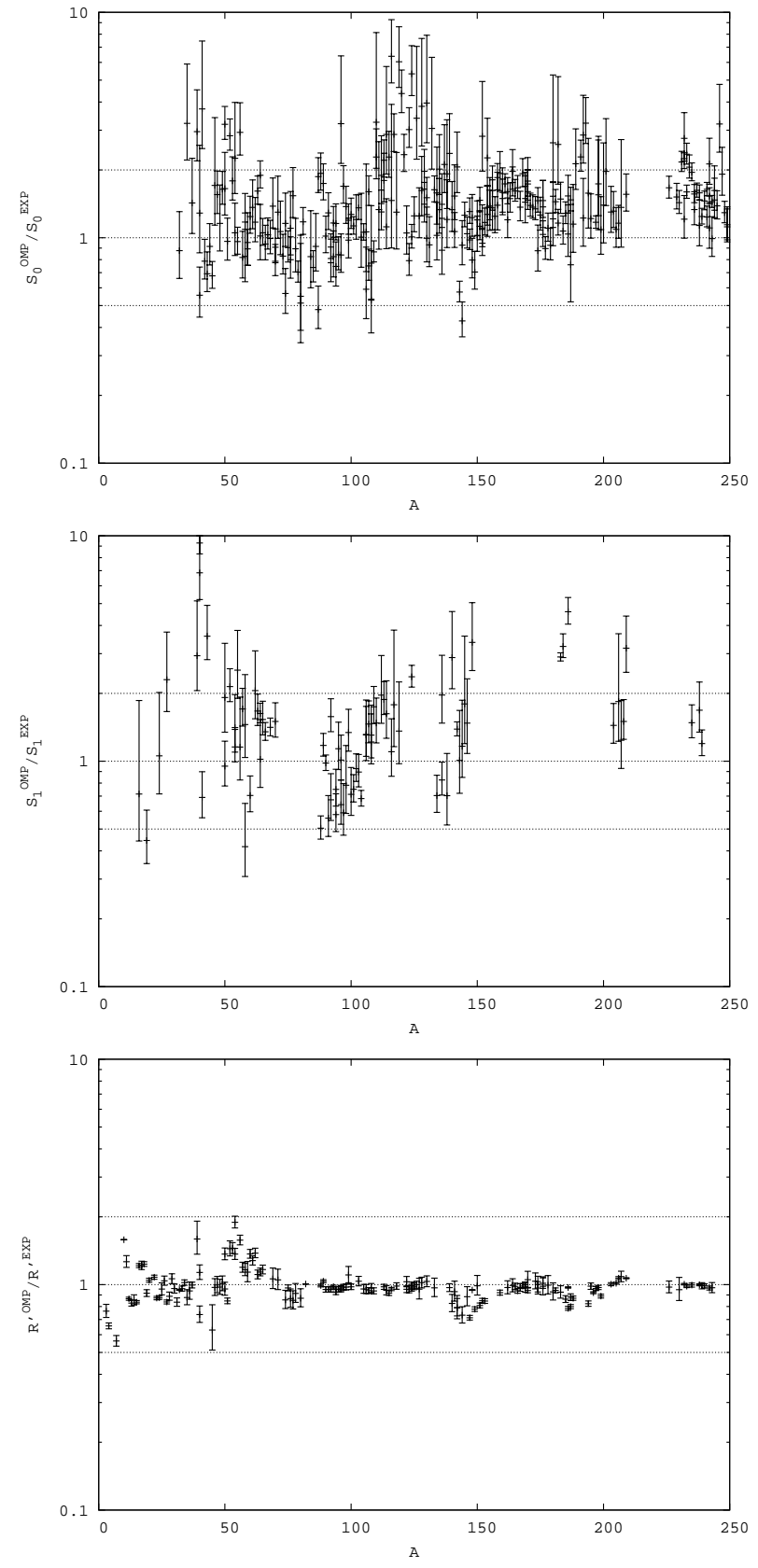

Fig. 3.- Measured s- and p-wave strength functions and mean scattering radii compared to the predictions of the rotational FDOM optical potential. The measured values for the s-wave and p-wave strength functions $\left(S_{0}\right.$ and $\left.S_{1}\right)$ are taken from (Belgya et al. 2005). Measured scattering radii are taken from (Mughabghab et al. 1981). Plotted are the ratios of the modeled quantities to their measured counterparts. The dotted lines indicate unity and factor of two deviations.

gree of confidence in the alpha potential (see Ap- 
pendix B).

\subsubsection{Transmission Coefficients for Photons}

Gamma ray transmission coefficients were calculated using a simple model which depends only on the multi-pole type (XL) and the transition energy $(\epsilon)$, as encoded in STAPRE. They are related to the gamma ray strength function $f_{X L}^{\gamma}(\epsilon)$ by

$$
T_{X L}^{\gamma}(\epsilon)=2 \pi \epsilon^{2 L+1} f_{X L}^{\gamma}(\epsilon)
$$

The energy dependence of the strength function was determined using the GDR model with enhanced generalized Lorentzian (EGLO) line shapes (Kopecky et al. 1993). In particular, the E1 strength function is given by

$$
\begin{gathered}
f_{E 1}^{\gamma}(\epsilon)=\mathcal{N} \frac{4}{3 \pi} \frac{e^{2}}{\hbar c} \frac{1}{M_{p} c^{2}} \times \\
{\left[\frac{\epsilon \Gamma_{G D R}\left(\epsilon, T_{f}\right)}{\left(\epsilon^{2}-E_{G D R}^{2}\right)^{2}+\left(\Gamma_{G D R}\left(\epsilon, T_{f}\right) \epsilon\right)^{2}}+\right.} \\
\left.0.7 \frac{\Gamma_{G D R}\left(0, T_{f}\right)}{\epsilon^{3}}\right]
\end{gathered}
$$

where $M_{p}$ is the proton mass. The energy dependent width $\Gamma_{G D R}\left(\epsilon, T_{f}\right)$ is given by

$$
\begin{aligned}
\Gamma_{G D R}\left(\epsilon, T_{f}\right)= & {\left[\kappa+(1-\kappa) \frac{\epsilon-\epsilon}{E_{G D R}-\epsilon}\right] \times } \\
& \frac{\Gamma_{G D R}}{E_{G D R}^{2}}\left[\epsilon^{2}+\left(2 \pi T_{f}\right)^{2}\right]
\end{aligned}
$$

with $\epsilon=4.5 \mathrm{MeV}$. For nuclei with $A<148$, the factor $\kappa$ is unity. For heavier nuclei, $\kappa=$ $1+0.009(A-148)^{2} \exp [-0.18(A-148)]$. The $T_{f}$ that appears in Equations 11 and 12 is the temperature of the final state, determined from the level density parameters. For a backshifted transition energy $U=S_{n}-\epsilon-\Delta$, one determines the energy dependent level density parameter (Eq. 28). Provided $U$ is positive, the temperature is given by

$$
T_{f}=\frac{a}{2}[1+\sqrt{1+4 a U}]
$$

Otherwise, $T_{f}=1 / a$.

Experimental values for two GDR resonances are available in this region (Belgya et al. 2005). Based on these measurements, we have adopted a systematic description of these parameters for other targets. Using only measured GDR parameters from the local region of interest, we developed the following systematic fits:

$$
E_{1}=6.42+0.03 \times A_{C} \mathrm{MeV}
$$
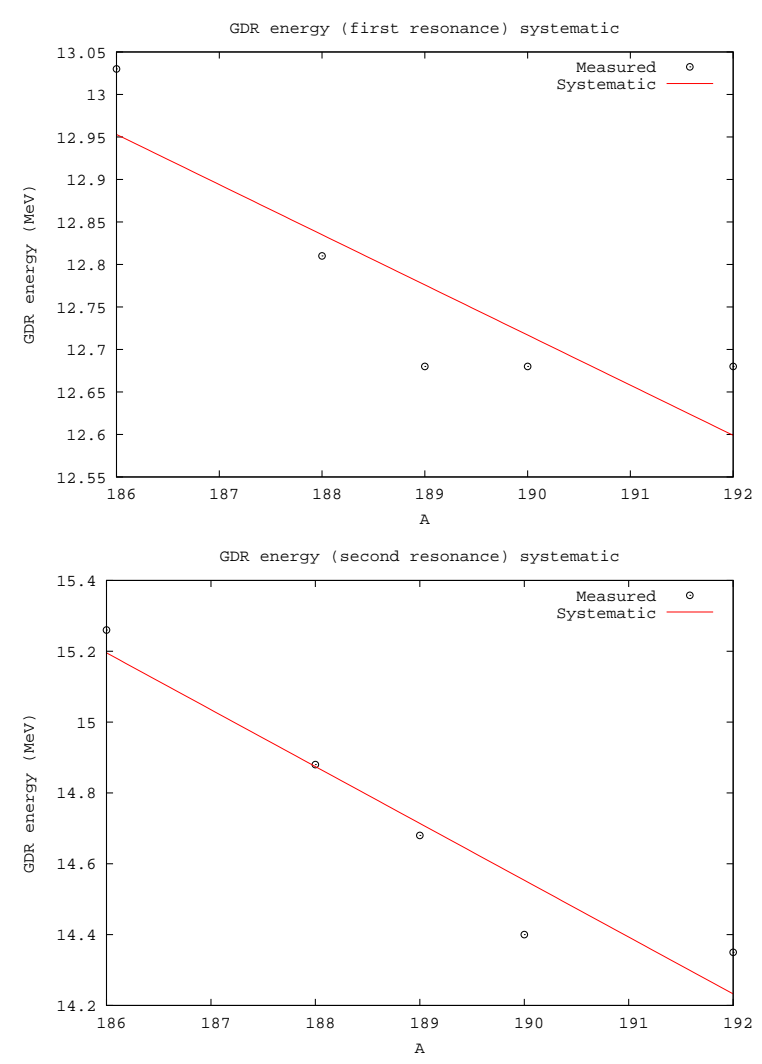

Fig. 4.- Systematics for the GDR energy, first resonance on the top panel, second on the bottom. The measured data in the local region of interest, indicated by black circles, are taken from (Belgya et al. 2005).

$$
\begin{aligned}
\Gamma_{1} & =2.95-0.002 \times A_{C} \mathrm{MeV} \\
\sigma_{1} & =-88.09+1.77 \times A_{C} \mathrm{mb} \\
E_{2} & =19.3-0.024 \times A_{C} \mathrm{MeV} \\
\Gamma_{2} & =5.03-0.004 \times A_{C} \mathrm{MeV} \\
\sigma_{2} & =-443.0+4.29 \times A_{C} \mathrm{mb}
\end{aligned}
$$

where $A_{C}$ is the mass number of the compound nucleus. The resulting systematic fits are presented in Figures (4-6).

We also include M1, E2, M2, E3, and M3 transitions in our modeling. For the M1 strength function, we adopt a Simple Lorentzian (SLO) model

$$
\begin{array}{r}
f_{M 1}^{\gamma}(\epsilon)=\mathcal{N}_{M 1} \frac{4}{3 \pi} \frac{e^{2}}{\hbar c} \frac{1}{M_{p} c^{2}} \times \\
\frac{\epsilon \Gamma_{G D R}}{\left(\epsilon^{2}-E_{G D R}^{2}\right)^{2}+\left(\Gamma_{G D R} \epsilon\right)^{2}}
\end{array}
$$

with the global set of GDR parameters given in 

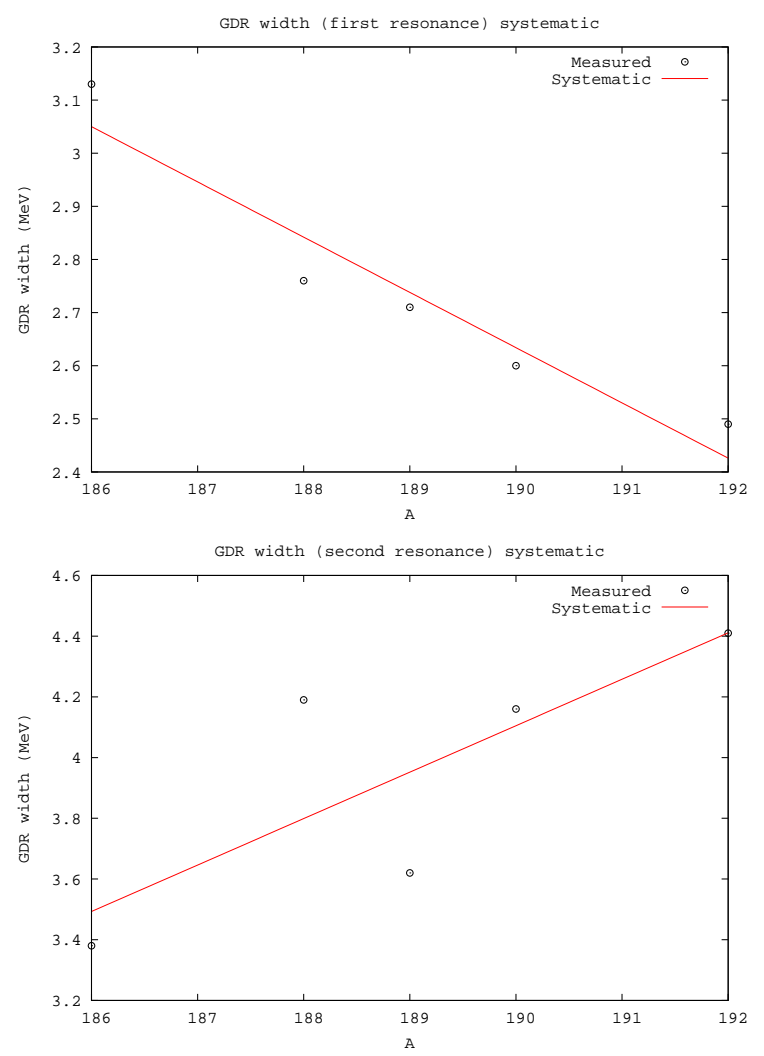

Fig. 5.- Systematics for the GDR width.

(Belgya et al. 2005):

$$
\begin{aligned}
E_{G D R} & =\frac{41}{A^{1 / 3}} \\
\Gamma_{G D R} & =4
\end{aligned}
$$

The overall normalization for the M1 strength function $\left(\mathcal{N}_{M 1}\right)$ is determined such that

$$
\frac{f_{E 1}\left(S_{n}\right)}{f_{M 1}\left(S_{n}\right)}=0.0588 \cdot A^{0.878}
$$

where $S_{n}$ is the neutron separation energy.

The remaining transmission coefficients are simply proportional to $\epsilon^{2 L+1}$, their strength functions are constants. In particular,

$$
\begin{aligned}
f_{E 2}^{\gamma}(\epsilon) & =7.2 \times 10^{-7} A_{C}^{2 / 3} f_{E 1}^{\gamma}\left(S_{n}\right) \\
f_{M 2}^{\gamma}(\epsilon) & =2.2 \times 10^{-7} f_{E 1}^{\gamma}\left(S_{n}\right) \\
f_{E 3}^{\gamma}(\epsilon) & =3.4 \times 10^{-13} A_{C}^{4 / 3} f_{E 1}^{\gamma}\left(S_{n}\right) \\
f_{M 3}^{\gamma}(\epsilon) & =1.1 \times 10^{-13} A_{C}^{2 / 3} f_{E 1}^{\gamma}\left(S_{n}\right)
\end{aligned}
$$

where $S_{n}$ is the neutron separation energy. In all cases, E1 is the dominant multipole.

The factor $\mathcal{N}$ appearing in equation 11 is a normalization constant, determined by fitting the average total s-wave radiation width at the neutron
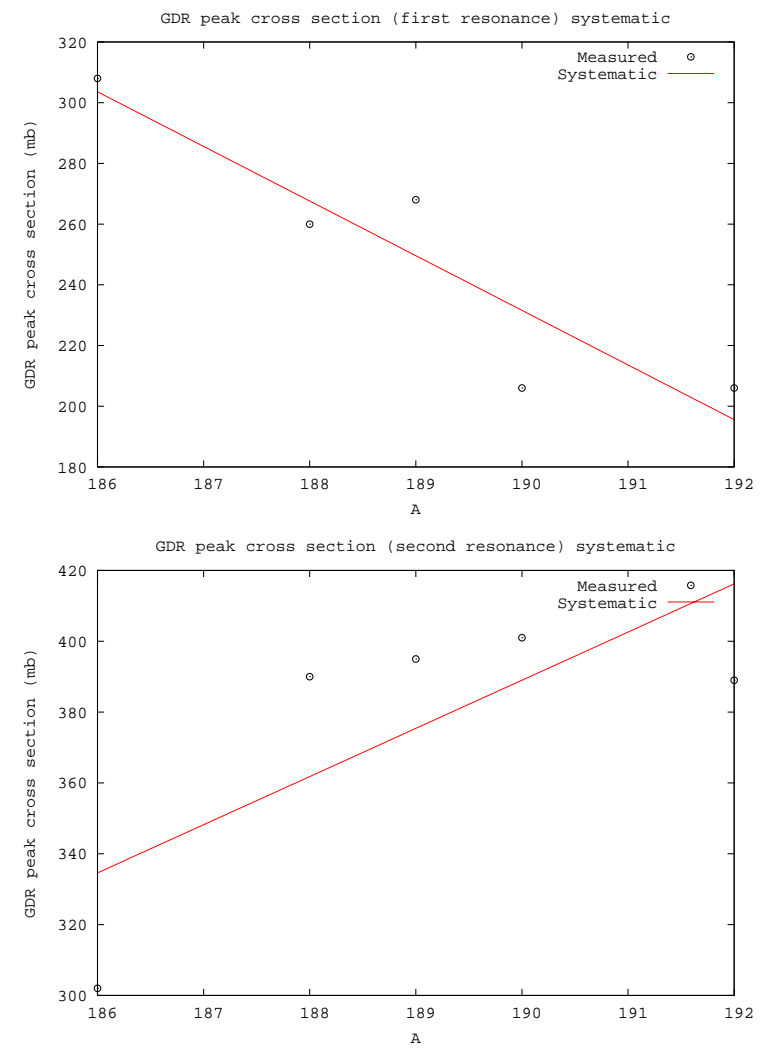

Fig. 6.- Systematics for the GDR peak cross section.

binding energy,

$$
\begin{gathered}
\left\langle\Gamma_{\gamma}\right\rangle_{0}=\frac{J+1}{2 J+1}\left\langle\Gamma_{\gamma}\left(B_{n}, J+\frac{1}{2}\right)\right\rangle \\
+\frac{J}{2 J+1}\left\langle\Gamma_{\gamma}\left(B_{n}, J-\frac{1}{2}\right)\right\rangle \\
\Gamma_{\gamma}(E, J)=\frac{T_{\gamma}(E, J)}{2 \pi \rho(E, J)}(\mathrm{meV})
\end{gathered}
$$

(Uhl \& Strohmaier 1976). Here, $J$ is the spin of the target nucleus. The gamma-ray transmission coefficients are evaluated as in Eq. 3 with the summation over multipoles instead of spins and parities.

Since the total s-wave radiation width is generally measured only for stable isotopes plus a neutron, we have developed a systematic approach for estimating this value for the many unstable nuclei in our region of interest. Systematic descriptions of the average total s-wave radiation width generally exhibit a dependence on the mass and s-wave resonance spacing $\left(D_{0}\right)$ of the nucleus (Gardner 1975). We find that the measured radi- 

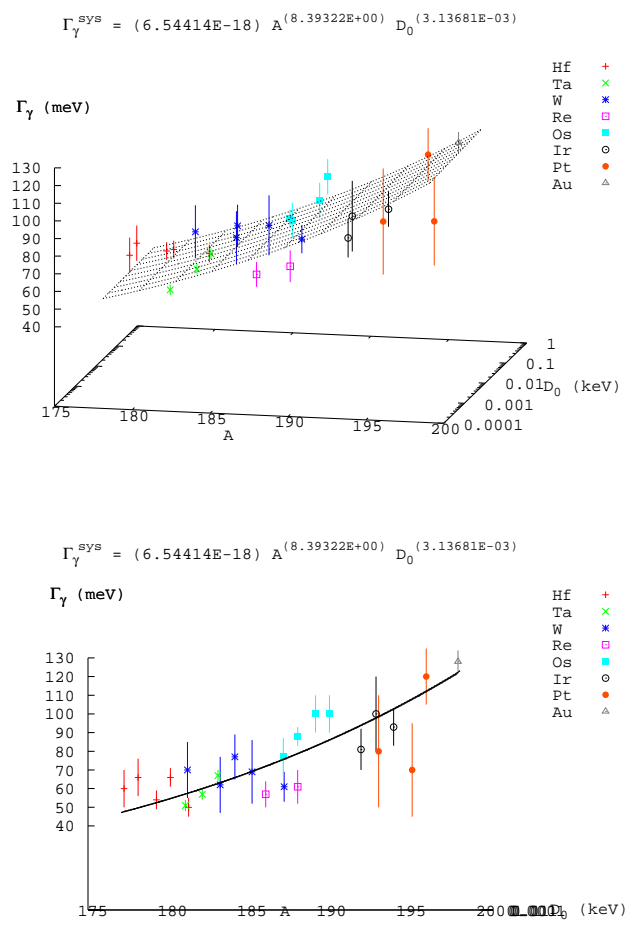

Fig. 7.- Systematics for average total s-wave radiation widths. The systematic is presented from two perspectives. The upper pane shows the relative mass and resonance spacing of the nuclei with measured radiation widths. The lower pane show the same systematic, only looking directly down the systematic plane to illustrate deviations of the systematic from measured values.

ation widths from (Belgya et al. 2005) are generally well fit by a parameterized curve in the $(A$, $D_{0}$ ) coordinate space:

$$
\begin{aligned}
\left\langle\Gamma_{\gamma}\right\rangle_{0}^{\text {sys }}= & 6.544 \times 10^{-18} A^{8.393} \\
& \times D_{0}^{0.00313}
\end{aligned}
$$

This systematic is shown in Figure (7). Whenever they are available, we use measured radiation widths instead of systematics to normalize the photon transmission coefficients.

When used to calculate neutron capture $(\mathrm{n}, \gamma)$ cross sections we found that the normalization to the photon-transmission function provided by the $\left\langle\Gamma_{\gamma}\right\rangle_{0}^{\text {sys }}$ systematic reproduced measured Maxwellian Averaged cross sections (MACS) to about a factor of 2 (Fig. 8). To improve on this we developed a secondary systematic to be applied as a multiplier to the primary systematic for

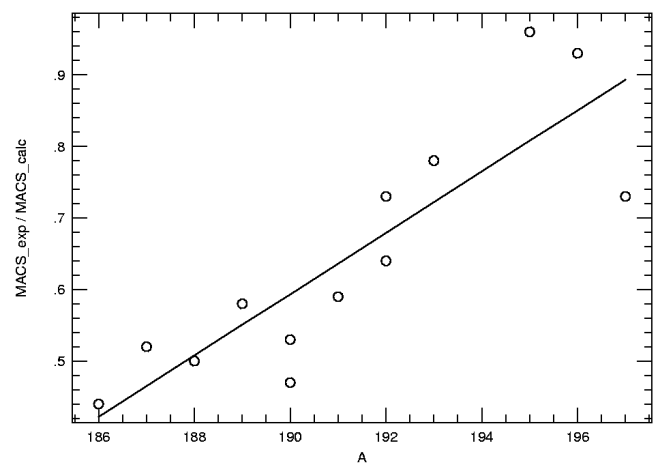

Fig. 8.- Systematic for the ratio of MACS (experimental to those calculated with the systematic in Fig. 7) to be applied as a multiple of the primary $\left\langle\Gamma_{\gamma}\right\rangle_{0}^{\text {sys }}$ systematic (Eq. 20). The fit is given in Eq. 21.

$\left\langle\Gamma_{\gamma}\right\rangle_{0}^{\text {sys }}$ (Eq. 20) that is a linear fit to the ratio of the experimental MACS to the calculated MACS at $30 \mathrm{keV}$ in the range $186 \leq A \leq 200$ (Fig. 8, Eq. 21). For compound nuclei with a measured MACS we apply the exact ratio. This systematic reproduces measured MACS to within $20 \%$ (often better). Comparison of both systematics is deferred to $\S$ 4.1.2.

$$
f_{\gamma \gamma}(A)=-7.541+0.0043 \times A
$$

\subsection{Nuclear Level Densities}

\subsubsection{Level Density Models}

Another important input to the statistical model code, especially for the capture reactions, is the nuclear level density. For this project, we have adopted a standardized, semi-empirical approach (Gilbert \& Cameron 1965) which is numerically efficient, can be tied to experimental data, and is fairly accurate. The level density is described by two functions. Both are energy dependent, the second factor contains the spin dependence. This is the "Back-shifted Fermi Gas" formulation of the nuclear level density:

$$
\rho(U, J)=\rho(U) f(U, J)
$$

where $\rho(U)$ is the state density, with $U=E-\Delta$ the back-shifted energy. $\Delta$ is the so called "pairing energy", and $J$ is the spin of the compound nucleus. We will further treat each of these in two ways, depending on the excitation energy of 
interest. The demarcation point will be roughly between the energy range of the known excited levels of a given compound nucleus (the low energy domain), and near (and above) the neutron binding energy (the high energy domain).

For the high energy domain, we describe the level density assuming a Fermi gas formula,

$$
\begin{array}{r}
\rho(U)=\frac{\sqrt{\pi}}{12} \frac{\exp (2 \sqrt{a U})}{a^{1 / 4} U^{5 / 4}} \frac{1}{\sqrt{2 \pi} \sigma} \\
f(U, J)=\frac{2 J+1}{2 \sigma^{2}} \exp \left[\frac{-\left(J+\frac{1}{2}\right)^{2}}{2 \sigma^{2}}\right]
\end{array}
$$

where $a(E)$ is the level density parameter (in $\left.\mathrm{MeV}^{-1}\right)$. The spin cutoff parameter $\sigma^{2}$ is defined as

$$
\sigma^{2}=\lambda \sqrt{a U} A^{2 / 3}
$$

The level density assumes an equal distribution of parity states. Note that at low excitation energy (for a positive back-shift), Eq. 23 diverges. At low energies, the nuclear level density is better described by a constant temperature formula:

$$
\rho(E) \propto \exp \frac{E-E_{0}}{T}
$$

The level density parameters can be calculated using experimental data. For the Fermi-gas state density (Eq. 23), the level density parameter, $a(E)$, can be related to the average level spacing $\left(D_{0}\right)$ near the neutron binding energy. The pairing energies used in the calculation of the back shifted energy are calculated as differences of binding energies (Bohr \& Mottelson). The constant temperature parameters $E_{0}$ and $T$, can be chosen to provide a state density that goes through the low lying spectroscopic levels subject to the choice of a matching energy, $E_{x}$, chosen someplace between the high and low energy regions of interest, at which the two state densities match (point and slope). We describe below how we determined these parameters for all of the nuclei considered in this study.

\subsubsection{Level Densities Above the Neutron Bind- ing Energy}

Our goal is to fit the level density parameter $a$ in Eq. 23 to experimental data where available. We adopt an energy dependent form, $a(U, Z, N)$, (Iljinov et al. 1992), and begin by fixing the spin cutoff parameter and the pairing energies.

\section{The Spin Cutoff Parameter}

The spin cutoff parameter $\sigma^{2}$, Eq. 25, characterizes the spin distribution of the Fermi gas level density. It depends on the parameters $a$, the level density parameter, and $\lambda$, which determines the effective moment of inertia for the nucleus in question. In principle it could be determined by experiment, for example, by comparing ratios of cross sections leading to different isomers of the product nucleus (Keisch 1963). Because data like this is often sparse, especially in the limited regions of the periodic chart we are interested in, and because we are often interested in reactions that proceed on or through radioactive species where no such data exists, we must resort to models. In our analysis, we fix $\lambda=1$ in Eq. 25, corresponding to the moment of inertia of a rigid sphere.

\section{Pairing Energies}

In determining the back-shift $\Delta$, also known as the pairing energy, we used a slightly modified version of the method of Rauscher (Rauscher et al. 1997). The total pairing energy is equal to the sum of the proton and neutron pairing energies

$$
\begin{aligned}
\Delta(Z, N) & =\frac{1}{2}\left(\Delta_{p}+\Delta_{n}\right) \\
\Delta_{p}(Z, N) & =E^{G}(Z, N) \\
& -\frac{1}{2} E^{G}(Z-1, N) \\
& -\frac{1}{2} E^{G}(Z+1, N) \\
\Delta_{n}(Z, N) & =E^{G}(Z, N) \\
& -\frac{1}{2} E^{G}(Z, N-1) \\
& -\frac{1}{2} E^{G}(Z, N+1)
\end{aligned}
$$

where $E^{G}(Z, N)$ is the binding energy of the nucleus $(Z, N)$. In calculating the binding energies of the various nuclei, we use the experimental mass excesses listed in the Möller and Nix tables (Möller et al. 1995).

\section{The Level Density Parameter}

Given $\Delta$ and $\sigma^{2}$, the level density parameter $a(U)$ can be related to the average "s"-wave level spacing at the neutron binding energy $\left(D_{0}\right)$ where such quantities are measured. In particular we assume an energy dependent level density parameter

$$
a(U)=\tilde{a}\left[1+\delta W \frac{f(U)}{U}\right]
$$




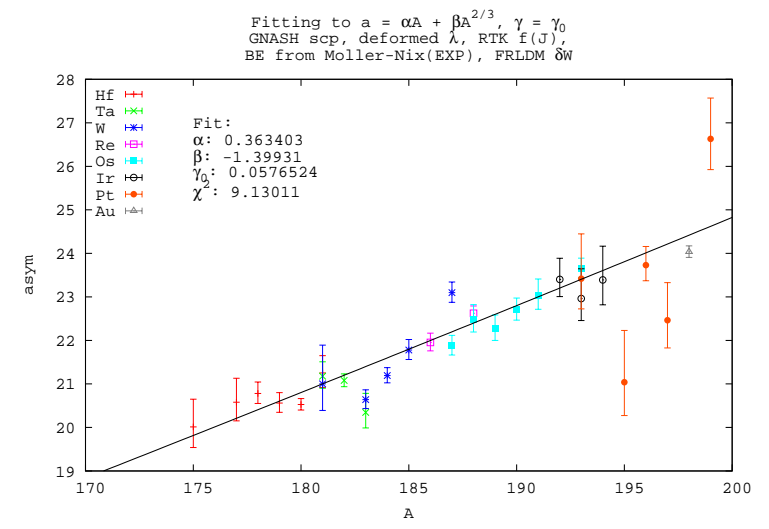

Fig. 9.- Three parameter fit to derived "experimental" asymptotic level density parameters, used to systematically determine unknown $\tilde{a}$. The data are obtained from measured s-wave resonance spacing's listed in (Belgya et al. 2005), assuming our chosen parameterizations for the shell correction, backshift and spin cutoff parameter. Our systematic, fit only to the data shown in this figure, is represented by the solid black line.

with $f(U)=1-\exp (-\gamma U)$ (Iljinov et al. 1992). We further assume that $\tilde{a}$ is of the form $\tilde{a}=$ $\alpha A+\beta A^{2 / 3}$, similar to (Rauscher et al. 1997). We adopt the so-called "microscopic correction" from (Möller et al. 1995) as our shell corrections, similar to (Rauscher et al. 1997), i.e. $\delta W=E_{m i c .}$.

Using the relation

$$
D_{\text {calc }}=\frac{2}{\rho\left(U, J=\frac{1}{2}\right)}
$$

for nuclei with spin $s=0$ and

$$
D_{\text {calc }}=\frac{2}{\rho\left(U, J=s+\frac{1}{2}\right)+\rho\left(U, J=s-\frac{1}{2}\right)}
$$

for nuclei with $s \neq 0$, we numerically solve for the values of $\alpha, \beta$, and $\gamma$ that minimize the quantity

$$
\chi^{2}=\sum_{i}\left(\frac{D_{0}^{\text {calc }}-D_{0}^{\mathrm{exp}}}{\delta D_{0}^{\exp }}\right)^{2}
$$

where $\delta D_{0}^{\exp }$ is the error in the measured $D_{0}$ and the sum is taken over measured $D_{0}$ for target nuclei in the range $72 \leq Z \leq 79,174 \leq A \leq 198$. The resulting fit finds $\alpha=0.363 \pm 0.002, \beta=$ $-1.399 \pm 0.068$, and $\gamma=0.0577 \pm 6.18 \times 10^{-6}$, and is shown in Figure 9.

\subsubsection{Level Densities Below the Neutron Bind- ing Energy}

For the lower energy regions, below the neutron binding energy $B_{n}$, the nuclear level density has the same formulation as Eq. 22. However, particularly at and below the pairing energy $\Delta$, the state density in Eq. 23 becomes imaginary. Unfortunately, experimental level schemes are rarely known above $2 \mathrm{MeV}$ of excitation energy. In practice we are forced again to assume a model and use all available experimental data to constrain its parameters.

Of course the two prescriptions for the level density must match at some energy intermediate to where they are constrained by experiment. Henceforth we will refer to the high energy level density as $\rho_{1}$, and the low energy density as $\rho_{2}$.

Gilbert and Cameron (Gilbert \& Cameron 1965) noticed that the cumulative number of observed levels (the so-called staircase plot, which increase exponentially), can be fit with straight lines in a semi-log plot. They adopted a constant temperature formula to fit these:

$$
N(E)=\exp \left[\frac{E-E_{0}}{T}\right]
$$

with $N(E)$ being the cumulative number of levels at excitation energy $E, E_{0}$ and $T$ are two free parameters to be fit to the observed level structure. The observable level density is given by

$$
\rho_{1}(E)=\frac{d N(E)}{d E}=\frac{1}{T} \exp \left[\frac{E-E_{0}}{T}\right]
$$

From classical thermodynamics, we have a definition of the nuclear temperature

$$
\frac{d}{d E} \log \rho_{1}(E)=\frac{1}{T}
$$

where $T$ now takes on the meaning of a nuclear temperature which is constant in the region of the discrete levels. We assume that Eq. 32 can be extrapolated from the region of the known discrete levels to higher energies, where the Fermi-gas level density $\left(\rho_{1}\right)$ is valid. We then define the notion of a fit to the total level density over the entire range as being achieved if: a) a good fit can be made to the low lying levels, b) the observed level spacing at the neutron binding energy is exactly reproduced, and c) the energy of the matching point $E_{x}$ for the two prescriptions falls between $E=0$ and $E=$ $B_{n}$, and that they match at this point with the same slope, i.e. for $E=E_{x}$ :

$$
\rho_{1}\left(E_{x}\right)=\rho_{2}\left(E_{x}\right)
$$




$$
\frac{d \log \rho_{1}\left(E_{x}\right)}{d E}=\frac{d \log \rho_{2}\left(E_{x}\right)}{d E}
$$

From the first of these, we can determine $E_{0}$ :

$$
E_{0}=E_{x}-T \log T \rho_{2}\left(U_{x}\right)
$$

where $U_{x}=E_{x}-\Delta$. The second condition can be satisfied by assuming that at $E_{x}$ the constant nuclear temperature $T$ of the low lying states is equal to the energy dependent nuclear temperature $\tau\left(U_{x}\right)$ of the high excited states,

$$
\begin{aligned}
\frac{1}{T} & =\sqrt{\frac{a}{U_{x}}}-\frac{3}{2 U_{x}} \\
& +\frac{(\tilde{a}-a)\left(1+\gamma U_{x}\right)+\tilde{a} \gamma \delta W}{\sqrt{a U_{x}}}
\end{aligned}
$$

where $a$ is given by Eq. 28. If there is no shell correction, the latter term in the above equation is zero. Typical values for the matching energy are $2 \leq E_{x} \leq 8 \mathrm{MeV}$, and are approximated by $E_{x}=2.5+\frac{150}{A}+\Delta$ (Gilbert \& Cameron 1965). The constant temperature fit to the low lying levels of ${ }^{198} \mathrm{Au}$ which is typical of the fits for all nuclei in this region is presented in Figure 10.

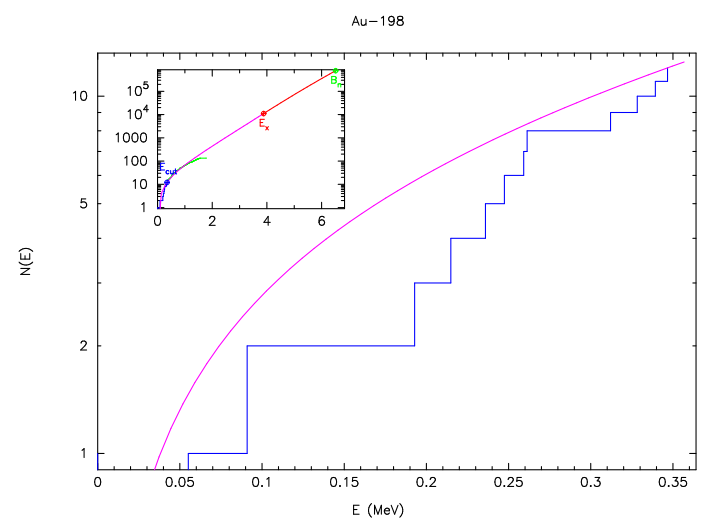

Fig. 10.- Constant temperature level density fits to the low lying spectroscopic levels of ${ }^{198} \mathrm{Au}$.

\section{Behavior of the Spin Cutoff Parameter Below $E_{x}$}

At the matching energy $E_{x}$, the spin cutoff parameter is given by Eq. 25. One may define $E_{\text {cut }}$ as the energy of the highest known excited level for which energy, spin and parity are explicitly known. We define the following for the behavior of the spin cutoff parameter:

$$
\begin{aligned}
\sigma_{H}^{2} & =\sigma_{E_{x}}^{2} \\
U_{L} & =\max \left(E_{\text {cut }}-\Delta, 0.1\right)
\end{aligned}
$$

$$
\begin{aligned}
\sigma_{L}^{2} & =\lambda \sqrt{a U_{L}} A^{2 / 3} \\
\sigma_{G}^{2} & =\sigma_{L}^{2}+\frac{E-\frac{1}{2} E_{c u t}}{E_{x}-\frac{1}{2} E_{c u t}}\left(\sigma_{H}^{2}-\sigma_{L}^{2}\right)
\end{aligned}
$$

The form $\sigma_{G}^{2}$ is then used between $\frac{1}{2} E_{c u t}$ and $E_{x}$. This is the GNASH prescription (Chadwick 1998).

The behavior of $\sigma^{2}$ below $E_{x}$ will only affect the level density used in Hauser-Feshbach calculations between $E_{c u t}$ and $E_{x}$, since the discrete levels are accounted for individually. The changes that arise between $E_{c u t}$ and $E_{x}$ are generally small.

The fitted parameters for the total level density are presented in Table A.4. The symbols in the legend are the same as described above. In column five, a "*" indicates the asymptotic level density parameter $\tilde{a}$ was derived from an experimentally known level spacing $D_{0}$, otherwise it was derived from the systematic shown in Figure 9.

\subsection{Considerations Regarding the Exci- ton Model}

When including alpha particles as a possible exit channel, one must account for them in the pre-equilibrium phase of the reaction. In particular, they must be accounted for in the exciton model. Generally, the description of alpha particle emission in the pre-equilibrium model is a straightforward extension of the description of neutron or proton emission, given the tendency of nucleons to pre-form alpha clusters in the nucleus. In making such an extension, one introduces a parameter $\phi$ which represents the probability that the incoming particle will strike a pre-formed alpha cluster (Milazzo-Colli \& Braga-Marcazzan 1973). It follows that the larger values of $\phi$ will result in a higher probability of subsequent alpha emission, thus enhancing the $(\mathrm{n}, \alpha)$ reactions.

In our calculations, we have chosen a value of $\phi=0.20$, although previous considerations of alpha emission suggest that this value may fall anywhere in the range of $0.1 \leq \phi \leq 0.8$ within the mass range of interest. We have used our chosen value primarily because it results in $(\mathrm{n}, \alpha)$ cross sections which best fit the available experimental data.

Since the alpha particle emission accounts for only a very small portion of the total reaction cross section (generally less than $1 \%$ ), variations in the $\phi$ parameter will only have minimal, if not negligible, effects on the other cross sections. 


\section{Modeled Cross Sections}

\subsection{Comparison to Measured Cross Sec- tions}

Having developed the various input quantities based on available experimental data in the previous section, we now turn to the results of the STAPRE-H95 model and compare to available measured cross sections in the region of interest. We restrict our attention primarily to ground state targets of ${ }^{191} \mathrm{Ir},{ }^{193} \mathrm{Ir}$, and ${ }^{197} \mathrm{Au}$ (stable loaded detector elements closest in mass to the measured radioactivities). Comparisons to other measured cross sections are provided in the appendices.

\subsubsection{Comparison to experimental $(n, \gamma)$ capture cross sections}

In Figure 11 we present comparisons for neutron capture reactions on select targets. Shown is the activation cross section (solid black lines in all plots that follow) defined as the sum of emission (both particle emission and gamma-ray cascade) from the compound nucleus that eventually leads to the ground state of the product (final) nucleus. We also provide (where appropriate) separate cross sections that decay to the ground state (red lines), and any long lived isomers (blue and green lines). See Appendix A for a list of the isomers and their respective half-lives. These cross sections are plotted against the available experimental data, taken from the Experimental Nuclear Reaction Data File (EXFOR 2006). Cross sections for the total, ground, and isomeric states are colored in a similar manner to the modeled cross sections (grey is activation, orange is to ground, and light blue and green to an isomer, respectively), with different symbols distinguishing results from various experiments.

Our results are in overall good agreement with the wealth of measured data near $100 \mathrm{keV}$ for ${ }^{191} \operatorname{Ir}(\mathrm{n}, \gamma){ }^{192} \operatorname{Ir}$ and ${ }^{193} \operatorname{Ir}(\mathrm{n}, \gamma){ }^{194} \operatorname{Ir}$. The only general exception is around $14 \mathrm{MeV}$ incident energy, where direct capture mechanisms (not included in our calculations) begin to become significant. Since the neutron capture cross section is usually two or three orders of magnitude smaller than the dominant neutron induced reactions at these energies, neglecting direct capture will introduce negligible errors into any network calculations. For ${ }^{191,193} \operatorname{Ir}(\mathrm{n}, \gamma)^{192,194} \operatorname{Ir}$, our calculation is high by roughly a factor of 1.5 between $\sim 0.5-1.0 \mathrm{MeV}$. Below $5 \mathrm{keV}$, the effects of individual resonances (which cannot be reproduced with the statistical
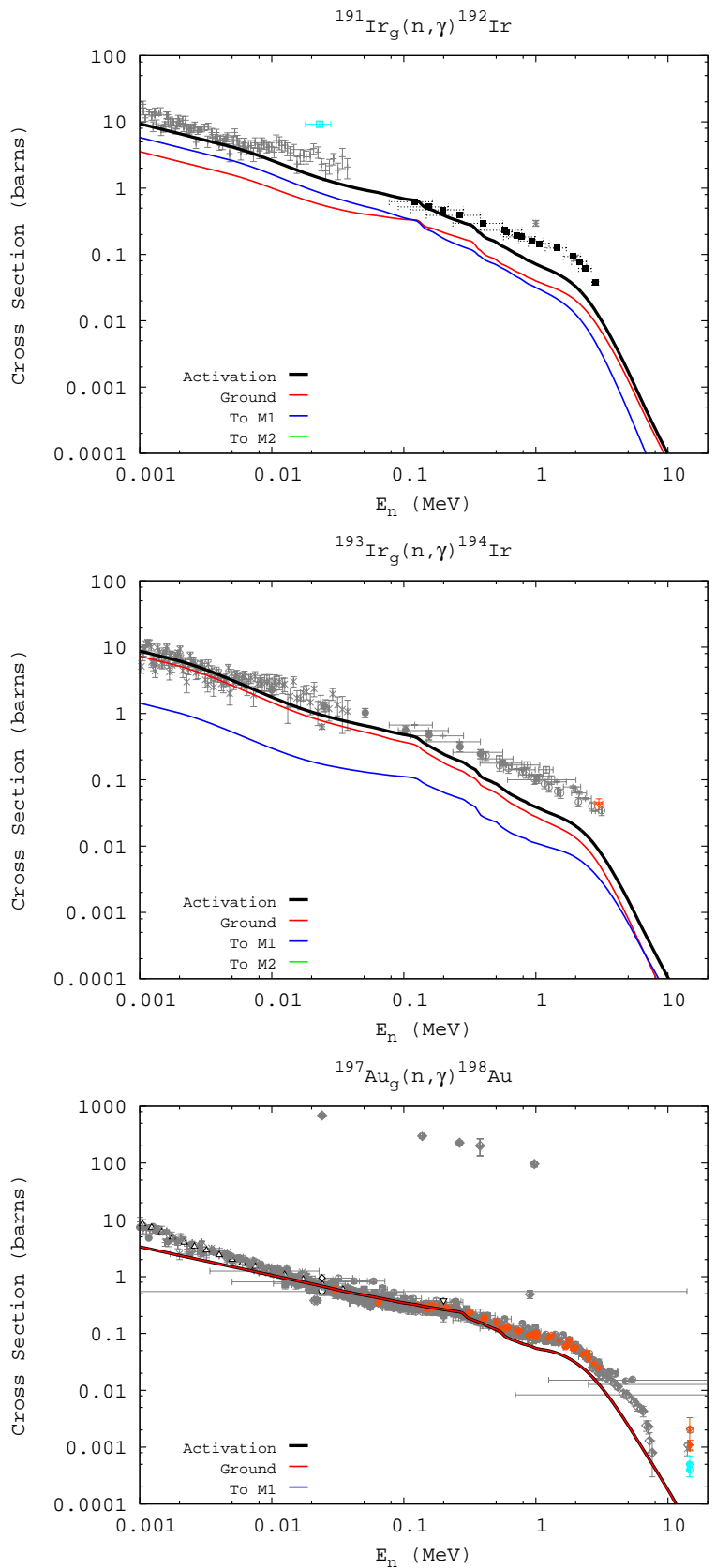

Fig. 11. - Calculated vs. measured $(\mathrm{n}, \gamma)$ cross sections on select stable isotopes in the region of interest. The data is taken from (EXFOR 2006). The black, red, and blue solid lines represent our modeled cross sections (total, leading to the ground state, and leading to the first isomer, respectively). The grey, orange, and light blue data points are measured cross section data (total, ground state, and first isomer).

model) are present. For ${ }^{197} \mathrm{Au}(\mathrm{n}, \gamma){ }^{198} \mathrm{Au}$ our calculation is in excellent agreement with the data 
Table 1: Comparison of our modeled $(\mathrm{n}, \gamma)$ cross sections to experimental data at $30 \pm 2 \mathrm{keV}$

\begin{tabular}{cllllll}
\hline \hline${ }^{A} Z$ & Res. & $\mathrm{N}$ & $\bar{\sigma}$ & Dev. & $\sigma_{\text {mod. }}$ & $\bar{\sigma} / \sigma_{\text {mod. }}$ \\
\hline${ }^{187} \mathrm{Os}$ & $\mathrm{A}$ & 3 & 0.870 & 0.035 & 0.689 & 1.262 \\
${ }^{190} \mathrm{Os}$ & $\mathrm{A}$ & 1 & 0.477 & 0.000 & 0.262 & 1.818 \\
${ }^{192} \mathrm{Os}$ & $\mathrm{A}$ & 2 & 0.179 & 0.072 & 0.259 & 0.690 \\
${ }^{191} \mathrm{Ir}$ & $\mathrm{A}$ & 2 & 2.310 & 0.460 & 1.215 & 1.902 \\
${ }^{193} \mathrm{Ir}$ & $\mathrm{A}$ & 2 & 1.671 & 0.310 & 0.838 & 1.994 \\
${ }^{196} \mathrm{Pt}$ & $\mathrm{G}$ & 1 & 0.062 & 0.000 & 0.171 & 0.363 \\
${ }^{198} \mathrm{Pt}$ & $\mathrm{A}$ & 2 & 0.251 & 0.010 & 0.111 & 2.270 \\
${ }^{197} \mathrm{Au}$ & $\mathrm{A}$ & 64 & 0.603 & 0.096 & 0.612 & 0.986 \\
\hline \multicolumn{6}{c}{ Average error: } & $55.0 \%$ \\
Average error (activation only): & $37.8 \%$ \\
\hline \multicolumn{6}{c}{}
\end{tabular}

over nearly the entire range available.

Additional comparisons for neutron capture cross sections are presented in Appendix B.1 where a similar degree of agreement between our calculations and experiment is found. Such results are considered good for $(\mathrm{n}, \gamma)$ activation cross sections. Using global systematics for the average radiation widths, $(\mathrm{n}, \gamma)$ cross sections can typically be modeled within a factor of two, sometimes to within 30\% (Hoffman et al. 1999).

A quantitative comparison of our calculated cross sections to the experimental data shown in Figures 11 and 30 is given in Table 1. For $(n, \gamma)$ reactions we restrict our analysis to data with incident neutron energies of $30 \pm 2 \mathrm{keV}$. For each target listed in column (1), the subsequent column entries identify: (2) Res., the state of the residual (product) nucleus (activation, ground state, isomer); (3) $\mathrm{N}$, the number of experimental data points falling within the incident energy range; (4) $\bar{\sigma}$, the weighted average of the measured data (in barns), with weights corresponding to the inverse geometric mean of the errors in cross section and energy (i.e. $\left.w_{i}=\left(d E^{2}+d \sigma^{2}\right)^{-1 / 2}\right)$; (5) Dev., the standard deviation from the mean (also in barns), which gives an indication of the spread in the experimental data; (6) $\sigma_{\text {mod. }}$, our modeled capture cross section (in barns); and (7) $\bar{\sigma} / \sigma_{\text {mod. }}$, the ratio of the weighted average of the data to our modeled value, which may be used as a scaling factor to be applied to a given cross section to bring it into conformity with its respective average experimental value. Also provided at the bottom of the table is an average absolute percent error (defined as $\left.\% E=N^{-1} \sum_{i=1}^{N}\left(\left|\sigma_{i}^{\text {mod. }}-\bar{\sigma}_{i}\right| / \bar{\sigma}_{i}\right) \times 100 \%\right)$ for all capture cross sections and the activation cross sections only.
Table 2: Comparison of our modeled Maxwellianaveraged (n, $\gamma$ ) cross sections (in millibarns) to recommended values at $30 \mathrm{keV}$ using the $\left\langle\Gamma_{\gamma}\right\rangle_{0}^{\text {sys }}$ systematic (1) and the product of this and the ratio of maxwellians systematic $f_{\gamma \gamma}(2)$

\begin{tabular}{cccc}
\hline \hline${ }^{A} Z$ & Rec. & Mod.(1) & Mod.(2) \\
\hline${ }^{186} \mathrm{Os}$ & $422 \pm 16$ & 964 & 348 \\
${ }^{187} \mathrm{Os}$ & $896 \pm 30$ & 1717 & 703 \\
${ }^{188} \mathrm{Os}$ & $399 \pm 15$ & 793 & 351 \\
${ }^{189} \mathrm{Os}$ & $1168 \pm 47$ & 2005 & 997 \\
${ }^{190} \mathrm{Os}$ & $295 \pm 45$ & 560 & 287 \\
${ }^{192} \mathrm{Os}$ & $311 \pm 45$ & 428 & 284 \\
${ }^{191} \mathrm{Ir}$ & $1350 \pm 43$ & 2283 & 1298 \\
${ }^{193} \mathrm{Ir}$ & $994 \pm 70$ & 1271 & 906 \\
${ }^{190} \mathrm{Pt}$ & $677 \pm 183$ & 1447 & 766 \\
${ }^{192} \mathrm{Pt}$ & $590 \pm 120$ & 928 & 599 \\
${ }^{195} \mathrm{Pt}$ & $860 \pm 200$ & 893 & 792 \\
${ }^{196} \mathrm{Pt}$ & $197 \pm 23$ & 211 & 186 \\
${ }^{197} \mathrm{Au}$ & $582 \pm 9$ & 797 & 625 \\
\hline \multicolumn{2}{c}{ Avg. \% error: } & (R1) $35.3 \%$ & (R2) $9.5 \%$ \\
\hline
\end{tabular}

On average, our modeled cross sections are within $55 \%$ of the weighted mean at $30 \mathrm{keV}$, although the large deviations in the data for ${ }^{191,193} \mathrm{Ir}$ are to be considered (only 2 values are available) and the large discrepancies for ${ }^{196,198} \mathrm{Pt}$ reflect the behavior of the 2nd systematic in a region where the data is declining. Our activation cross sections are within $38 \%$ on average.

\subsubsection{Comparison to Maxwellian averaged $(n, \gamma)$ capture cross sections}

Another comparison to experimental data comes from the extensive efforts to measure and evaluate Maxwellian averaged capture cross sections for astrophysical applications (Bao et al. 2000). The Maxwellian-averaged neutron capture cross section is defined as the reaction rate $\langle\sigma v\rangle$ divided by the mean velocity $v_{T}=\sqrt{2 k T / \mu}$ at a given temperature $T$. Here, $\mu$ is the reduced mass. For particle fluences and temperatures typical to stellar nucleosynthesis, the velocity distribution of the neutrons is well described by a Maxwell-Boltzmann distribution. In this case, the Maxwellian-averaged cross section reduces to (Beer et al. 1992)

$$
\begin{aligned}
\frac{\langle\sigma v\rangle}{v_{T}} & =\frac{\int_{0}^{\infty} \sigma_{n \gamma} v \Phi(v) d v}{v_{T}} \\
& =\frac{2}{\sqrt{\pi}(k T)^{2}} \int_{0}^{\infty} \sigma_{n \gamma}(E) W(E, k T) d E
\end{aligned}
$$



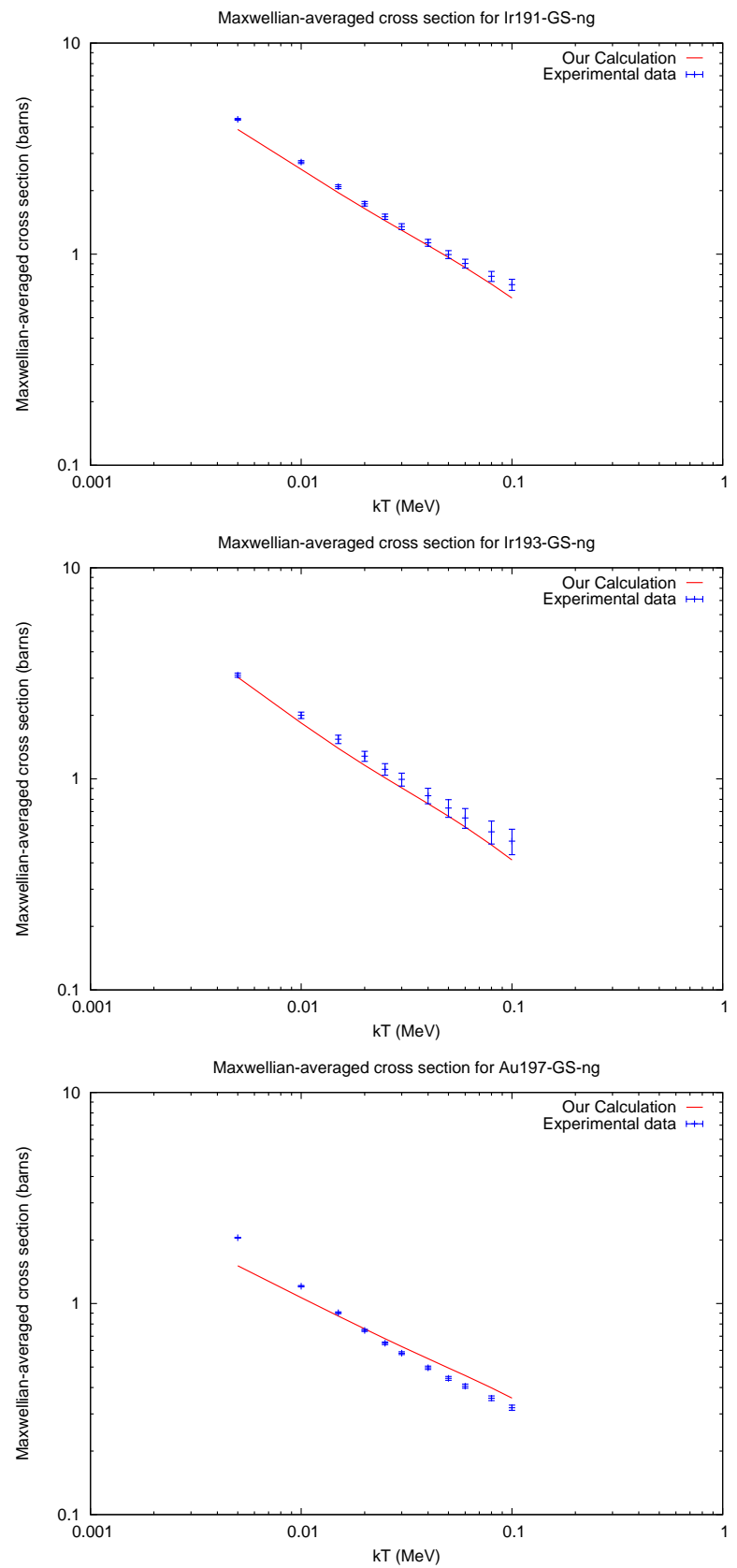

Fig. 12. - Calculated vs. recommended Maxwellian-averaged capture cross sections on ${ }^{191,193} \mathrm{Ir}$ and ${ }^{197} \mathrm{Au}$ stable targets. The data is taken from (Bao et al. 2000). The solid line represent our modeled cross section. The data points are the recommended values, with the error bars for each energy identical to the quoted error at 30 $\mathrm{keV}$.

where $W(E, k T)=E \exp (-E / k T)$ and $E$ is the center of mass energy.

Figure 12 compares our calculated Maxwellian- averaged capture cross sections for ${ }^{191,193}$ Ir targets to their measured counterparts (Bao et al. 2000). The error bars on all points are identical and represent the measured error for a given cross section at $30 \mathrm{keV}$. We used spline interpolation to determine the value of the $(n, \gamma)$ cross section between points on the energy grid. For energies below our lowest grid energy, we assume an $(n, \gamma)$ cross section with an $E_{l a b}^{-1 / 2}$ dependence. For energies greater than our highest grid energy, we take the cross section to be zero.

The ratios of recommended $30 \mathrm{keV}$ Maxwellianaveraged cross sections, relative to the modeled values, are listed in Table 2, giving the target, recommended cross section (with error, in millibarns), our modeled value (in mb), and the ratio of recommended to modeled cross sections using both the $\left\langle\Gamma_{\gamma}\right\rangle_{0}^{\text {sys }}$ systematic (Eq. 20) and the product of this and the $f_{\gamma \gamma}$ systematic (21), for which we obtain much better results, typically within $10 \%$. Additional figures for comparison are found in Appendix B.2.

\subsubsection{Comparison to experimental (n,n') cross sections}

In order to properly account for the population of the isomers via scattering we include $\left(n, n^{\prime}\right)$ reactions that couple all isomer excited states specified in table A. Our calculations agree very well with with the measured (n,n') data from ground state to first isomer in Figure 13. These are the only data sets available for the $\mathrm{Ir}$ and $\mathrm{Au}$ isotopes in this study, although our success with other isotopes of $\mathrm{Os}, \mathrm{Pt}$, and $\mathrm{Hg}$ are similar (but not shown).

\subsubsection{Comparison to experimental (n,2n) cross sections}

Our calculated $(n, 2 n)$ cross sections for select targets are presented in Figure 14 and exhibit very good agreement against measured data for all three targets considered including cross sections to the various final states. Although the loaded Ir isotopes have limited (one or two) experiments reporting activation and ground final state cross sections, each has multiple measurements of the first isomer. The gold isotope is of course a laboratory standard. The dispersion in the measured data is larger but the various data sets are generally consistent with each other.

Appendix B.3 presents additional comparisons between our modeled $(\mathrm{n}, 2 \mathrm{n})$ cross sections and experiment. Most of the data applies to measure- 

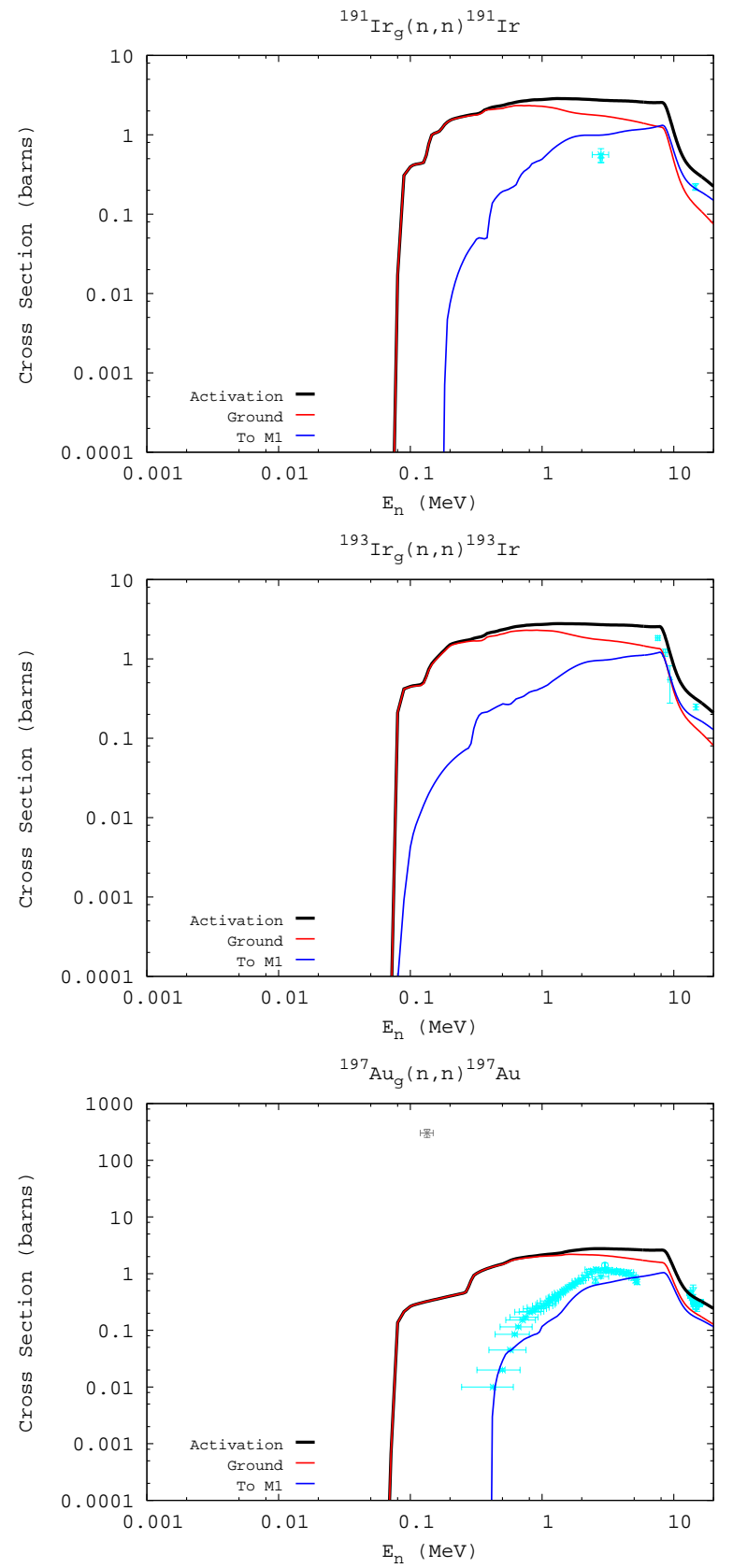

Fig. 13.- Modeled (n,n') cross sections compared to measurement. The data is taken from (EXFOR 2006). The black, red, and blue solid lines represent our modeled cross sections (total, leading to the ground state, and leading to the first isomer, respectively). The grey, orange, and light blue data points are measured cross section data (total, ground state, and first isomer).

ments of cross sections proceeding to ground and first isomer states, which are generally well reproduced by our calculations (see particularly ${ }^{184} \mathrm{Os}$,
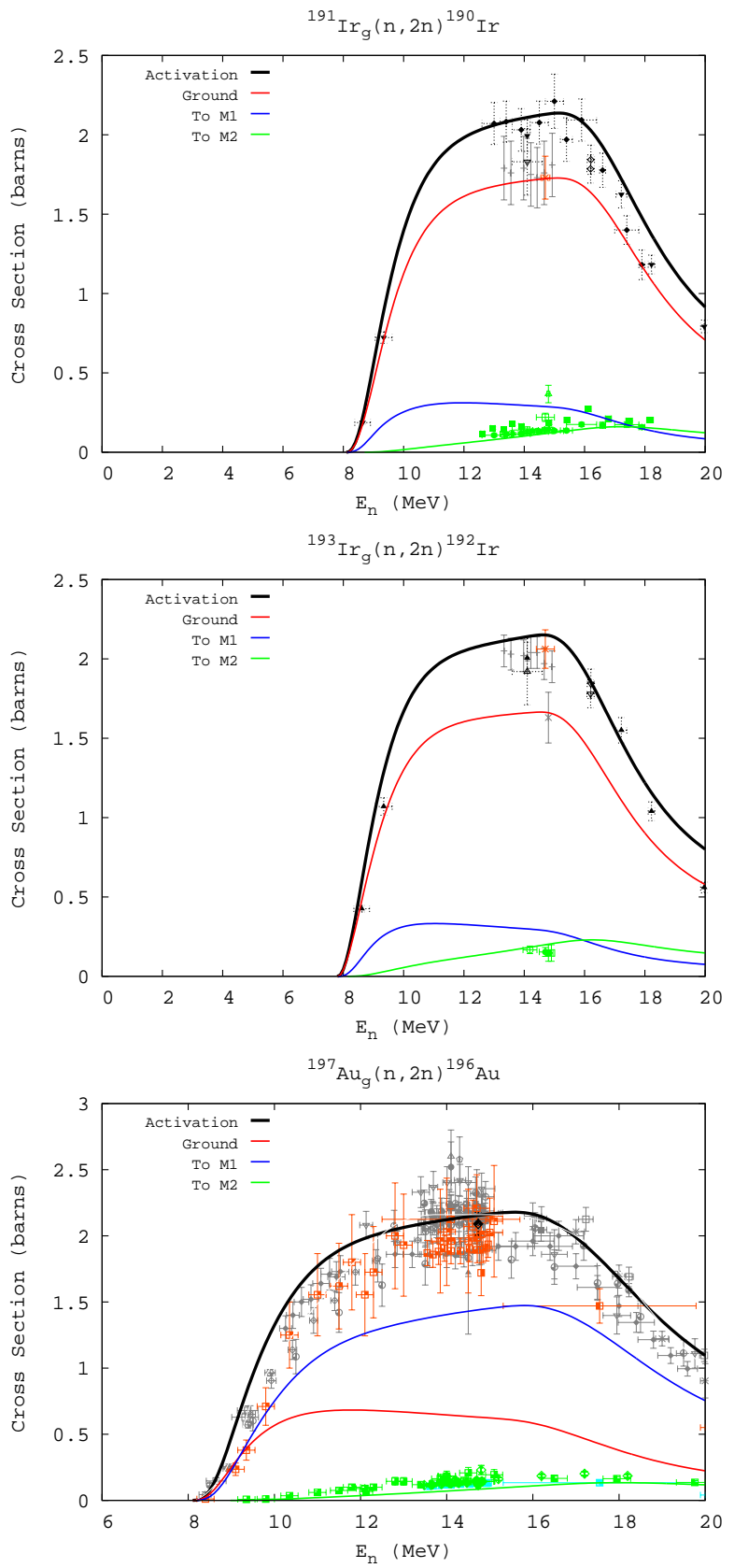

Fig. 14.- Calculated vs. measured $(\mathrm{n}, 2 \mathrm{n})$ cross sections on select stable isotopes in the region of interest. The data is taken from (EXFOR 2006). The black, red, and blue solid lines represent our modeled cross sections (total, leading to the ground state, and leading to the first isomer, respectively). The grey, orange, and light blue data points are measured cross section data (total, ground state, and first isomer).

${ }^{196} \mathrm{Hg}$ ). The data for activation measurements also agrees well over the limited range (near $14 \mathrm{MeV}$ ) 
Table 3: Comparison of our modeled $(\mathrm{n}, 2 \mathrm{n})$ cross sections to experimental data at $14.7 \pm 0.1 \mathrm{MeV}$ for activation (A), ground state (GS) and isomer (M1) targets.

\begin{tabular}{lllllll}
\hline \hline${ }^{A} Z$ & Res. & $\mathrm{N}$ & $\bar{\sigma}$ & Dev. & $\sigma_{\text {mod. }}$ & $\bar{\sigma} / \sigma_{\text {mod }}$ \\
\hline${ }^{186} \mathrm{Os}$ & A & 2 & 1.787 & 0.312 & 2.110 & 0.847 \\
${ }^{192} \mathrm{Os}$ & GS & 3 & 1.563 & 0.369 & 1.806 & 0.865 \\
${ }^{192} \mathrm{Os}$ & M1 & 5 & 0.899 & 0.140 & 0.392 & 2.295 \\
${ }^{192} \mathrm{Os}$ & A & 5 & 2.135 & 0.142 & 2.198 & 0.971 \\
${ }^{191} \mathrm{Ir}$ & GS & 1 & 1.730 & 0.000 & 1.723 & 1.004 \\
${ }^{191} \mathrm{Ir}$ & A & 1 & 1.760 & 0.000 & 2.129 & 0.827 \\
${ }^{193} \mathrm{Ir}$ & GS & 1 & 2.062 & 0.000 & 1.663 & 1.240 \\
${ }^{193} \mathrm{Ir}$ & A & 1 & 1.970 & 0.000 & 2.151 & 0.916 \\
${ }^{192} \mathrm{Pt}$ & A & 3 & 2.019 & 0.014 & 2.127 & 0.949 \\
${ }^{198} \mathrm{Pt}$ & GS & 2 & 1.020 & 0.056 & 2.347 & 0.435 \\
${ }^{198} \mathrm{Pt}$ & A & 4 & 1.842 & 0.095 & 2.347 & 0.785 \\
${ }^{197} \mathrm{Au}$ & GS & 9 & 2.001 & 0.105 & 2.160 & 0.926 \\
${ }^{197} \mathrm{Au}$ & A & 31 & 2.140 & 0.135 & 2.160 & 0.991 \\
\hline \multicolumn{6}{c}{ Average error: } & $23.9 \%$ \\
\multicolumn{5}{c}{ Average error (activation only): } & $11.8 \%$ \\
\hline
\end{tabular}

that it is often provided, the exceptions being ${ }^{192} \mathrm{Os}$ and ${ }^{204} \mathrm{Hg}$. For the cases where only ground and isomer data is provided, the sum closely represents the activation cross sections shown (to within $10 \%)$.

As a further means of evaluating our (n,2n) cross sections, we present in Table 3 a quantified comparison of our results to the experimental data shown in Figures 14 and 32. We include all data with incident energies of $14.7 \pm 0.1 \mathrm{MeV}$. The format of the table is identical to Table 1.

The average deviation (by percentage error) of our cross sections from the weighted means for the $(\mathrm{n}, 2 \mathrm{n})$ reactions when only activation measurements are considered is $11.8 \%$. For two cases (namely ${ }^{186} \mathrm{Os}(\mathrm{n}, 2 \mathrm{n}){ }^{185} \mathrm{Os}$, and ${ }^{198} \mathrm{Pt}(\mathrm{n}, 2 \mathrm{n}){ }^{197} \mathrm{Pt}$ ) there are several activation data sets that are not in agreement with each other. In each of these cases, our calculation lies close to at least one of the data sets. Excluding these two reactions, the average error for the $(\mathrm{n}, 2 \mathrm{n})$ activation cross sections is $9.5 \%$.

\subsubsection{Comparison to experimental $(n, 3 n)$ cross sections}

Our calculated $(\mathrm{n}, 3 \mathrm{n})$ cross sections for ${ }^{191} \mathrm{Ir}$ and ${ }^{197} \mathrm{Au}$ are shown in Figure 15. No experimental data is available for ${ }^{193} \mathrm{Ir}$. The thresholds for this reaction are above $14 \mathrm{MeV}$ in nearly all cases in this mass range, reflecting the fall off of
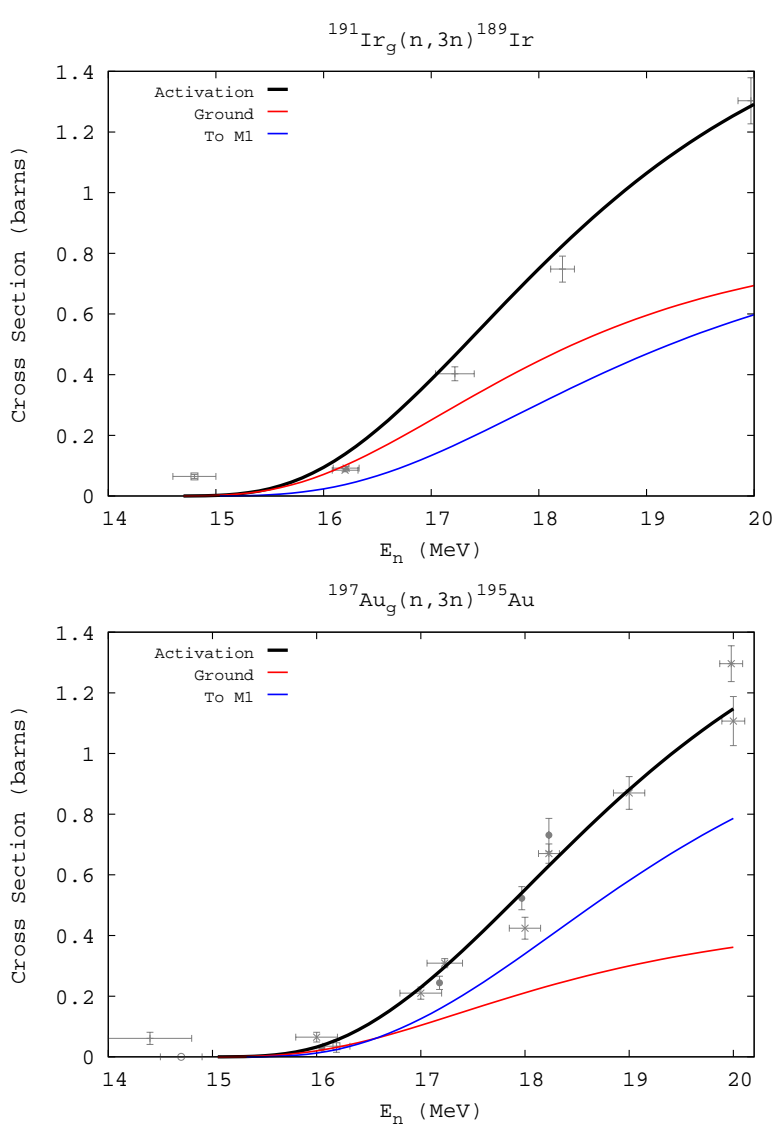

Fig. 15. - Calculated vs. measured (n,3n) cross sections on select stable isotopes in the region of interest. The data is taken from (EXFOR 2006). The black, red, and blue solid lines represent our modeled cross sections (total, leading to the ground state, and leading to the first isomer, respectively). The grey, orange, and light blue data points are measured cross section data (total, ground state, and first isomer).

the $(\mathrm{n}, 2 \mathrm{n})$ reaction channel. Our calculated cross sections are in excellent agreement over the entire range required, although only activation data is available. For our applications this is not a problem since the the UGT program measured the ground state activity for loaded ${ }^{191} \operatorname{Ir}(\mathrm{n}, 3 \mathrm{n}){ }^{189} \mathrm{Ir}$ and ${ }^{197} \mathrm{Au}(\mathrm{n}, 3 \mathrm{n}){ }^{195} \mathrm{Au}$.

\subsubsection{Comparison to experimental $(n, p)$ and $(n, \alpha)$ cross sections}

For most stable isotopes, the neutron capture and $(\mathrm{n}, 2 \mathrm{n})$ reactions are the dominant neutron induced reaction channels at low and high incident energies, respectively. However, as one moves to the proton rich side of stability, proton separa- 
tion energies become small, and reaction channels involving charged particles in the exit channel, specifically $(n, p),(n, n p)$, and $(n, \alpha)$ can become dominant.

In Figure 16 we present our modeled cross sections for select target isotopes compared to measurement. In all cases it appears our calculations over-predict the measured cross sections by roughly a factor of three. This behavior is a common feature in our (n,p) cross sections, and is likely due to the simple treatment of preequilibrium. Fortunately, the (n,p) cross section is rarely dominant above $\sim 12 \mathrm{MeV}$ (see Appendix $\mathrm{C})$, so that errors in our calculation at and above $14 \mathrm{MeV}$ will likely have insignificant impact in network calculations.

Another possibility is the parameterization for our proton optical model. Very little data was available to constrain this (there is some on ${ }^{197} \mathrm{Au}$ from a fission experiment at $190 \mathrm{MeV}$ ) so it could be off by up to a factor of three. To check this, we show again in Figure 16 the well measured cross section ${ }^{197} \mathrm{Au}(\mathrm{p}, \mathrm{n}){ }^{197} \mathrm{Hg}$. The activation, ground, and first isomer cross sections all peak near 10 $\mathrm{MeV}$, the difference for the activation cross section is a factor of 1.4. Of note is the behavior of all three cross sections above $14 \mathrm{MeV}$, the experiments indicate a rapid decline, whereas the calculations are more gradual. We attribute this to the pre-equilibrium normalization more than the proton optical model.

Additional comparisons of our (n,p) cross sections to experiment can be found in Appendix B.4. The agreement between the calculation and measurement in comparable to that presented in Figure 16.

\subsubsection{Comparison to experimental $(n, \alpha)$ cross sections}

The $(\mathrm{n}, \alpha)$ channel for these heavy systems is generally small compared to other open channels. The availability and quality of $(n, \alpha)$ data in this region is comparable to what is available for $(n, p)$ which precludes us from making a meaningful quantitative statistical analysis of our $(n, \alpha)$ calculations. However, we present in Figure 17 a sample of our results compared to data for the loaded detector elements of Ir and Au. Additional comparisons can be found in Appendix B.5.

One thing that is clear for both the (n,p) and $(\mathrm{n}, \alpha)$ cross sections is that they do not compete with the dominant $(n, 2 n)$ and $(n, g)$ channels. With cross sections only measured near $14 \mathrm{MeV}$,
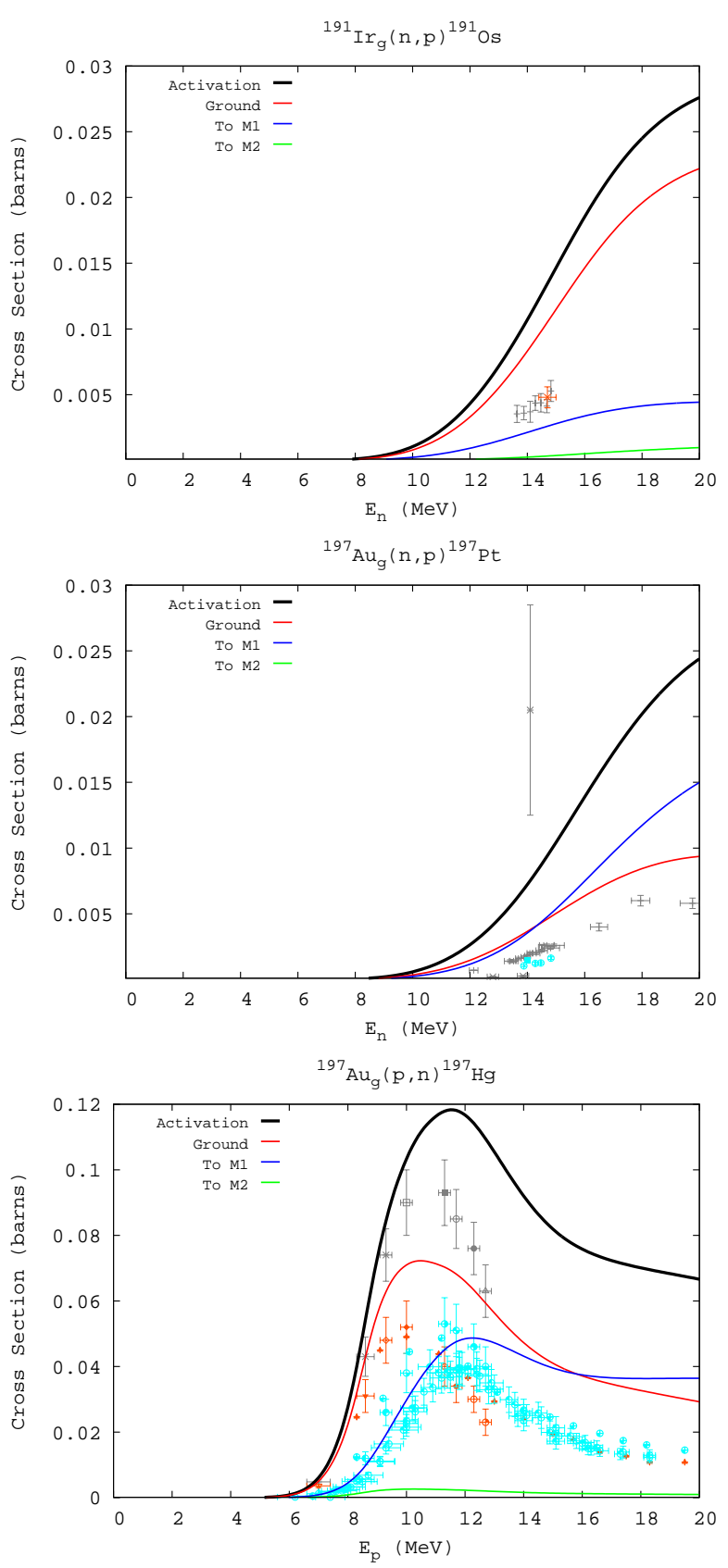

Fig. 16. - Calculated vs. measured (n,p) cross section on ${ }^{191} \mathrm{Ir}$ and the $(\mathrm{n}, \mathrm{p})$ and $(\mathrm{p}, \mathrm{n})$ cross sections on ${ }^{197} \mathrm{Au}$. The data is taken from (EXFOR 2006). The black, red, and blue solid lines represent our modeled cross sections (total, leading to the ground state, and leading to the first isomer, respectively). The grey, orange, and light blue data points are measured cross section data (total, ground state, and first isomer).

they typically are of order $10 \mathrm{mb}$. Our calculations over-predict the $(n, \alpha)$ cross sections by factors of- 

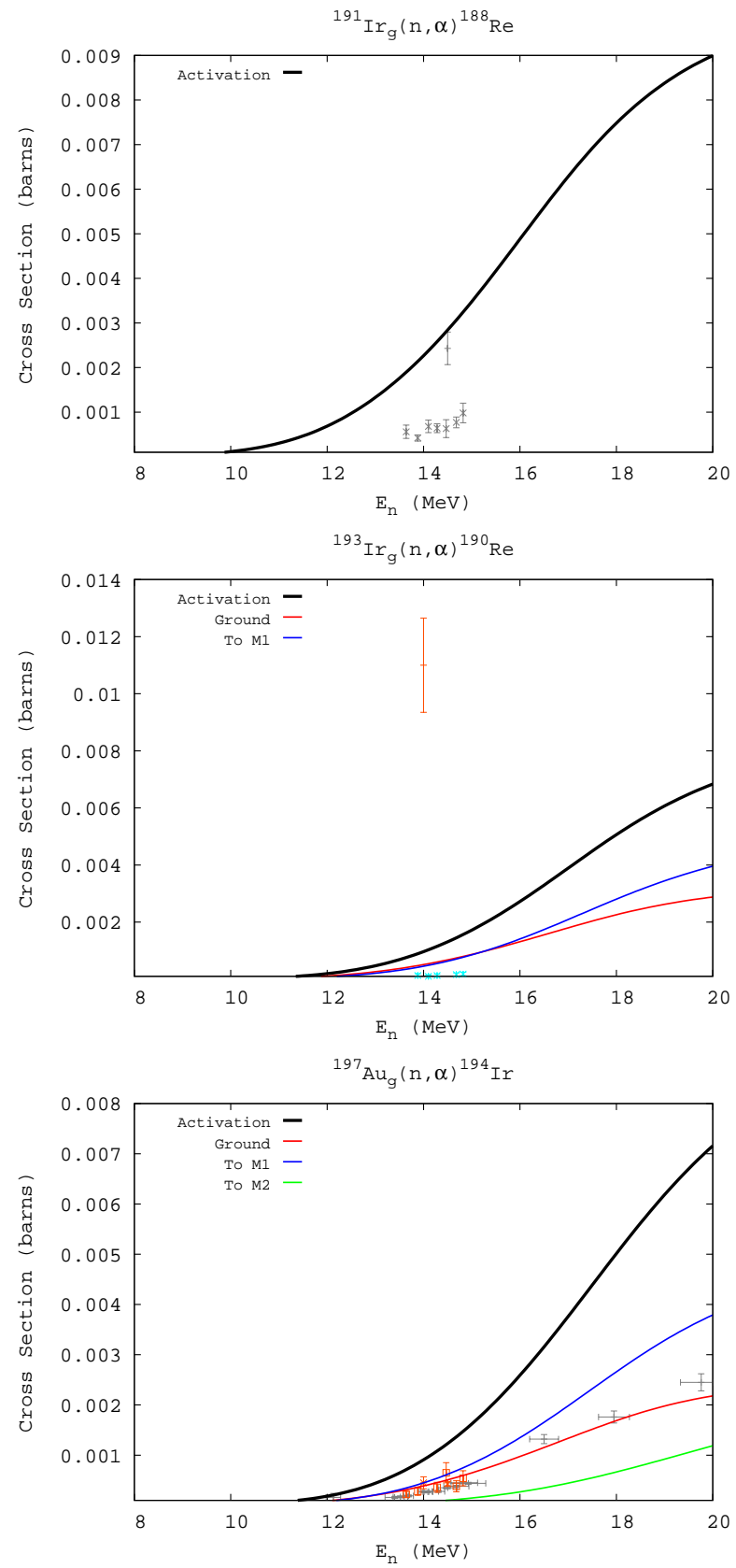

Fig. 17.- Calculated vs. measured $(\mathrm{n}, \alpha)$ cross sections on ${ }^{191,193} \mathrm{Ir}$ and ${ }^{197} \mathrm{Au}$. The data is taken from (EXFOR 2006). The black, red, and blue solid lines represent our modeled cross sections (total, leading to the ground state, and leading to the first isomer, respectively). The grey, orange, and light blue data points are measured cross section data (total, ground state, and first isomer).

ten less than two, a reasonable result in this very heavy mass range.

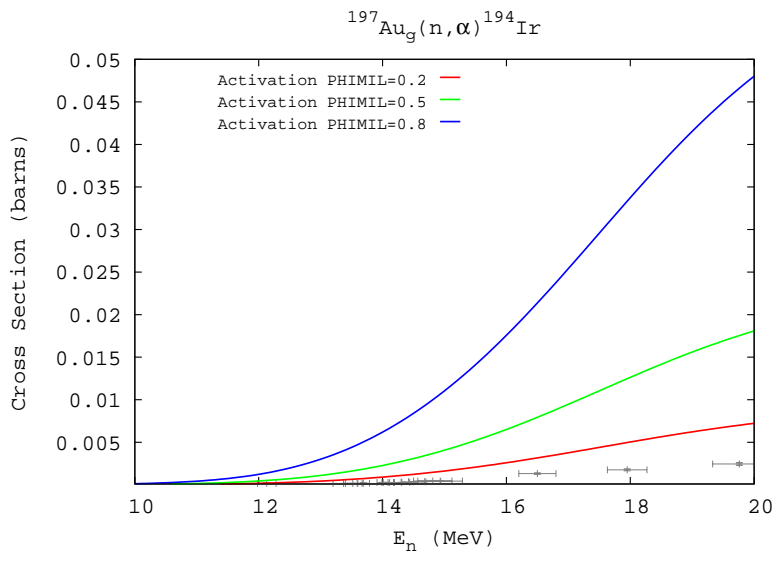

Fig. 18.- Sensitivity of ${ }^{197} \mathrm{Au}(\mathrm{n}, \alpha)$ activation cross section to the alpha pre-formation parameter. The cross section data (grey), represents only activation data. and is taken from (EXFOR 2006). The calculated cross sections shown are for PHIMIL $=0.2($ red $), 0.5($ green $)$, and 0.8 (blue).

\subsection{Sensitivity Studies}

We now illustrate the sensitivity of our modeled results to variations in the input parameters developed in $\S 3$.

\subsubsection{Sensitivity to the Pre-Equilibrium Cross Section}

As stated in $\S 2.4$, the simple exciton model has two free parameters which may be tuned to replicate experimental cross sections. One of these parameters affects alpha emission only and will be presented in the next section. The other parameter, $\langle F M\rangle$, scales the average effective matrix element for residual interactions.

We show the sensitivity of modeled $(\mathrm{n}, 2 \mathrm{n})$ cross sections to averaged experimental activation cross sections at $14.7 \mathrm{MeV}$ to the $\langle F M\rangle$ parameter in Table 4. As in Table 3, the second column shows the number of experimental points entering the average, the third and fourth columns the average value and its variation. The other five columns show the variation of the $(\mathrm{n}, 2 \mathrm{n})$ cross section calculated using values of $\langle F M\rangle=100,150,200,250$, and $300 \mathrm{MeV}$. Based on these results we find that $\langle F M\rangle=200 \mathrm{MeV}$ optimally replicates measured cross sections in this region of interest. 
Table 4: Sensitivity of select $14.7 \mathrm{MeV}(\mathrm{n}, 2 \mathrm{n})$ cross sections to the $\langle F M\rangle$ parameter.

\begin{tabular}{ccccccccc}
\hline \hline${ }^{A} Z$ & $\mathrm{~N}$ & $\bar{\sigma}$ & Dev. & $\sigma_{100}$ & $\sigma_{150}$ & $\sigma_{200}$ & $\sigma_{250}$ & $\sigma_{300}$ \\
\hline${ }^{186} \mathrm{Os}$ & 2 & 1.787 & 0.312 & 1.881 & 2.022 & 2.109 & 2.168 & 2.211 \\
${ }^{192} \mathrm{Os}$ & 5 & 2.135 & 0.142 & 2.055 & 2.143 & 2.194 & 2.228 & 2.252 \\
${ }^{191} \mathrm{Ir}$ & 1 & 1.760 & 0.000 & 1.896 & 2.039 & 2.126 & 2.185 & 2.226 \\
${ }^{193} \mathrm{Ir}$ & 1 & 1.970 & 0.000 & 1.920 & 2.056 & 2.138 & 2.194 & 2.234 \\
${ }^{192} \mathrm{Pt}$ & 3 & 2.019 & 0.014 & 1.901 & 2.043 & 2.128 & 2.183 & 2.223 \\
${ }^{198} \mathrm{Pt}$ & 4 & 1.842 & 0.095 & 2.232 & 2.305 & 2.346 & 2.372 & 2.390 \\
${ }^{197} \mathrm{Au}$ & 31 & 2.140 & 0.135 & 1.920 & 2.066 & 2.154 & 2.214 & 2.256 \\
\hline
\end{tabular}

\subsubsection{Sensitivity to the Alpha Pre-formation Pa- rameter}

The other free parameter in the exciton model which we tune to match measured cross section data describes the tendency of nucleons to preform alpha clusters in the nucleus. This parameter, $\phi$ (PHIMIL), is expected to have a value somewhere between 0.1 to 0.8 . In Figure 18 we present the sensitivity of the ${ }^{197} \mathrm{Au}(\mathrm{n}, \alpha)$ activation cross section to this parameter. The data leading to the ground state and isomers has been left on the plot.

No value of $\phi$ accurately predicts the measured activation cross section. We ascribe this to the choice of $\alpha$-particle optical model potential, which could be off by a factor of 4 or more. Much better agreement with the ground state data (which overlaps the activation data, see Figure 14), justifies a choice of $\phi=0.2$ (the lowest value). Because the alpha channel is small compared to other exit channels, the effect on other cross sections, such as $(\mathrm{n}, 2 \mathrm{n})$ and $(\mathrm{n}, \mathrm{p})$ is negligible, and the only other calculated reaction channel that will show any sensitivity to this parameter is the $(\mathrm{n}, \mathrm{n} \alpha)$ channel. Since charged particle reactions are so small in this heavy mass system and are not part of the cross section set we do not see this as a major problem for UGT analysis.

\subsection{Calculated Channels by Target}

In Appendix $\mathrm{C}$ we show all of the calculated neutron induced channels leading out of the ground state for the targets of iridium and gold included in this set. In general, variations in a given cross section from target to target are small, although odd-even effects are apparent, especially in the capture reactions. All are arranged with even neutron numbers on the right panel and odd neutron numbers on the left, increasing with A from bottom to top.

These plots aid the evaluation of which reaction channels are the most important. For neu- trons with low incident energies (below the $(\mathrm{n}, 2 \mathrm{n})$ threshold), the (n, $\gamma$ ) capture reaction is dominant. Thresholds for the $(\mathrm{n}, \mathrm{p})$ cross sections in this mass range always occur at an energy in excess of that where the $(\mathrm{n}, \gamma)$ reaction drops below $1 \mathrm{mb}$. At energies around $14 \mathrm{MeV}$ the (n,2n) cross sections always dominate over $(\mathrm{n}, \mathrm{p})$ by roughly two orders of magnitude, as does (n,3n) at higher energies.

Of course the particle fluences play a dominant role in determining the most important cross sections for RADCHEM. More specific details will be disclosed in a classified analysis presented in a subsequent paper.

\subsection{Comparison to LANL RADCHEM cross sections}

In Appendix B.6 we compare select modeled iridium cross sections to their LANL counterparts (Chadwick et. al. 2007). These particular cross sections were chosen by virtue of their having experimental data to compare them against as well as being the first order production reactions for the relevant radiochemical products measured in the UGT program 1.2.

The important $(\mathrm{n}, 2 \mathrm{n})$ reactions on both loaded iridium isotopes are in very good agreement, with only the value at threshold being discrepant. Both compare quite well with the data around $14 \mathrm{MeV}$ and modestly well at higher energies, although the LLNL ${ }^{193} \operatorname{Ir}(\mathrm{n}, 2 \mathrm{n})^{192} \operatorname{Ir}$ cross section appears to be closer to the data above this. The cross section values at $14.1 \mathrm{MeV}$ for ${ }^{191} \operatorname{Ir}(\mathrm{n}, 2 \mathrm{n})$ and ${ }^{193} \operatorname{Ir}(\mathrm{n}, 2 \mathrm{n})$ are 2.14 and 2.18 barns (LANL), and 2.11 and 2.14 (LLNL), respectively.

The LANL ${ }^{191} \operatorname{Ir}(\mathrm{n}, 3 \mathrm{n}){ }^{189} \operatorname{Ir}$ cross section appears consistently higher than experiment suggests from threshold to $20 \mathrm{MeV}$, although the difference to LLNL decreases at higher energies. It appears the reaction thresholds are different, ours are calculated using the measured mass excess data in (Tuli 2000)(Table 5 Appendix A). 
A cross section is provided for ${ }^{193} \operatorname{Ir}\left(\mathrm{n}, \mathrm{n}^{\prime}\right){ }^{193_{m}{ }^{1}} \mathrm{Ir}$ (although one to depopulate the isomer is not included). Compared to data near $8 \mathrm{MeV}$ both LLNL and LANL cross sections model the data well, below this they differ at $.5 \mathrm{MeV}$ by almost a factor of two. The recent LANL evaluation provides a comparison to a more recent measurement which used the LANL GNASH statistical model in its evaluation.

For the capture reactions on loaded ${ }^{191,193}$ Ir the modeled cross sections from LANL clearly better replicate the experimental data shown above 100 $\mathrm{keV}$, due in large part to the DEGAS model for pre-equilibrium included there. For the crucial range between 1 and $100 \mathrm{keV}$ both LANL and LLNL modeled cross sections are in very good agreement with the data. When one considers the Maxwellian averaged cross sections ( $\S$ 4.1.2) for ${ }^{191} \operatorname{Ir}(\mathrm{n}, \gamma)$ and ${ }^{193} \operatorname{Ir}(\mathrm{n}, \gamma)$ at $30 \mathrm{keV}$, the LANL MACS are 1336 and $864 \mathrm{mb}$ which matches the experimental data $(1350 \pm 43$ and $994 \pm 70 \mathrm{mb})$ to within $1 \%$ and $13 \%$, respectively. The LLNL MACS (1297 and $906 \mathrm{mb}$ ) match the experimental data to within $4 \%$ and $9 \%$. This is good agreement for both efforts and well within the envelope of success that we have noted in Table 2.

Overall this is good news as the LANL set has recently been extensively evaluated against not only traditional accelerator based cross section measurements but also critical assembly and Bethe sphere measurements carried out at LANL, for which the agreement is compelling.

\section{Conclusions}

We have developed new neutron induced cross section detector sets for radiochemical diagnostics for $\operatorname{Ir}(Z=77,187 \leq A \leq 195)$ and $\mathrm{Au}(Z=79$, $193 \leq A \leq 199)$. The theory and implementation of the Hauser-Feshbach model were described ( $(2)$, along with the details of the local systematics used to create a set of input parameters that reflect the latest available experimental data in the local region of interest ( $\S 3)$. Modeled cross sections were compared to available experimental cross sections for the loaded detector elements, as well as other stable targets in the region. Sensitivity to reasonable variations in the input models and parameters was explored (\$4.2).

Overall we consider the modeling effort to be quite successful, as our calculated cross sections agree favorably with experimentally measured ones in this region of interest. In particular, we have demonstrated an ability to calculate $(n, 2 n)$ cross sections to about $12 \%$ accuracy (Section 4.1.4), and (n, $\gamma)$ cross sections to within roughly 15-20\% accuracy (Sections 4.1.1 and 4.1.2). Cross sections for reactions involving charged particles were shown to be very small compared to the dominant $(\mathrm{n}, 2 \mathrm{n}),(\mathrm{n}, 3 \mathrm{n}),\left(\mathrm{n}, \mathrm{n}^{\prime}\right)$, and $(\mathrm{n}, \mathrm{g})$ reactions that make up this set although our calculations did compare favorably to them ( $\S$ 4.1.6). A select set of our modeled cross sections on the stable isotopes of Ir compare quite well against a recent effort by LANL (Chadwick et. al. 2007) which was evaluated against traditional accelerator based cross section data as well as critical assembly and Bethe sphere measurements.

In our attempts to model cross sections it should be kept in mind that we are considering compound nuclear systems for which the important input parameters to our reaction model (e.g. those that affect level densities and photontransmission coefficients) are often determined by normalization to experimental data (e.g. from resonance analysis), and so one would expect comparisons to measured capture cross sections to be good. Since these compound nuclei often bracket the systems of most interest to us, namely those which account for the dominant destruction reactions, our systematics should reasonably provide for similar agreement.

This work was performed under the auspices of the U.S. Department of Energy by the University of California Lawrence Livermore National Laboratory in part under contract W-7405-ENG-48 and in part under Contract DE-AC52-07NA27344. 


\section{REFERENCES}

Audi, G., Wapstra, A.H., and Thibault, C., "The AME2003 atomic mass evaluation (II). Tables, graphs, and references", Nuc. Phys. A 729, 337 (2003).

Avrigeanu, V., Hodgson, P.E., and Avrigeanu, M., "Global optical potentials for emitted alpha particles", Phys. Rev. C 49, 2136 (1994).

Avrigeanu, M. and Avrigeanu, V., "Recent improvements of the STAPRE-H95 pre-equilibrium and statistical model code", Report NP-86-1995, IPNE-Bucharest (1995). http://www.nea.fr/abs/html/iaea0971.html

Bao, Z. Y., Beer, H., Kappeler, F., Voss, F., \& Wisshak, K., "Neutron cross sections for nucleosynthesis studies", Atomic Data \& Nuclear Data Tables, 76, 70 (2000).

Bauer, R., \& Kelley, K. private communication (2007).

Beer, H., Voss, F., \& Winters, R. R., "On the calculation of Maxwellian-averaged capture cross sections", ApJS, 80, 403 (1992).

Belgya, T., Bersillon, O., Copote Noy, R., Fukahori, T., Zhigang, G., Goriely, S., Herman, M., Ignatyuk, A.V., Kailas, S., Koning, A.J., Oblozinsky, P., Plujko, V, and Young, P.G., "Handbook for calculations of nuclear reaction data, RIPL-2", IAEA, Vienna (2005).

Bohr, A., \& Mottelson, B. Nuclear Structure Vol. 1, Single-Particle Motion, World Scientific, 1998

Chadwick, M., "The GNASH statistical model code", 1998. http://www.nea.fr/abs/html/psr-0125.html

Cline, C. K., "The Pauli exclusion principle in pre-equilibrium decay", Nucl. Phys. A195, 353 (1972).

Cline, C.K., and Blann, M., "The pre-equilibrium statistical model: description of the nuclear equilibration process and parameterization of the model", Nuc. Phys. A 172, 225 (1971).

Dietrich, F.S., 2001, private communication.

Experimental Nuclear Reaction Data File, Brookhaven National Laboratory, US Dept. of Energy (2006). http://www.nndc.bnl.gov/exfor3/

Evaluated Nuclear Structure Data File, Brookhaven National Laboratory NNDC, US Dept. of Energy (2006). http://www.nndc.bnl.gov/ensdf/

Gardner, D.G., "Model calculations as one means of satisfying the neutron cross section requirements of the CTR program", Invited paper presented at the Conference on Nuclear Cross Sections and Technology, Washington, D.C., March 3-7, 1975.

Garrett, P. et. al. 1994 Nucl. Phys. A568, 445.

Garrett, P. et. al. 1994 Nucl. Phys. A571, 103.

Garrett, P. et. al. 1995 Nucl. Phys. A581, 267.

Garrett, P. et. al. 1996 Nucl. Phys. A611, 68.

Garrett, P. et. al. 2000 Nucl. Phys. A662, 235.

Gilbert, A. and Cameron, A.G.W., "A composite nuclear-level density formula with shell corrections", Can. J. Phys., 43, 1446 (1965).

Hauser, W. and Feshbach, H., "The inelastic scattering of neutrons", Phys. Rev. 87, 366 (1952).

Hilaire, S., Lagrange, Ch., and Koning, A. J., "Comparisons between various width fluctuation correction factors for compound nucleus reactions" Ann. of Phys. 306, 209 (2003). 
Hoffman, R. D., Rauscher, T., Woosley, S. E. \& Thielemann, F.-K., "The reaction rate sensitivity of nucleosynthesis in Type II supernovae", ApJ, 521, 735 (1999).

Hoffman, R.D., Dietrich, F.S., Bauer, R., Kelley, K., and Mustafa, M.G., "Neutron and charged-particle induced cross sections for radiochemistry in the region of bromine and krypton", UCRL-TR-205563, LLNL (2004a).

Hoffman, R.D., Dietrich, F.S., Bauer, R., Kelley, K., and Mustafa, M.G., "Neutron and charged-particle induced cross sections for radiochemistry in the region of iodine and xenon", UCRL-TR-206721, LLNL (2004b).

Hoffman, R.D., Kelley, K., Dietrich, F.S., Bauer, R., and Mustafa, M.G., "Neutron and charged-particle induced cross sections for radiochemistry in the region of samarium, europium and gadolinium", UCRLTR-211558, LLNL (2004c).

Hoffman, R.D., Kelley, K., Dietrich, F.S., Bauer, R., and Mustafa, M.G., "Modeled neutron and chargedparticle induced nuclear reaction cross sections for radiochemistry in the region of yttrium, zirconium, niobium, and molybdenum", UCRL-TR-222275, LLNL (2006c).

Hofmann, H.M., Richert, J., Tepel, J. W., and Weidenmuller, H.A., "Direct reactions and Hauser-Feshbach theory" Ann. of Phys. 90, 403 (1975).

Iljinov, A.S., Mebel, M.V., Bianchi, N., De Sanctis, E., Guaraldo, C., Lucherini, V., Muccifora, V., Polli, E., Reolon, A.R., and Rossi, P., "Phenomenological statistical analysis of level densities, decay widths and lifetimes of excited nuclei", Nucl. Phys. A 543, 517 (1992).

Keisch, B., "Yield ratios of isomers produced by neutron activation", Phys. Rev. 129, 769 (1963).

Kelley, K., Hoffman, R.D., Dietrich, F.S., Bauer, R., and Mustafa, M.G., "'Neutron and charged-particle induced cross sections for radiochemistry for isotopes of scandium, titanium, vanadium, chromium, manganese, and iron", UCRL-TR-211668, LLNL (2005).

Kelley, K., Hoffman, R.D., Dietrich, F.S., and Mustafa, M.G., "Neutron induced cross sections for radiochemistry for isotopes of arsenic", UCRL-TR-218181, LLNL (2006a).

Kelley, K., Hoffman, R.D., Dietrich, F.S., and Mustafa, M.G., "Neutron induced cross sections for radiochemistry for isotopes of nickel, copper, and zinc", UCRL-TR-221759, LLNL (2006b).

Kopecky, J. and Uhl, M., "Test of gamma-ray strength functions in nuclear reaction model calculations", Phys. Rev. C 41, 1941 (1990).

Lohr, J.M. and Haeberli, W., "Elastic scattering of 9-13 MeV vector polarized deuterons", Nuc. Phys. A 232, 381 (1974).

Mahaux, C. and Weidenmüller, H.A., "Recent developments in compound-nucleus theory", Ann. Rev. Part. Nucl. Sci. 29, 1 (1979).

Milazzo-Colli, L., and Braga-Marcazzan, G.M., " $\alpha$-emission by pre-equilibrium processes in (n, $\alpha$ ) reactions", Nuc. Phys. A 210, 297 (1973).

Moldauer, P. A., "Evaluation of the fluctuation enhancement factor" Phys. Rev. C 14, 764 (1976).

Möller, P., Nix, J.R., Myers, W.D., and Swiatecki, W.J., "Nuclear ground-state masses and deformations" At. Data \& Nuc. Data Tables 59, 185 (1995).

Mughabghab, S.F., Divadeenam, M., and Holden, N.E., Neutron Cross Sections, Vols. 1 and 2, Academic Press (1981).

Nethaway, D.R., "The cross-section sets used with the Watusi program", A-Division memo, LLNL (5 Nov. 1998). 
Rauscher, T., Thielemann, F.-K., and Kratz, K.-L., "Nuclear level density and the determination of thermonuclear rates for astrophysics", Phys. Rev. C 56, 1613 (1997).

Raynal, J. "ECIS96", Proceedings of the Specialists' Meeting on the Nucleon Nucleus Optical Model up to $200 \mathrm{MeV}$, 13-15 November 1996, Bruyeres-le-Chatel, France. http://www.nea.fr/html/science/om200/raynal.pdf

Chadwick, M.B., Frankle, S., Trellue, H., Talou, P., Kawano, T., Young, P.G., MacFarlane, R.F., and Wilkerson, C. W., Nuclear Data Sheets, 108, Number 12, 2716 (2007).

Tuli, J.K., "Nuclear wallet cards", 6th Ed., Brookhaven National Laboratory, U.S. Dept. of Energy (2000).

Uhl, M., and Strohmaier, B., "STAPRE: A computer code for particle induced activation cross sections and related quantities", IRK-Vienna Report IRK-76/01 (1976, Upd. 1978).

Vonach, H., "User's manual for the code STAPRE as implemented at Lawrence Livermore National Laboratory", UCID-19549, LLNL (1982).

Wapstra, A.H., Audi, G., and Thibault, C., "The AME2003 atomic mass evaluation (I). Evaluation of input data, adjustment procedures", Nuc. Phys. A 729, 129 (2003).

Williams, F.C. Jr., "Intermediate state transition rates in the Griffin model", Phys. Lett. 31B, 184 (1970).

This 2-column preprint was prepared with the AAS LATEX macros v5.0. 


\section{A. Cross Sections Included in the Detector Sets}

Cross sections for $(\mathrm{n}, 3 \mathrm{n}),(\mathrm{n}, 2 \mathrm{n}),(\mathrm{n}, \gamma)$, and $\left(\mathrm{n}, \mathrm{n}^{\prime}\right)$ reactions on the targets listed below constitue the existing RADCHEM data sets Ir0188 and Au1085. The new evaluation also includes (n,p) cross sections. Isomer spin assignments reflect modern values with parenthesis indicating an uncertain assigment. The majority of the reactions in Ir0188 and Au1085 were calculated between 1985 and 1988. A few had been scaled to match measured cross sections at or around $14.1 \mathrm{MeV}$ of incident energy.

\section{A.1. Iridium and Gold Detector Sets}

Table 5:: Neutron induced reactions calculated for new iridium and gold sets

\begin{tabular}{|c|c|c|c|c|c|c|c|}
\hline${ }^{A} Z$ & lifetime & $\overline{\mathrm{J}^{\pi}}$ & $\overline{(\overline{(n, 3 n)}}$ & $\overline{(\mathrm{n}, 2 \mathrm{n})}$ & $\overline{\left(n, n^{\prime}\right)}$ & $\overline{(\mathrm{n}, \gamma)}$ & $\overline{(n, p)}$ \\
\hline${ }^{187} \mathrm{Ir}$ & $10.5 \mathrm{~h}$ & $1.5+$ & o & $\circ$ & o & o & o \\
\hline${ }^{187} \operatorname{Ir}_{m 1}$ & $30.3 \mathrm{~ms}$ & $4.5-$ & O & ○ & o & o & 0 \\
\hline${ }^{188} \mathrm{Ir}$ & $41.5 \mathrm{~h}$ & $1-$ & ○ & ○ & ○ & ○ & 0 \\
\hline${ }^{188} \operatorname{Ir}_{m 1}$ & $4.20 \mathrm{~ms}$ & $11-$ & $\circ$ & o & ○ & ○ & ○ \\
\hline${ }^{189} \mathrm{Ir}$ & $13.2 \mathrm{~d}$ & $1.5+$ & ○ & ○ & o & ○ & ○ \\
\hline${ }^{189} \operatorname{Ir}_{m 1}$ & $13.3 \mathrm{~ms}$ & $5.5-$ & ○ & ○ & ○ & ○ & ○ \\
\hline${ }^{190} \mathrm{Ir}$ & $11.78 \mathrm{~d}$ & 4- & $\circ$ & $\circ$ & ○ & ○ & ○ \\
\hline${ }^{190} \mathrm{Ir}_{m 1}$ & $1.12 \mathrm{~h}$ & $(1-)$ & $\circ$ & $\circ$ & $\circ$ & ○ & ○ \\
\hline${ }^{190} \operatorname{Ir}_{m 2}$ & $3.09 \mathrm{~h}$ & (11-) & $\circ$ & ○ & $\circ$ & ○ & ○ \\
\hline${ }^{191} \mathrm{Ir}$ & $37.3 \%$ & $1.5+$ & $\circ$ & ○ & ○ & $\circ$ & ○ \\
\hline${ }^{191} \operatorname{Ir}_{m 1}$ & $4.94 \mathrm{~s}$ & $5.5-$ & ○ & ○ & o & ○ & ○ \\
\hline${ }^{192} \mathrm{Ir}$ & $73.83 \mathrm{~d}$ & $4+$ & $\circ$ & $\circ$ & 0 & O & ○ \\
\hline${ }^{192} \operatorname{Ir}_{m 1}$ & $1.45 \mathrm{~m}$ & $1-$ & ० & ○ & ○ & o & ○ \\
\hline${ }^{192} \operatorname{Ir}_{m 2}$ & $241 \mathrm{y}$ & $(11-)$ & ○ & ○ & ○ & ० & ○ \\
\hline${ }^{193} \mathrm{Ir}$ & $62.7 \%$ & $1.5+$ & $\circ$ & ○ & ○ & ○ & ○ \\
\hline${ }^{193} \operatorname{Ir}_{m 1}$ & $10.53 \mathrm{~d}$ & $5.5-$ & ○ & ○ & ○ & ○ & ○ \\
\hline${ }^{194} \mathrm{Ir}$ & $19.28 \mathrm{~h}$ & $1-$ & ○ & ○ & ○ & ○ & ○ \\
\hline${ }^{194} \operatorname{Ir}_{m 1}$ & $31.8 \mathrm{~ms}$ & $4+$ & ○ & ○ & o & o & o \\
\hline${ }^{194} \operatorname{Ir}_{m 2}$ & $0.47 \mathrm{y}$ & $(11-)$ & $\circ$ & $\circ$ & $\circ$ & ○ & $\circ$ \\
\hline${ }^{195} \mathrm{Ir}$ & $2.50 \mathrm{~h}$ & $1.5+$ & $\circ$ & ○ & ○ & ○ & ○ \\
\hline${ }^{195} \operatorname{Ir}_{m 1}$ & $3.80 \mathrm{~h}$ & $5.5-$ & ○ & ○ & ○ & ○ & ○ \\
\hline${ }^{193} \mathrm{Au}$ & $17.65 \mathrm{~h}$ & $1.5+$ & $\circ$ & o & ○ & $\circ$ & ○ \\
\hline${ }^{193} \mathrm{Au}_{m 1}$ & $3.90 \mathrm{~s}$ & $5.5-$ & $\circ$ & ○ & ○ & ○ & ○ \\
\hline${ }^{194} \mathrm{Au}$ & $38.02 \mathrm{~h}$ & $1-$ & $\circ$ & ○ & ○ & ○ & ○ \\
\hline${ }^{194} \mathrm{~A} u_{m 1}$ & $0.60 \mathrm{~s}$ & $(5+)$ & ○ & o & o & ○ & o \\
\hline${ }^{194} \mathrm{Au}_{m 2}$ & $0.42 \mathrm{~s}$ & $(11-)$ & $\circ$ & $\circ$ & $\circ$ & ○ & ○ \\
\hline${ }^{195} \mathrm{Au}$ & $186.10 \mathrm{~d}$ & $1.5+$ & ○ & ○ & ○ & ○ & ○ \\
\hline${ }^{195} \mathrm{Au}_{m 1}$ & $30.50 \mathrm{~s}$ & $5.5-$ & $\circ$ & ○ & ○ & ○ & ○ \\
\hline${ }^{196} \mathrm{Au}$ & $6.17 \mathrm{~d}$ & $2-$ & $\circ$ & $\circ$ & ○ & ○ & ० \\
\hline${ }^{196} \mathrm{Au} u_{m 1}$ & $8.10 \mathrm{~s}$ & $5+$ & ○ & ○ & ○ & ○ & ० \\
\hline${ }^{196} \mathrm{Au} u_{m 2}$ & $9.60 \mathrm{~h}$ & $12-$ & ० & ○ & ○ & ○ & o \\
\hline${ }^{197} \mathrm{Au}$ & $100 \%$ & $1.5+$ & ० & $\circ$ & ○ & $\circ$ & ○ \\
\hline${ }^{197} \mathrm{Au}_{m 1}$ & $7.73 \mathrm{~s}$ & $5.5-$ & ○ & $\circ$ & ○ & ○ & ○ \\
\hline${ }^{198} \mathrm{Au}$ & $2.70 \mathrm{~d}$ & $2-$ & $\circ$ & $\circ$ & $\circ$ & $\circ$ & ○ \\
\hline${ }^{198} \mathrm{~A} u_{m 1}$ & $2.27 \mathrm{~d}$ & $(12-)$ & ○ & $\circ$ & $\circ$ & ○ & ○ \\
\hline${ }^{199} \mathrm{Au}$ & $3.14 \mathrm{~d}$ & $1.5+$ & ○ & ○ & ○ & o & o \\
\hline${ }^{199} \mathrm{Au}_{m 1}$ & $0.44 \mu \mathrm{s}$ & $5.5-$ & $\circ$ & $\circ$ & $\circ$ & $\circ$ & 0 \\
\hline
\end{tabular}




\section{A.2. Q-Values for Reactions Studied}

Here we present the $\mathrm{Q}$-values (in $\mathrm{MeV}$ ) for each of the reactions included in this study. The values provided in this table are for reactions proceeding from the ground state of the target to the ground state of the residual. The Q-value for reactions proceeding from/to other states can be obtained by adding the energy of the target state to the given Q-value and subtracting the energy of the residual state. The values are calculated from our adopted mass excesses, as described in section 3.1.1. Inelastic scattering reactions such as (n,n') have thresholds equal to the energy of the first excited state. See the discrete level schemes (Appendix A.3).

Table 6:: Reaction Q-values

\begin{tabular}{|c|c|c|c|c|c|}
\hline Target & $Q_{(n, \gamma)}$ & $Q_{(n, 2 n)}$ & $Q_{(n, 3 n)}$ & $Q_{(n, p)}$ & $Q_{(n, \alpha)}$ \\
\hline${ }^{186} \mathrm{Os}$ & 6.293 & -8.261 & -14.887 & -0.286 & 9.014 \\
\hline${ }^{187} \mathrm{Os}$ & 7.989 & -6.293 & -14.555 & 0.779 & 10.131 \\
\hline${ }^{188} \mathrm{Os}$ & 5.920 & -7.989 & -14.283 & -1.339 & 7.895 \\
\hline${ }^{189} \mathrm{Os}$ & 7.791 & -5.920 & -13.910 & -0.227 & 9.169 \\
\hline${ }^{190} \mathrm{Os}$ & 5.758 & -7.791 & -13.712 & -2.358 & 6.845 \\
\hline${ }^{191} \mathrm{Os}$ & 7.558 & -5.758 & -13.550 & -1.263 & 7.920 \\
\hline${ }^{192} \mathrm{Os}$ & 5.585 & -7.558 & -13.317 & -3.210 & 5.243 \\
\hline${ }^{187} \mathrm{Ir}$ & 6.682 & -8.621 & -15.526 & 2.285 & 10.151 \\
\hline${ }^{188} \mathrm{Ir}$ & 8.197 & -6.682 & -15.304 & 3.592 & 11.139 \\
\hline${ }^{189} \mathrm{Ir}$ & 6.324 & -8.197 & -14.880 & 1.315 & 9.121 \\
\hline${ }^{190} \mathrm{Ir}$ & 8.072 & -6.324 & -14.522 & 2.782 & 10.156 \\
\hline${ }^{191} \mathrm{Ir}$ & 6.198 & -8.072 & -14.397 & 0.468 & 7.955 \\
\hline${ }^{192} \mathrm{Ir}$ & 7.771 & -6.198 & -14.271 & 1.828 & 8.789 \\
\hline${ }^{193} \mathrm{Ir}$ & 6.067 & -7.771 & -13.970 & -0.358 & 6.678 \\
\hline${ }^{194} \mathrm{Ir}$ & 7.231 & -6.067 & -13.839 & 0.685 & 7.464 \\
\hline${ }^{195} \mathrm{Ir}$ & 5.833 & -7.231 & -13.299 & -1.218 & 5.844 \\
\hline${ }^{190} \mathrm{Pt}$ & 6.437 & -8.911 & -15.645 & 0.166 & 9.542 \\
\hline${ }^{191} \mathrm{Pt}$ & 8.677 & -6.437 & -15.349 & 1.800 & 11.094 \\
\hline${ }^{192} \mathrm{Pt}$ & 6.255 & -8.677 & -15.114 & -0.678 & 8.338 \\
\hline${ }^{193} \mathrm{Pt}$ & 8.370 & -6.255 & -14.932 & 0.838 & 9.874 \\
\hline${ }^{194} \mathrm{Pt}$ & 6.104 & -8.370 & -14.625 & -1.465 & 7.262 \\
\hline${ }^{195} \mathrm{Pt}$ & 7.922 & -6.104 & -14.475 & -0.338 & 8.717 \\
\hline${ }^{196} \mathrm{Pt}$ & 5.846 & -7.922 & -14.027 & -2.427 & 6.380 \\
\hline${ }^{197} \mathrm{Pt}$ & 7.556 & -5.846 & -13.769 & -1.373 & 7.643 \\
\hline${ }^{198} \mathrm{Pt}$ & 5.556 & -7.556 & -13.403 & -3.008 & 5.415 \\
\hline${ }^{193} \mathrm{Au}$ & 6.947 & -8.703 & -15.693 & 1.851 & 8.944 \\
\hline${ }^{194} \mathrm{Au}$ & 8.370 & -6.947 & -15.651 & 3.274 & 10.068 \\
\hline${ }^{195} \mathrm{Au}$ & 6.642 & -8.370 & -15.318 & 1.008 & 7.896 \\
\hline${ }^{196} \mathrm{Au}$ & 8.071 & -6.642 & -15.013 & 2.288 & 9.025 \\
\hline${ }^{197} \mathrm{Au}$ & 6.512 & -8.071 & -14.714 & 0.063 & 7.021 \\
\hline${ }^{198} \mathrm{Au}$ & 7.584 & -6.512 & -14.583 & 1.107 & 7.740 \\
\hline${ }^{199} \mathrm{Au}$ & 6.236 & -7.584 & -14.097 & -0.921 & 5.989 \\
\hline${ }^{196} \mathrm{Hg}$ & 6.785 & -8.838 & -15.739 & 0.096 & 8.283 \\
\hline${ }^{197} \mathrm{Hg}$ & 8.484 & -6.785 & -15.624 & 1.382 & 9.868 \\
\hline${ }^{198} \mathrm{Hg}$ & 6.664 & -8.484 & -15.270 & -0.590 & 7.488 \\
\hline${ }^{199} \mathrm{Hg}$ & 8.028 & -6.664 & -15.149 & 0.330 & 8.746 \\
\hline${ }^{200} \mathrm{Hg}$ & 6.230 & -8.028 & -14.693 & -1.462 & 6.564 \\
\hline${ }^{201} \mathrm{Hg}$ & 7.754 & -6.230 & -14.259 & -0.481 & 7.890 \\
\hline${ }^{202} \mathrm{Hg}$ & 5.992 & -7.754 & -13.985 & -2.164 & 5.692 \\
\hline${ }^{203} \mathrm{Hg}$ & 7.495 & -5.992 & -13.747 & -1.342 & 6.981 \\
\hline${ }^{204} \mathrm{Hg}$ & 5.668 & -7.495 & -13.488 & -3.046 & 4.695 \\
\hline
\end{tabular}




\section{A.3. Modified Discrete Level Schemes}

As stated in section 3.1.2, our discrete level schemes were fully reevaluated. Unless specifically cited in the figure captions the basis for the energy levels and branching ratios was (ENSDF 2006), while the spins and parities were principally from (Belgya et al. 2005). In the following plots, the level energy is listed on the right hand side, and the spin and parity of the level on the left. The $\gamma$-ray transitions are indicated by arrows. Each arrow is labeled with the transition energy, followed by the branching ratio (as a percent) in parenthesis. Isomeric states are indicated by thick black lines. 

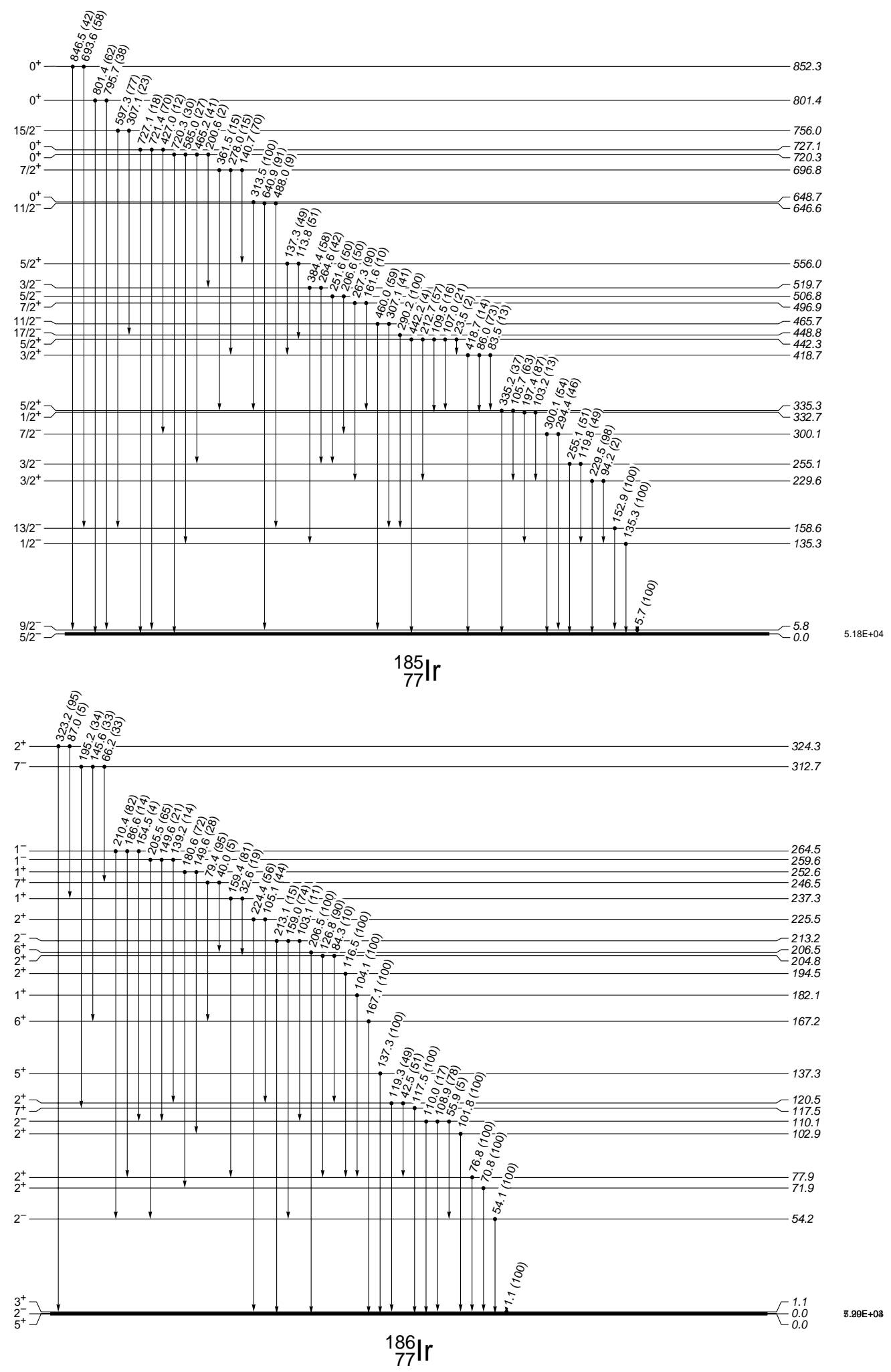

Fig. 19.- Adopted level schemes for ${ }^{185} \mathrm{Ir}$ and ${ }^{186} \mathrm{Ir}$. 

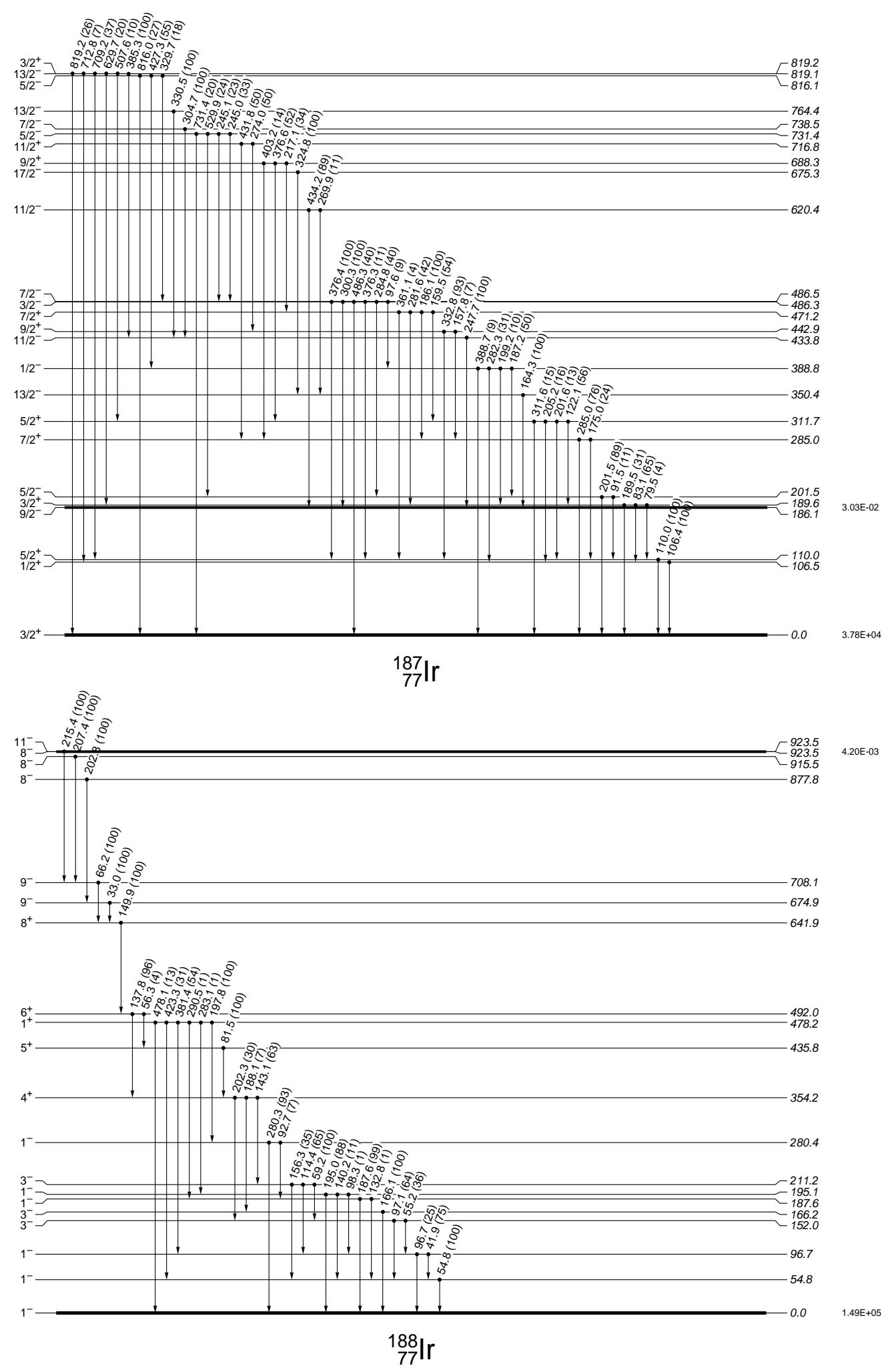

Fig. 20.- Adopted level schemes for ${ }^{187} \mathrm{Ir}$ and ${ }^{188} \mathrm{Ir}$. 

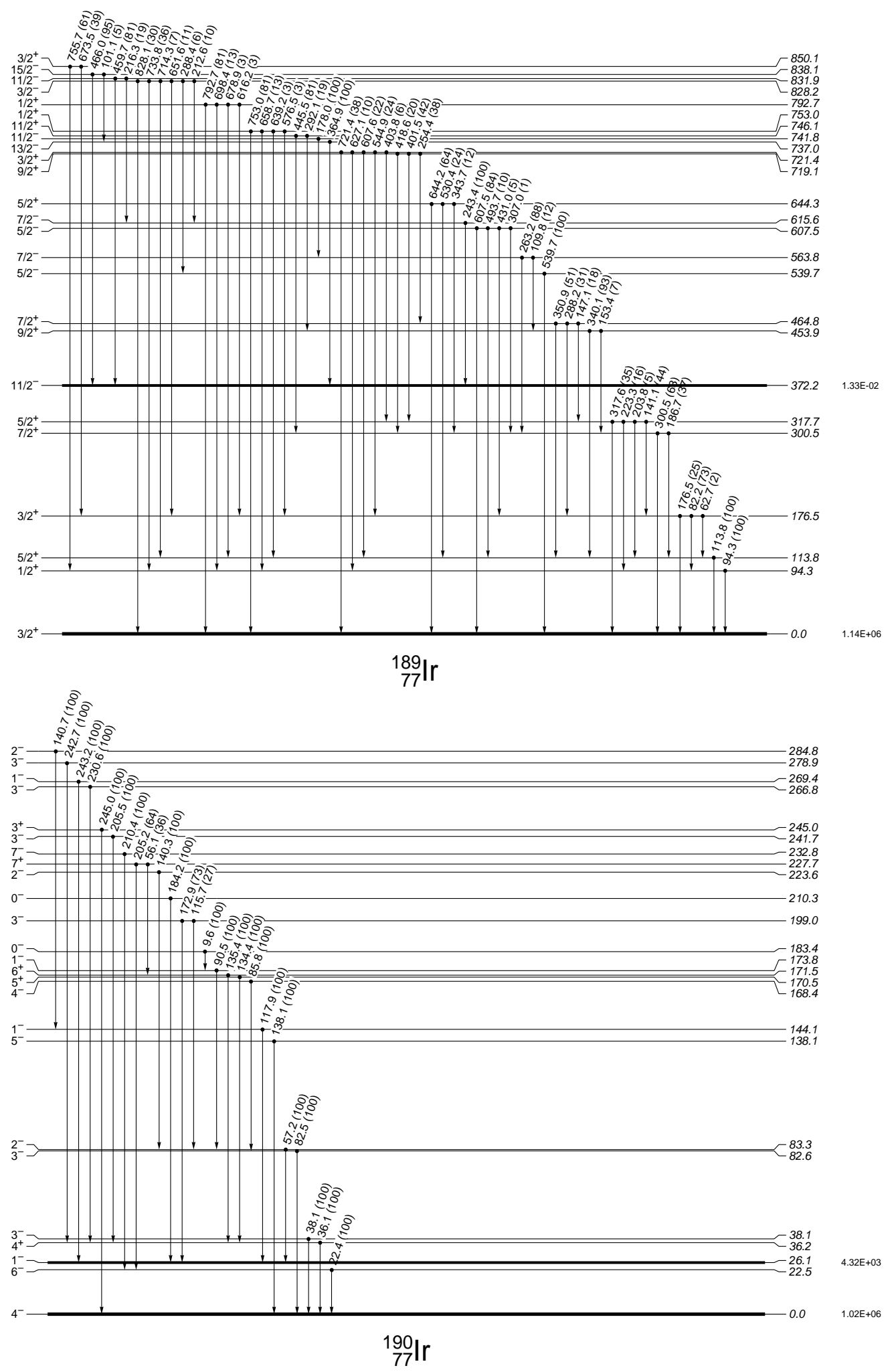

Fig. 21.- Adopted level schemes for ${ }^{189} \operatorname{Ir}$ and ${ }^{190} \operatorname{Ir}(3 ; 4 ; 5)$. 

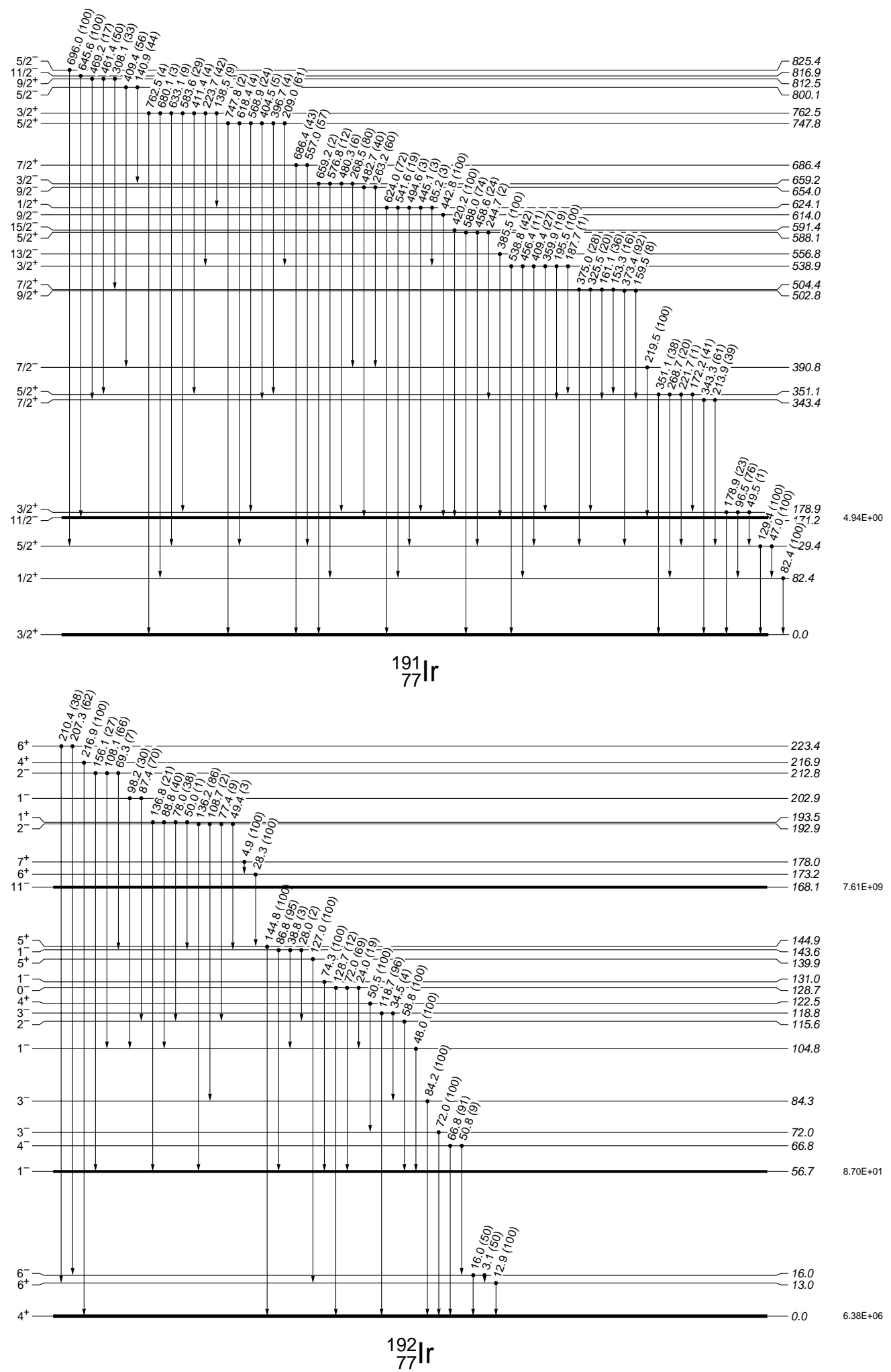

Fig. 22.- Adopted level schemes for ${ }^{191} \operatorname{Ir}$ and ${ }^{192} \operatorname{Ir}(1)$. 

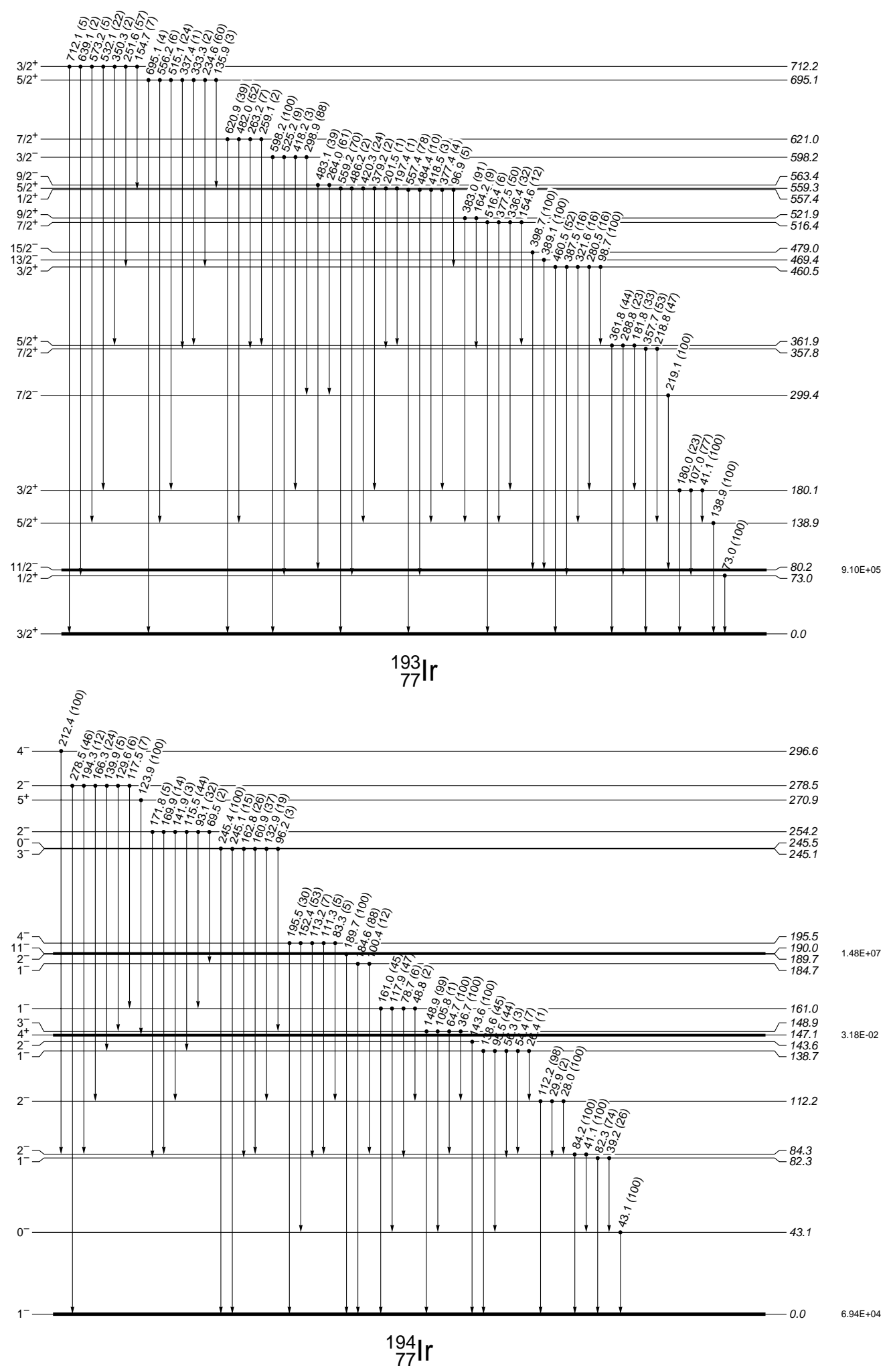

Fig. 23. - Adopted level schemes for ${ }^{193} \operatorname{Ir}$ and ${ }^{194} \operatorname{Ir}(2)$. 


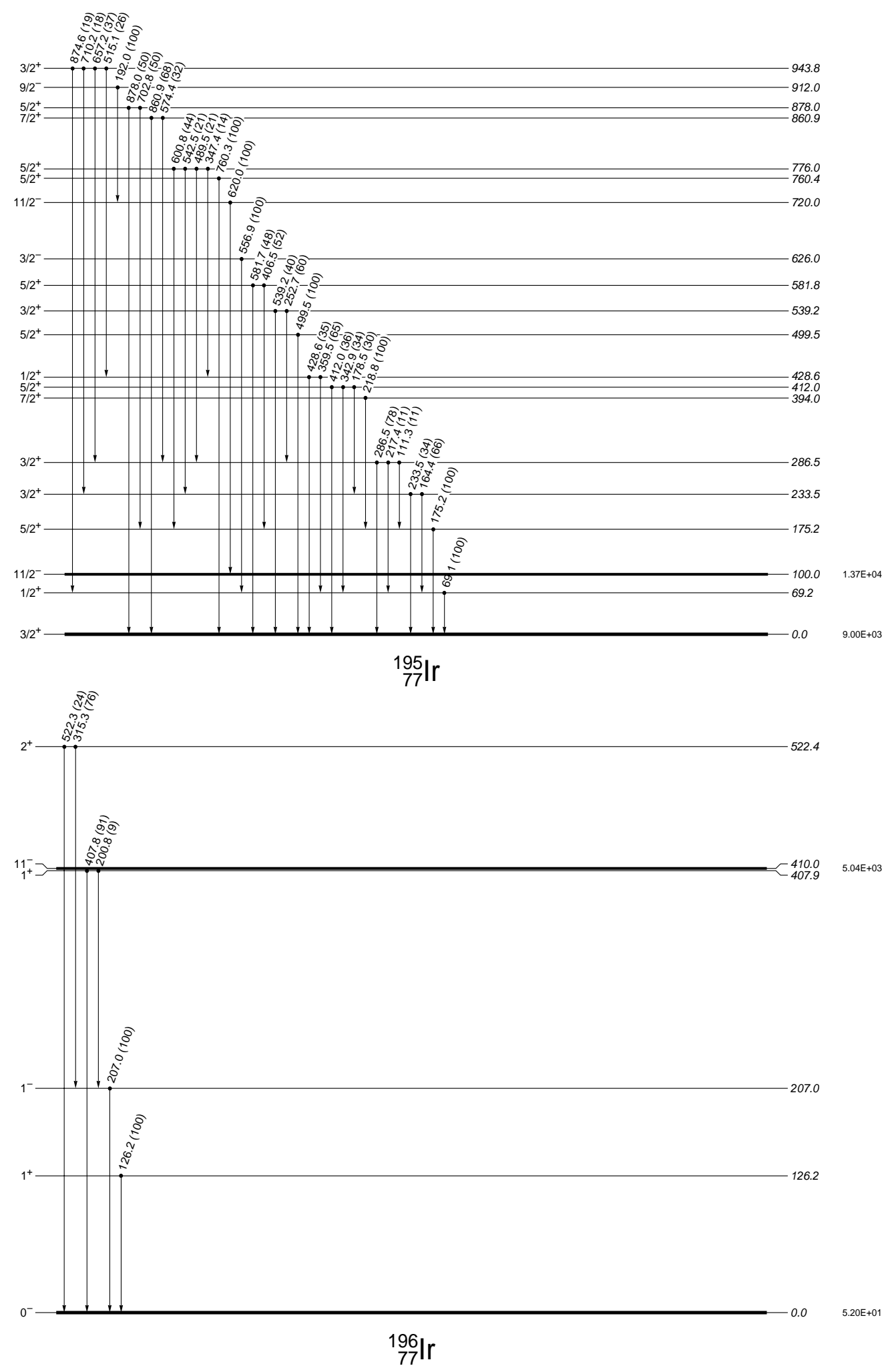

Fig. 24.- Adopted level schemes for ${ }^{195} \mathrm{Ir}$ and ${ }^{196} \mathrm{Ir}$. 

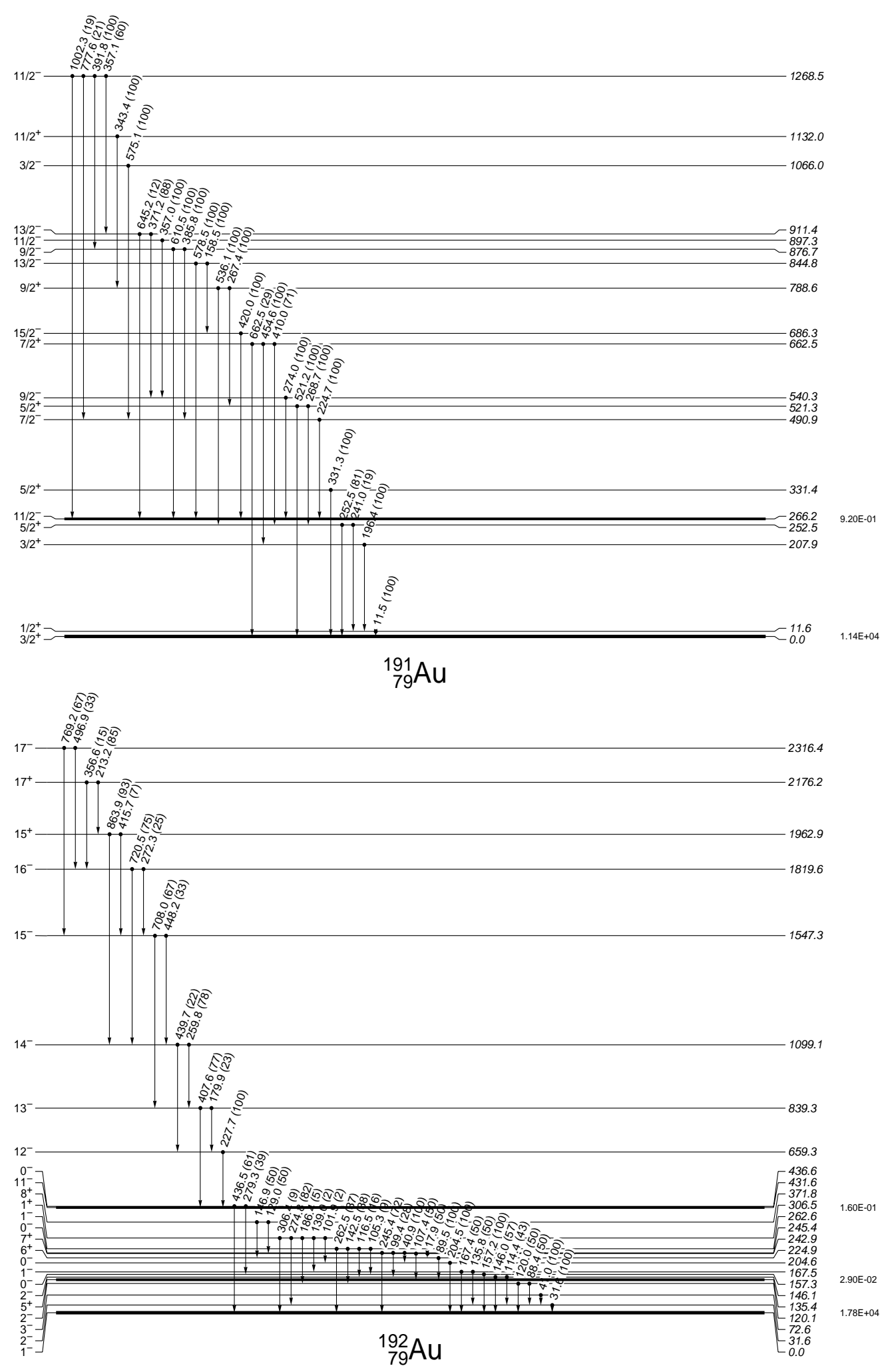

Fig. 25.- Adopted level schemes for ${ }^{191} \mathrm{Au}$ and ${ }^{192} \mathrm{Au}$. 


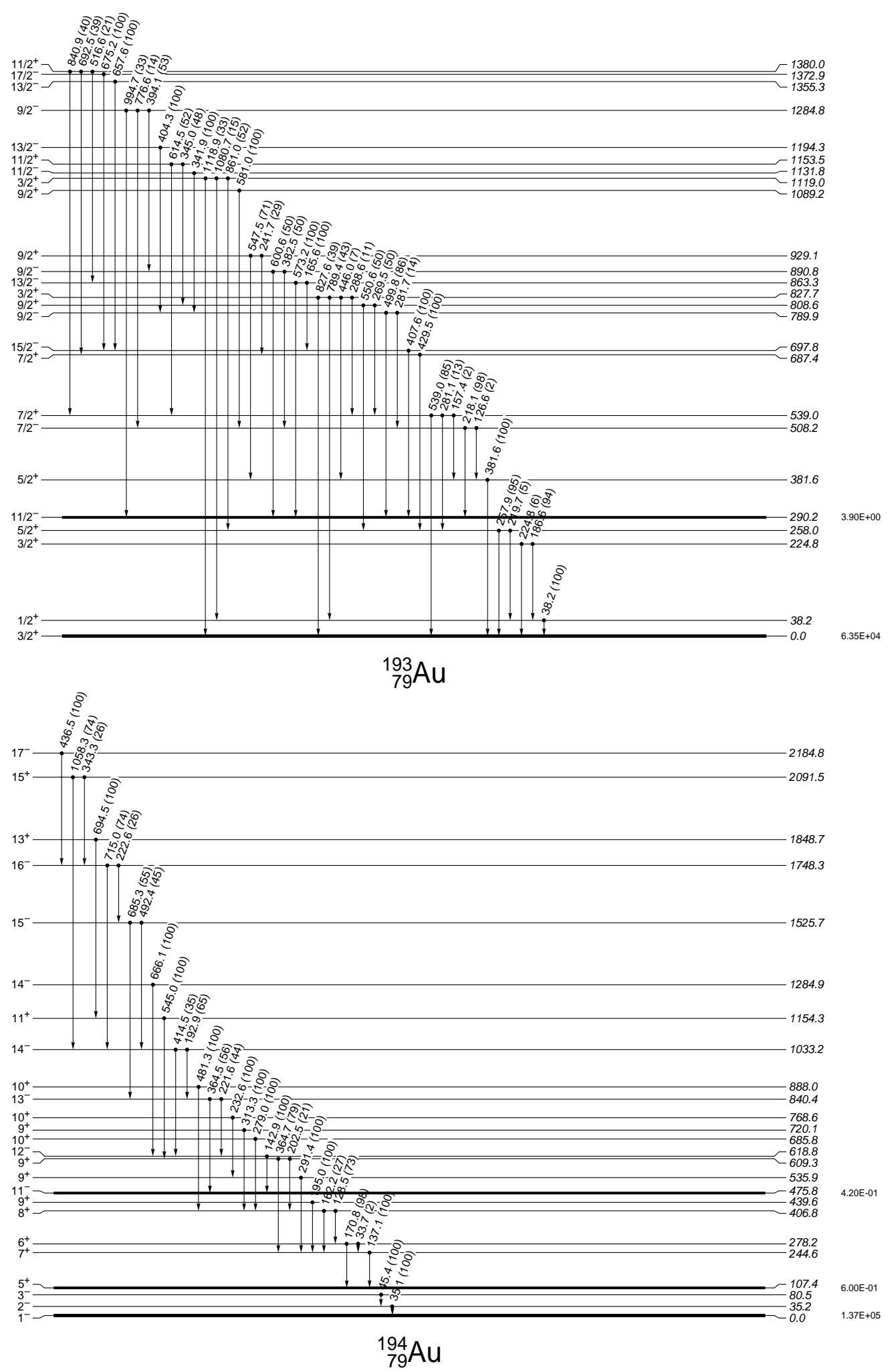

Fig. 26. - Adopted level schemes for ${ }^{193} \mathrm{Au}$ and ${ }^{194} \mathrm{Au}$. 

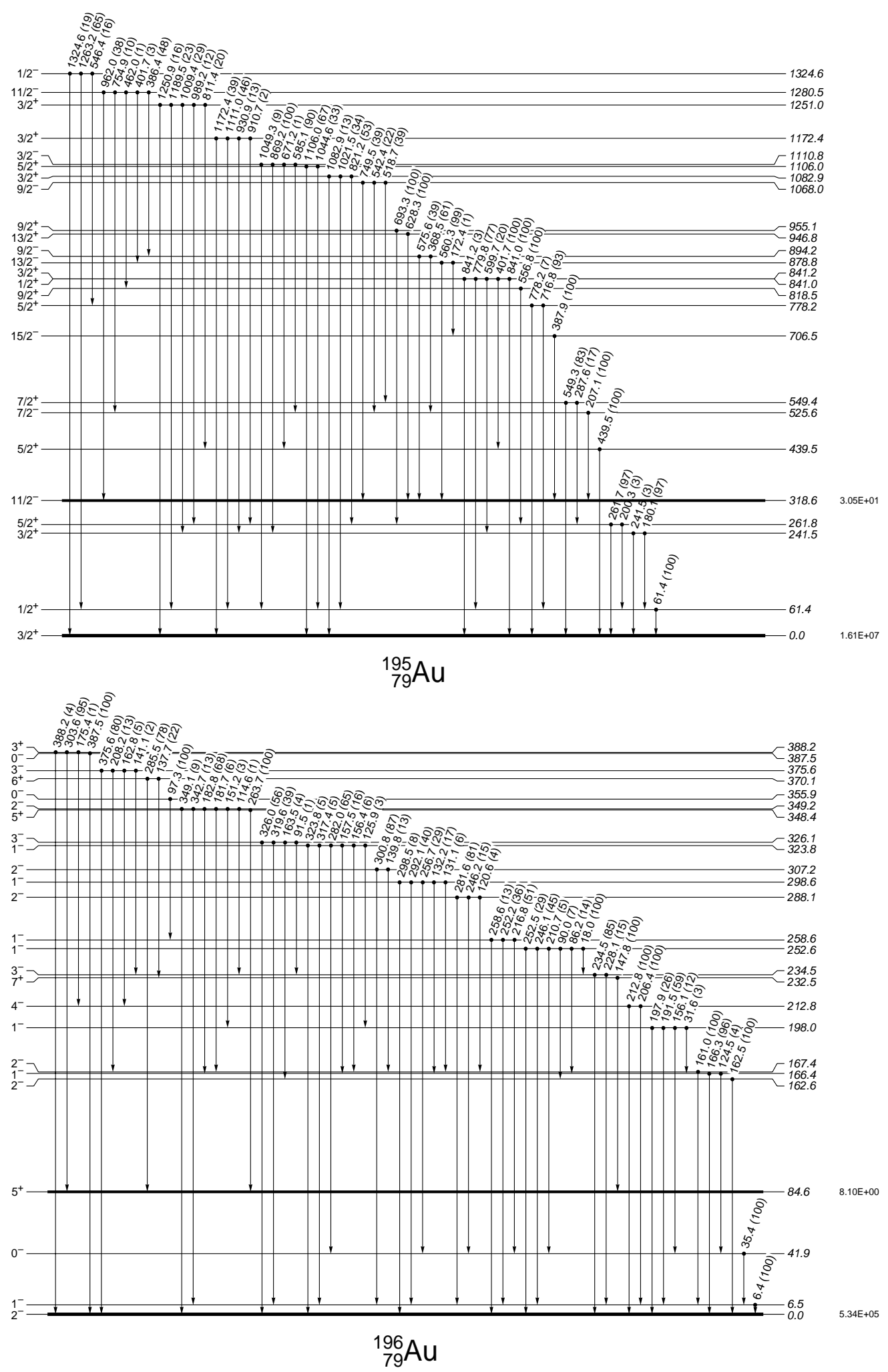

Fig. 27.- Adopted level schemes for ${ }^{195} \mathrm{Au}$ and ${ }^{196} \mathrm{Au}$. 


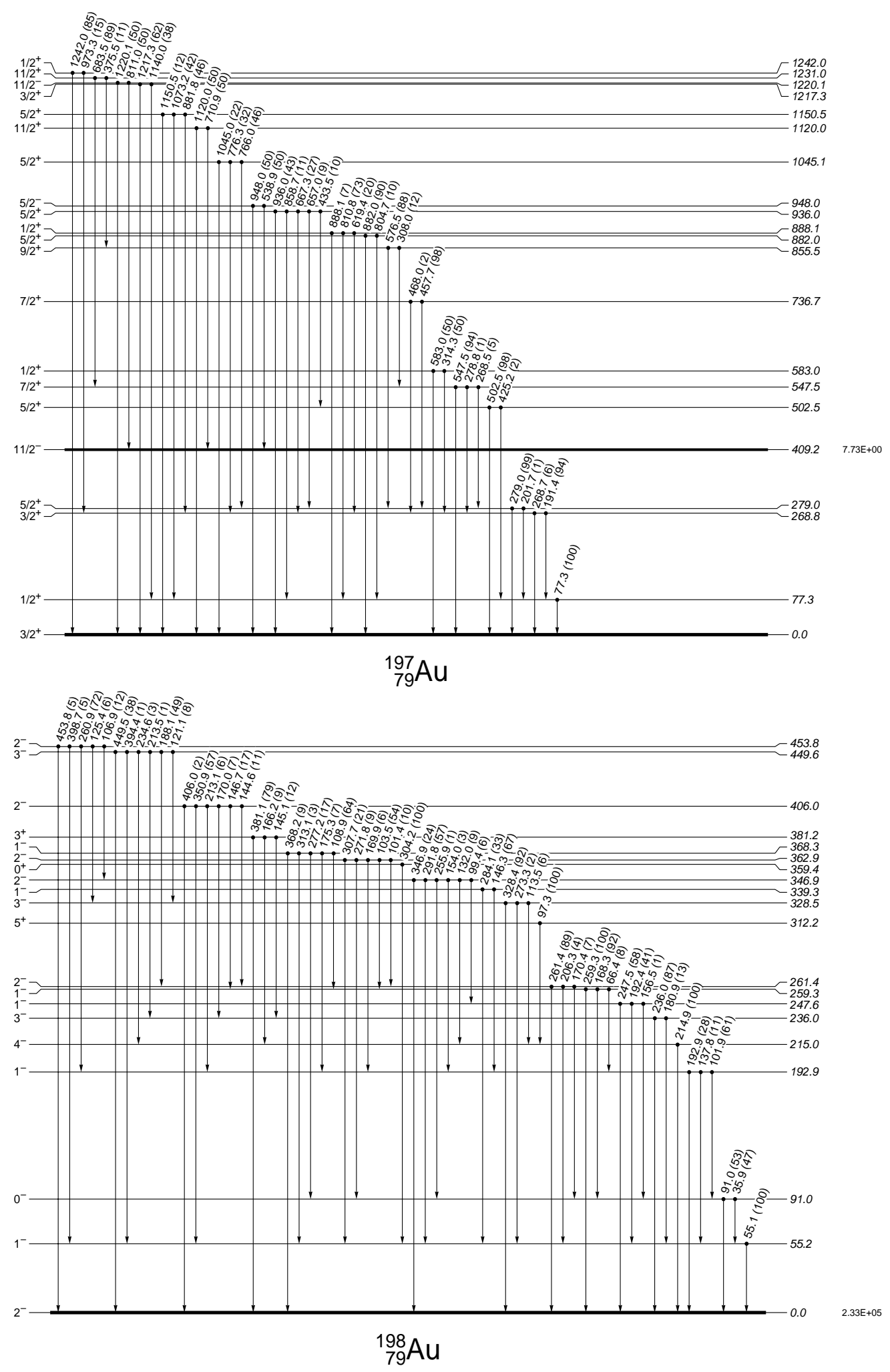

Fig. 28. - Adopted level schemes for ${ }^{197} \mathrm{Au}$ and ${ }^{198} \mathrm{Au}$. 

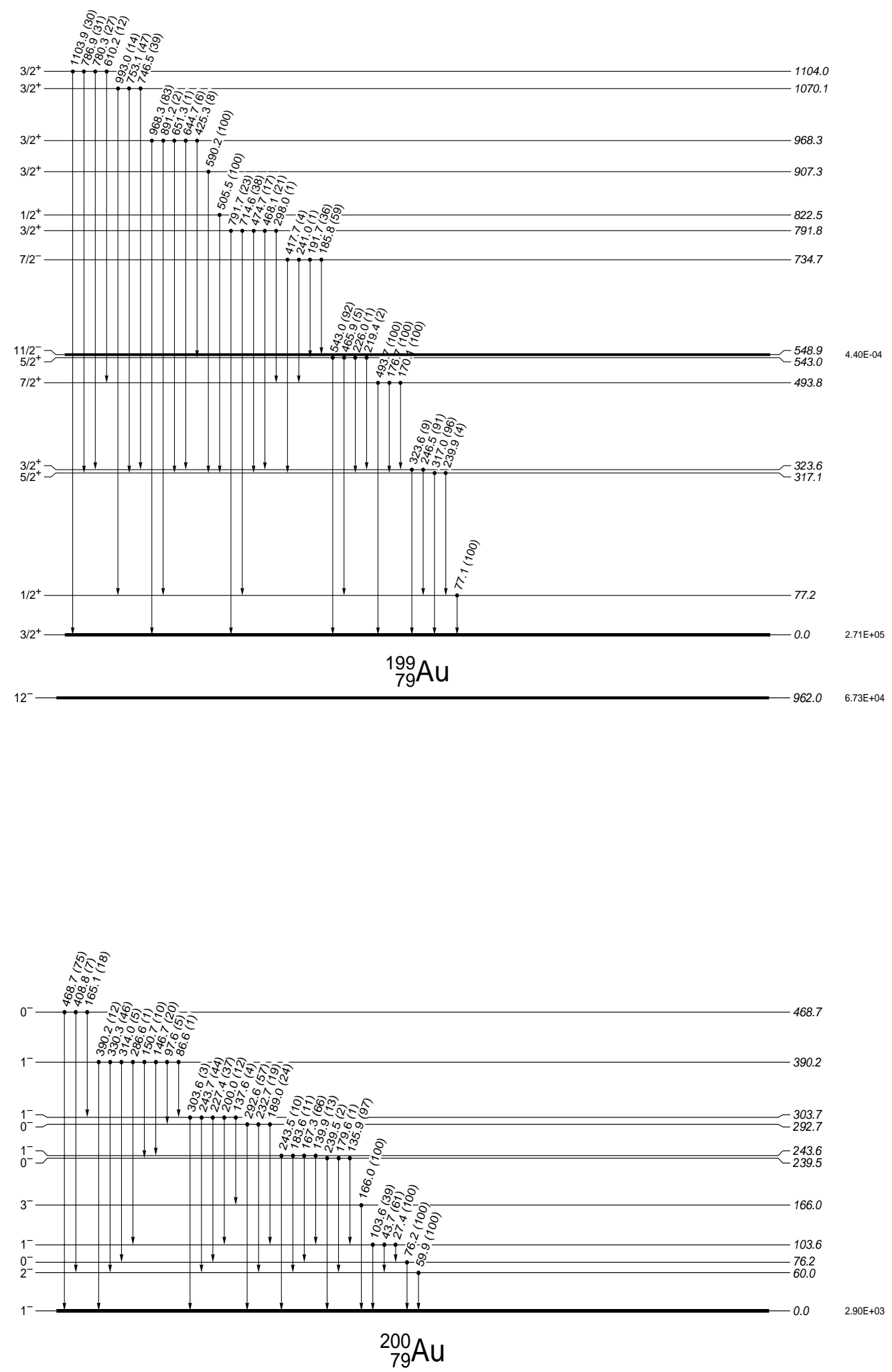

Fig. 29.- Adopted level schemes for ${ }^{199} \mathrm{Au}$ and ${ }^{200} \mathrm{Au}$. 


\section{A.4. Level Density Parameters}

Here we present the level density parameters, as described in section 3.3, for each nucleus considered as a target, compound nucleus, or possible exit channel in this study. The first column lists the nuclei included. The second column is the asymptotic level density parameter $\tilde{a}$ in $\mathrm{MeV}^{-1}$. Column three is the backshift $\Delta$ in $\mathrm{MeV}$. Columns four and five are the shell correction $\delta W$ in $\mathrm{MeV}$ and a flag that indicates whether $\tilde{a}$ is based on an experimentally measured resonance spacing $(\mathrm{x})$ or is taken from systematics (s). Column six gives the matching energy $E_{x}$. Columns seven, eight, and nine are the constant temperature parameters: the spin cutoff parameter $\sigma\left(E_{x}\right)$, the nuclear temperature $T$, and $E_{0}$ (both in $\mathrm{MeV}$ ) all evaluated at $E_{x}$. The last column indicates the number of excited states $N$ to which the level density was fit, which is also the number of excited states included in our Hauser-Feshbach calculations.

Table 7: Level Density Parameters

\begin{tabular}{|c|c|c|c|c|c|c|c|c|c|}
\hline Target & $\tilde{a}$ & $\overline{\bar{\Delta}}$ & $\overline{\bar{\delta} \delta W}$ & $\mathrm{x} / \mathrm{s}$ & 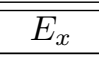 & $\overline{c \sigma\left(E_{x}\right)}$ & $\bar{T}$ & 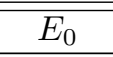 & $\overline{N N}$ \\
\hline${ }^{179} \mathrm{~W}$ & 20.614 & .193 & -.790 & & 3.440 & 4.511 & .496 & -2.114 & 7 \\
\hline${ }^{180} \mathrm{~W}$ & 20.812 & .855 & -1.110 & & 3.021 & 4.100 & .430 & -.659 & 13 \\
\hline${ }^{181} \mathrm{~W}$ & 21.009 & .187 & -1.500 & $*$ & 3.576 & 4.582 & .508 & -2.192 & 18 \\
\hline${ }^{182} \mathrm{~W}$ & 21.209 & 1.030 & -1.550 & & 3.470 & 4.207 & .449 & -.666 & 21 \\
\hline${ }^{183} \mathrm{~W}$ & 20.648 & .215 & -1.820 & $*$ & 3.887 & 4.651 & .534 & -2.349 & 10 \\
\hline${ }^{184} \mathrm{~W}$ & 21.201 & .988 & -1.790 & * & 4.383 & 4.572 & .510 & -1.382 & 21 \\
\hline${ }^{185} \mathrm{~W}$ & 21.789 & .143 & -2.010 & * & 4.203 & 4.840 & .540 & -2.700 & 7 \\
\hline${ }^{186} \mathrm{~W}$ & 22.007 & 1.030 & -2.040 & & 4.379 & 4.656 & .500 & -1.298 & 23 \\
\hline${ }^{187} \mathrm{~W}$ & 23.108 & .205 & -2.320 & * & 3.432 & 4.686 & .482 & -2.028 & 5 \\
\hline${ }^{188} \mathrm{~W}$ & 22.407 & .788 & -2.310 & & 3.487 & 4.454 & .461 & -1.069 & 0 \\
\hline${ }^{189} \mathrm{~W}$ & 22.608 & -.417 & -2.610 & & 2.877 & 4.709 & .497 & -2.678 & 0 \\
\hline${ }^{190} \mathrm{~W}$ & 22.809 & .550 & -2.570 & & 3.839 & 4.673 & .493 & -1.712 & 0 \\
\hline${ }^{191} \mathrm{~W}$ & 23.010 & .470 & -3.210 & & 3.755 & 4.700 & .500 & -1.753 & 0 \\
\hline${ }^{181} \mathrm{Re}$ & 21.010 & .870 & -1.230 & & 5.223 & 4.898 & .556 & -2.242 & 20 \\
\hline${ }^{182} \mathrm{Re}$ & 21.209 & -.960 & -1.660 & & .000 & 3.344 & .342 & -1.681 & 2 \\
\hline${ }^{183} \mathrm{Re}$ & 21.408 & .265 & -1.840 & & 2.833 & 4.313 & .459 & -1.513 & 23 \\
\hline${ }^{184} \mathrm{Re}$ & 21.607 & -.485 & -2.060 & & 1.846 & 4.213 & .444 & -2.091 & 0 \\
\hline${ }^{185} \mathrm{Re}$ & 21.807 & .115 & -2.020 & & 2.200 & 4.141 & .425 & -1.324 & 3 \\
\hline${ }^{186} \mathrm{Re}$ & 21.962 & -.485 & -2.270 & $*$ & 1.841 & 4.255 & .442 & -2.083 & 5 \\
\hline${ }^{187} \mathrm{Re}$ & 22.207 & .070 & -2.230 & & 2.381 & 4.271 & .438 & -1.520 & 3 \\
\hline${ }^{188} \mathrm{Re}$ & 22.634 & -.508 & -2.570 & $*$ & 2.335 & 4.506 & .470 & -2.455 & 0 \\
\hline${ }^{189} \mathrm{Re}$ & 22.608 & -.005 & -2.550 & & 1.926 & 4.093 & .412 & -1.329 & 0 \\
\hline${ }^{190} \mathrm{Re}$ & 22.809 & -.478 & -2.940 & & 1.163 & 3.920 & .395 & -1.606 & 3 \\
\hline${ }^{191} \mathrm{Re}$ & 23.010 & -.208 & -3.220 & & 2.890 & 4.629 & .490 & -2.302 & 0 \\
\hline${ }^{192} \mathrm{Re}$ & 23.212 & -.218 & -3.850 & & 3.063 & 4.668 & .507 & -2.400 & 0 \\
\hline${ }^{193} \mathrm{Re}$ & 23.414 & .150 & -3.940 & & 3.427 & 4.678 & .505 & -2.025 & 0 \\
\hline${ }^{194} \mathrm{Re}$ & 23.616 & -.445 & -4.130 & & 2.828 & 4.680 & .505 & -2.606 & 0 \\
\hline${ }^{182} \mathrm{Os}$ & 21.209 & .352 & -.880 & & .884 & 2.919 & .299 & -.154 & 4 \\
\hline${ }^{183} \mathrm{Os}$ & 21.408 & .775 & -1.250 & & 5.471 & 5.034 & .567 & -2.600 & 34 \\
\hline${ }^{184} \mathrm{Os}$ & 21.607 & .748 & -1.510 & & 2.732 & 4.054 & .413 & -.632 & 4 \\
\hline${ }^{185} \mathrm{Os}$ & 21.807 & .147 & -1.710 & & 3.801 & 4.763 & .514 & -2.420 & 11 \\
\hline${ }^{186} \mathrm{Os}$ & 22.007 & 1.055 & -1.730 & & 4.175 & 4.594 & .482 & -1.124 & 5 \\
\hline
\end{tabular}

Continued on next page... 
Table 7: (continued)

\begin{tabular}{|c|c|c|c|c|c|c|c|c|c|}
\hline Target & $\overline{\tilde{a}}$ & $\overline{\Delta \Delta}$ & $\overline{\delta \delta W}$ & $\mathrm{x} / \mathrm{s}$ & $\overline{E_{x}}$ & $\sigma\left(E_{x}\right)$ & $\bar{T}$ & $\overline{E_{0}}$ & $\bar{N}$ \\
\hline${ }^{187} \mathrm{Os}$ & 21.888 & .120 & -1.980 & * & 4.758 & 5.062 & .566 & -3.150 & 4 \\
\hline${ }^{188} \mathrm{Os}$ & 22.491 & 1.168 & -1.900 & $*$ & 4.258 & 4.613 & .477 & -.984 & 25 \\
\hline${ }^{189} \mathrm{Os}$ & 22.282 & .095 & -2.240 & $*$ & 3.984 & 4.862 & .527 & -2.612 & 8 \\
\hline${ }^{190} \mathrm{Os}$ & 22.712 & 1.185 & -2.300 & $*$ & 4.461 & 4.710 & .490 & -1.082 & 14 \\
\hline${ }^{191} \mathrm{Os}$ & 23.047 & .145 & -2.890 & $*$ & 3.494 & 4.748 & .498 & -2.142 & 1 \\
\hline${ }^{192} \mathrm{Os}$ & 23.212 & 1.215 & -3.290 & & 4.751 & 4.773 & .511 & -1.177 & 36 \\
\hline${ }^{193} \mathrm{Os}$ & 23.669 & .060 & -3.940 & * & 3.419 & 4.720 & .506 & -2.171 & 9 \\
\hline${ }^{194} \mathrm{Os}$ & 23.616 & 1.015 & -4.180 & & 5.302 & 5.012 & .556 & -1.814 & 1 \\
\hline${ }^{195} \mathrm{Os}$ & 23.818 & .250 & -4.610 & & 3.519 & 4.658 & .510 & -1.877 & 0 \\
\hline${ }^{196} \mathrm{Os}$ & 24.021 & 1.177 & -4.600 & & 4.442 & 5.101 & .507 & -.950 & 0 \\
\hline${ }^{184} \mathrm{Ir}$ & 21.607 & -.905 & -1.000 & & .000 & 3.356 & .323 & -1.589 & 28 \\
\hline${ }^{185} \mathrm{Ir}$ & 21.807 & .802 & -1.370 & & 5.555 & 5.114 & .565 & -2.609 & 17 \\
\hline${ }^{186} \mathrm{Ir}$ & 22.007 & -1.100 & -1.560 & & .000 & 3.523 & .342 & -1.898 & 22 \\
\hline${ }^{187} \mathrm{Ir}$ & 22.207 & .338 & -1.390 & & 3.654 & 4.735 & .486 & -2.001 & 28 \\
\hline${ }^{188} \mathrm{Ir}$ & 22.407 & -.693 & -1.550 & & .079 & 3.290 & .311 & -1.301 & 19 \\
\hline${ }^{189} \mathrm{Ir}$ & 22.608 & .083 & -1.650 & & 2.124 & 4.183 & .407 & -1.334 & 39 \\
\hline${ }^{190} \mathrm{Ir}$ & 22.809 & -.758 & -2.160 & & 1.964 & 4.509 & .455 & -2.638 & 49 \\
\hline${ }^{191} \mathrm{Ir}$ & 23.010 & .080 & -2.440 & & 2.720 & 4.473 & .451 & -1.733 & 32 \\
\hline${ }^{192} \mathrm{Ir}$ & 23.410 & -.698 & -3.020 & $*$ & 2.960 & 4.874 & .511 & -3.194 & 49 \\
\hline${ }^{193} \mathrm{Ir}$ & 22.969 & .025 & -3.510 & $*$ & 3.080 & 4.605 & .492 & -2.024 & 42 \\
\hline${ }^{194} \mathrm{Ir}$ & 23.399 & -.575 & -4.100 & $*$ & 3.165 & 4.834 & .532 & -3.045 & 45 \\
\hline${ }^{195} \mathrm{Ir}$ & 23.818 & -.077 & -4.570 & & 1.958 & 4.129 & .438 & -1.422 & 19 \\
\hline${ }^{196} \mathrm{Ir}$ & 24.021 & -.572 & -5.070 & & .000 & 2.978 & .347 & -1.135 & 5 \\
\hline${ }^{197} \mathrm{Ir}$ & 24.224 & -.075 & -5.320 & & 2.541 & 4.750 & .482 & -1.750 & 0 \\
\hline${ }^{198} \mathrm{Ir}$ & 24.427 & -.560 & -6.420 & & 2.698 & 4.891 & .534 & -2.544 & 0 \\
\hline${ }^{189} \mathrm{Pt}$ & 22.608 & -.105 & -.500 & & 3.533 & 5.429 & .487 & -2.737 & 2 \\
\hline${ }^{190} \mathrm{Pt}$ & 22.809 & 1.200 & -.950 & & 4.087 & 5.112 & .448 & -.846 & 13 \\
\hline${ }^{191} \mathrm{Pt}$ & 23.010 & -.080 & -1.420 & & 2.816 & 5.102 & .452 & -2.115 & 19 \\
\hline${ }^{192} \mathrm{Pt}$ & 23.212 & 1.217 & -1.980 & & 4.720 & 5.331 & .491 & -1.234 & 22 \\
\hline${ }^{193} \mathrm{Pt}$ & 23.426 & -.065 & -2.580 & * & 3.601 & 5.367 & .505 & -2.598 & 9 \\
\hline${ }^{194} \mathrm{Pt}$ & 23.616 & 1.180 & -3.280 & & 4.549 & 5.189 & .497 & -1.100 & 14 \\
\hline${ }^{195} \mathrm{Pt}$ & 21.048 & .027 & -4.000 & $*$ & 4.397 & 5.337 & .597 & -2.847 & 9 \\
\hline${ }^{196} \mathrm{Pt}$ & 23.738 & 1.140 & -4.750 & $*$ & 4.934 & 5.220 & .540 & -1.313 & 26 \\
\hline${ }^{197} \mathrm{Pt}$ & 22.473 & .025 & -5.360 & $*$ & 4.792 & 5.444 & .613 & -2.956 & 5 \\
\hline${ }^{198} \mathrm{Pt}$ & 24.427 & 1.113 & -6.050 & & 5.134 & 5.233 & .563 & -1.353 & 4 \\
\hline${ }^{199} \mathrm{Pt}$ & 26.642 & .018 & -7.110 & * & 6.322 & 5.861 & .634 & -3.583 & 1 \\
\hline${ }^{200} \mathrm{Pt}$ & 24.835 & 1.105 & -7.730 & & 5.266 & 5.039 & .595 & -1.221 & 10 \\
\hline${ }^{201} \mathrm{Pt}$ & 25.039 & -.250 & -8.600 & & 2.996 & 4.639 & .572 & -2.011 & 0 \\
\hline${ }^{190} \mathrm{Au}$ & 22.809 & -.765 & -1.170 & & .000 & 3.653 & .301 & -1.363 & 0 \\
\hline${ }^{191} \mathrm{Au}$ & 23.010 & .147 & -1.650 & & 1.944 & 4.488 & .385 & -1.103 & 15 \\
\hline${ }^{192} \mathrm{Au}$ & 23.212 & -.738 & -2.140 & & .000 & 3.576 & .309 & -1.326 & 2 \\
\hline
\end{tabular}

Continued on next page... 
Table 7: (continued)

\begin{tabular}{|c|c|c|c|c|c|c|c|c|c|}
\hline Target & $\overline{\tilde{a}}$ & 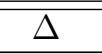 & 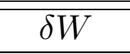 & $\mathrm{x} / \mathrm{s}$ & $E_{x}$ & $\sigma\left(E_{x}\right)$ & $T$ & $E_{0}$ & $N$ \\
\hline${ }^{193} \mathrm{Au}$ & 23.414 & .015 & -2.760 & & 1.436 & 4.168 & .369 & -.973 & 25 \\
\hline${ }^{194} \mathrm{Au}$ & 23.616 & -.603 & -3.330 & & .238 & 3.651 & .331 & -1.251 & 24 \\
\hline${ }^{195} \mathrm{Au}$ & 23.818 & .065 & -4.050 & & 2.026 & 4.459 & .424 & -1.246 & 25 \\
\hline${ }^{196} \mathrm{Au}$ & 24.021 & -.615 & -4.750 & & 1.984 & 4.750 & .473 & -2.307 & 48 \\
\hline${ }^{197} \mathrm{Au}$ & 24.224 & .055 & -5.530 & & 3.035 & 4.844 & .506 & -1.831 & 20 \\
\hline${ }^{198} \mathrm{Au}$ & 24.044 & -.473 & -6.260 & * & 3.879 & 5.301 & .587 & -3.103 & 49 \\
\hline${ }^{199} \mathrm{Au}$ & 24.631 & .035 & -6.900 & & 2.411 & 4.500 & .495 & -1.419 & 13 \\
\hline${ }^{200} \mathrm{Au}$ & 24.835 & -.380 & -7.980 & & 4.645 & 5.331 & .632 & -3.096 & 11 \\
\hline${ }^{201} \mathrm{Au}$ & 25.039 & -.015 & -8.590 & & 3.114 & 4.595 & .567 & -1.721 & 16 \\
\hline${ }^{202} \mathrm{Au}$ & 25.243 & -.230 & -9.440 & & 3.013 & 4.558 & .590 & -1.884 & 0 \\
\hline${ }^{203} \mathrm{Au}$ & 25.447 & -.115 & -10.060 & & 2.965 & 4.412 & .598 & -1.619 & 0 \\
\hline${ }^{204} \mathrm{Au}$ & 25.652 & -.388 & -11.140 & & 2.847 & 4.276 & .633 & -1.771 & 0 \\
\hline${ }^{194} \mathrm{Hg}$ & 23.616 & 1.290 & -3.040 & & 3.420 & 4.590 & .421 & -.157 & 4 \\
\hline${ }^{195} \mathrm{Hg}$ & 23.818 & .035 & -3.670 & & 2.277 & 4.619 & .436 & -1.468 & 12 \\
\hline${ }^{196} \mathrm{Hg}$ & 24.021 & 1.192 & -4.490 & & 3.043 & 4.354 & .422 & -.040 & 26 \\
\hline${ }^{197} \mathrm{Hg}$ & 24.224 & .190 & -5.090 & & 4.367 & 5.357 & .557 & -2.477 & 2 \\
\hline${ }^{198} \mathrm{Hg}$ & 24.427 & 1.120 & -5.960 & & 3.869 & 4.723 & .499 & -.601 & 6 \\
\hline${ }^{199} \mathrm{Hg}$ & 24.631 & .275 & -6.720 & & 5.054 & 5.401 & .604 & -2.537 & 7 \\
\hline${ }^{200} \mathrm{Hg}$ & 24.835 & 1.135 & -7.490 & & 6.037 & 5.377 & .619 & -1.614 & 37 \\
\hline${ }^{201} \mathrm{Hg}$ & 25.039 & .145 & -8.340 & & 6.133 & 5.574 & .667 & -2.955 & 13 \\
\hline${ }^{202} \mathrm{Hg}$ & 25.243 & 1.077 & -9.070 & & 7.162 & 5.476 & .678 & -1.865 & 8 \\
\hline${ }^{203} \mathrm{Hg}$ & 25.447 & .070 & -9.970 & & 6.849 & 5.557 & .707 & -2.853 & 0 \\
\hline${ }^{204} \mathrm{Hg}$ & 25.652 & 1.072 & -10.650 & & 8.859 & 5.690 & .735 & -1.927 & 21 \\
\hline${ }^{205} \mathrm{Hg}$ & 25.857 & .102 & -11.820 & & 2.462 & 3.837 & .628 & -.933 & 5 \\
\hline
\end{tabular}




\section{B. Modeled Cross Sections Compared to Measurements}

B.1. $(\mathbf{n}, \gamma)$
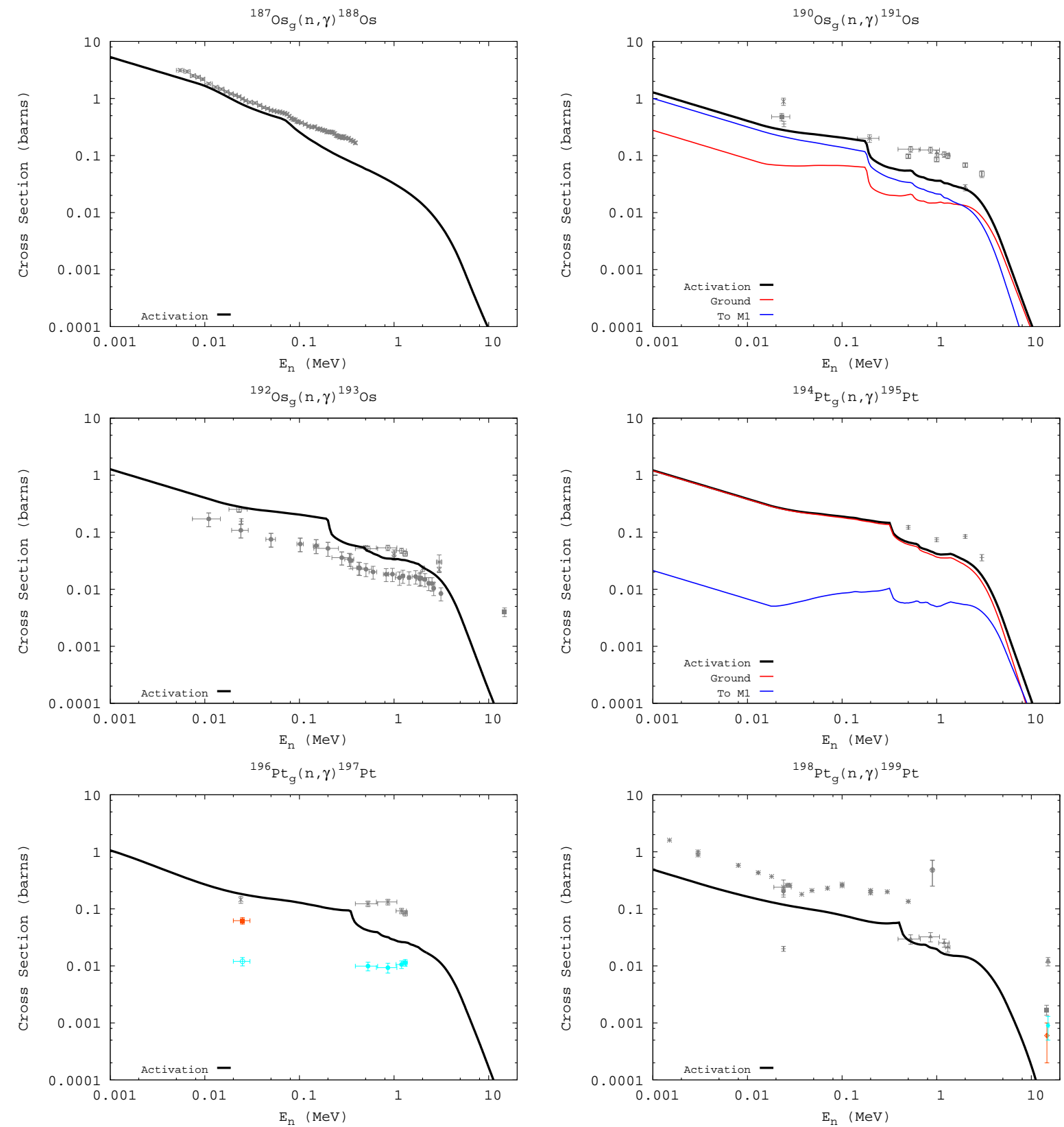

Fig. 30.- Modeled neutron capture cross sections compared to measurement. The data is taken from (EXFOR 2006). The black, red, and blue solid lines represent our modeled cross sections (total, leading to the ground state, and leading to the first isomer, respectively). The grey, orange, and light blue data points are measured cross section data (total, ground state, and first isomer). 


\section{B.2. Maxwellian-averaged $(\mathbf{n}, \gamma)$}
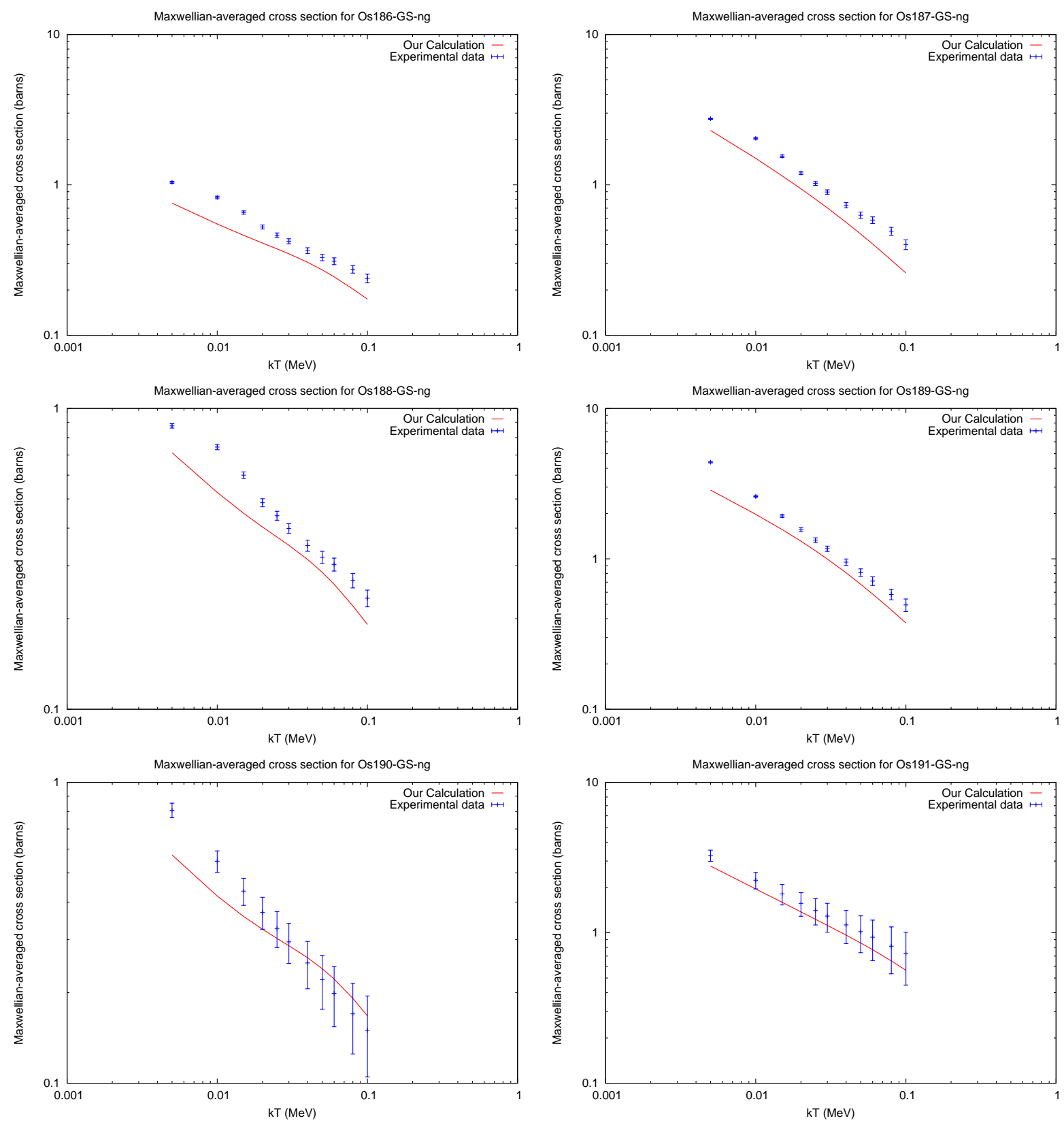

Fig. 31.- Modeled Maxwellian-averaged neutron capture cross sections compared to measurement. The data is taken from (Bao et al. 2000). The solid lines represent our modeled cross sections. The errors on the recommended value for each energy are identical to the stated error at $30 \mathrm{keV}$. 

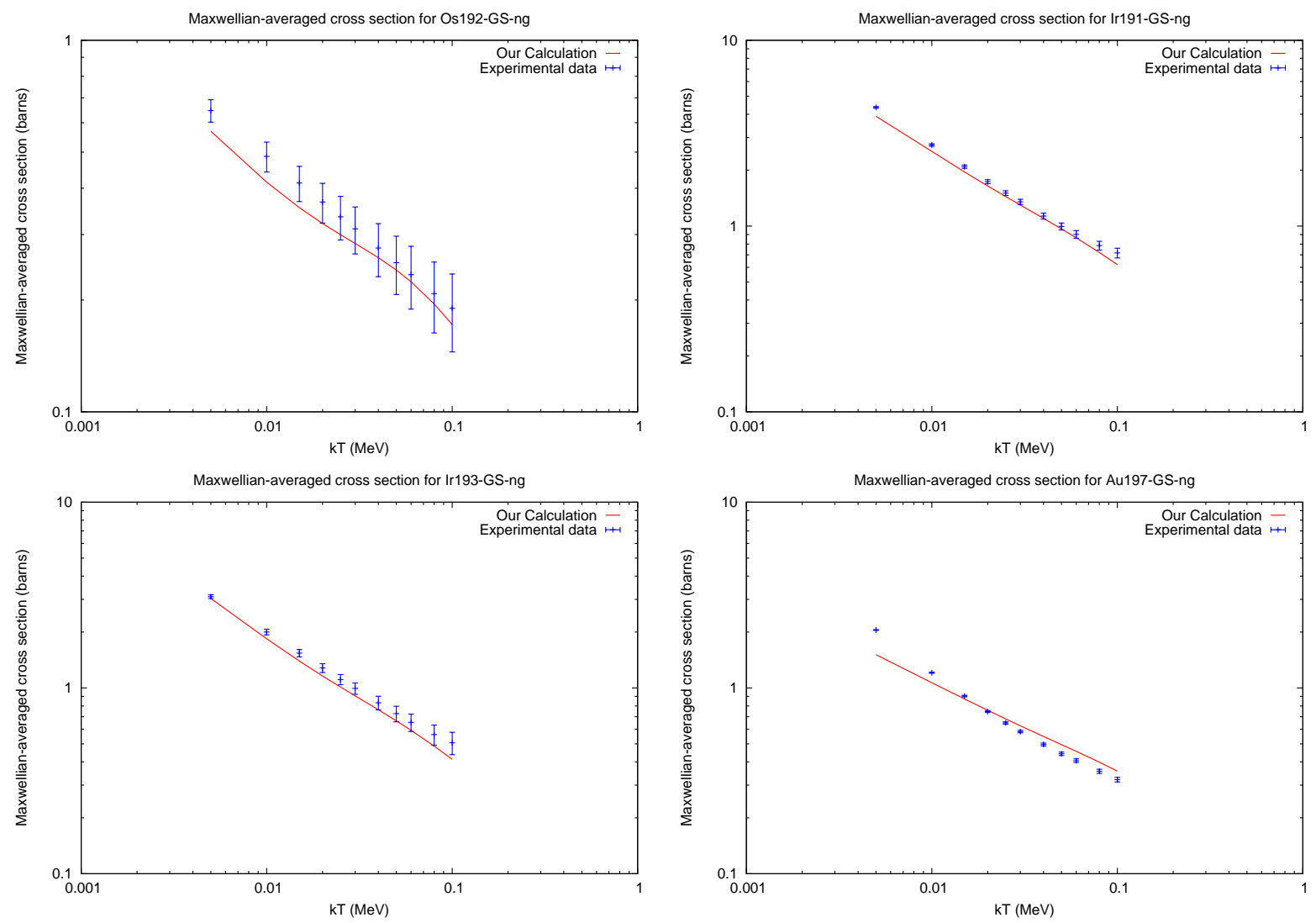

Fig. 31.- (continued) 

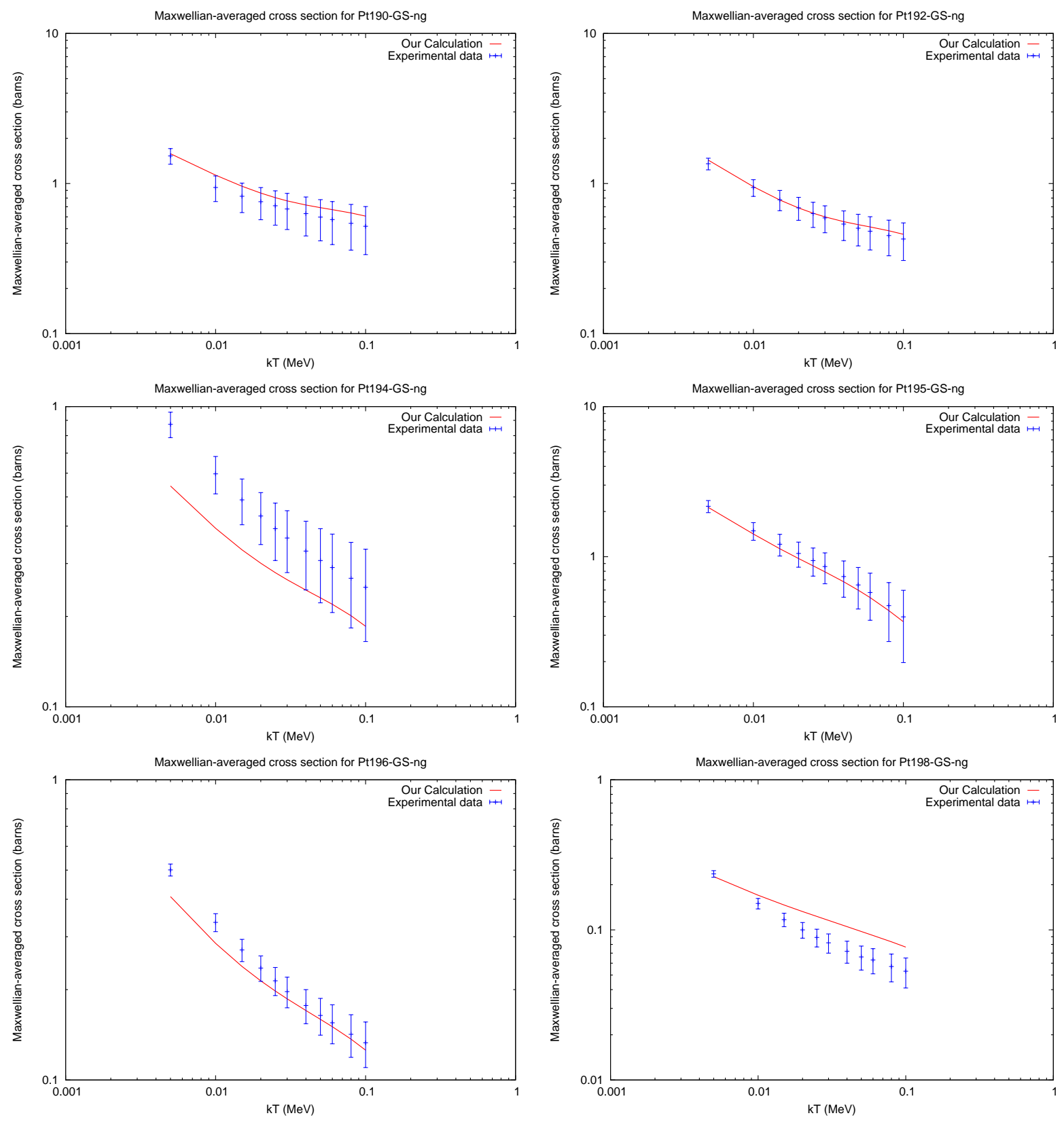

Fig. 31.- (continued) 
B.3. $(\mathrm{n}, 2 \mathrm{n})$
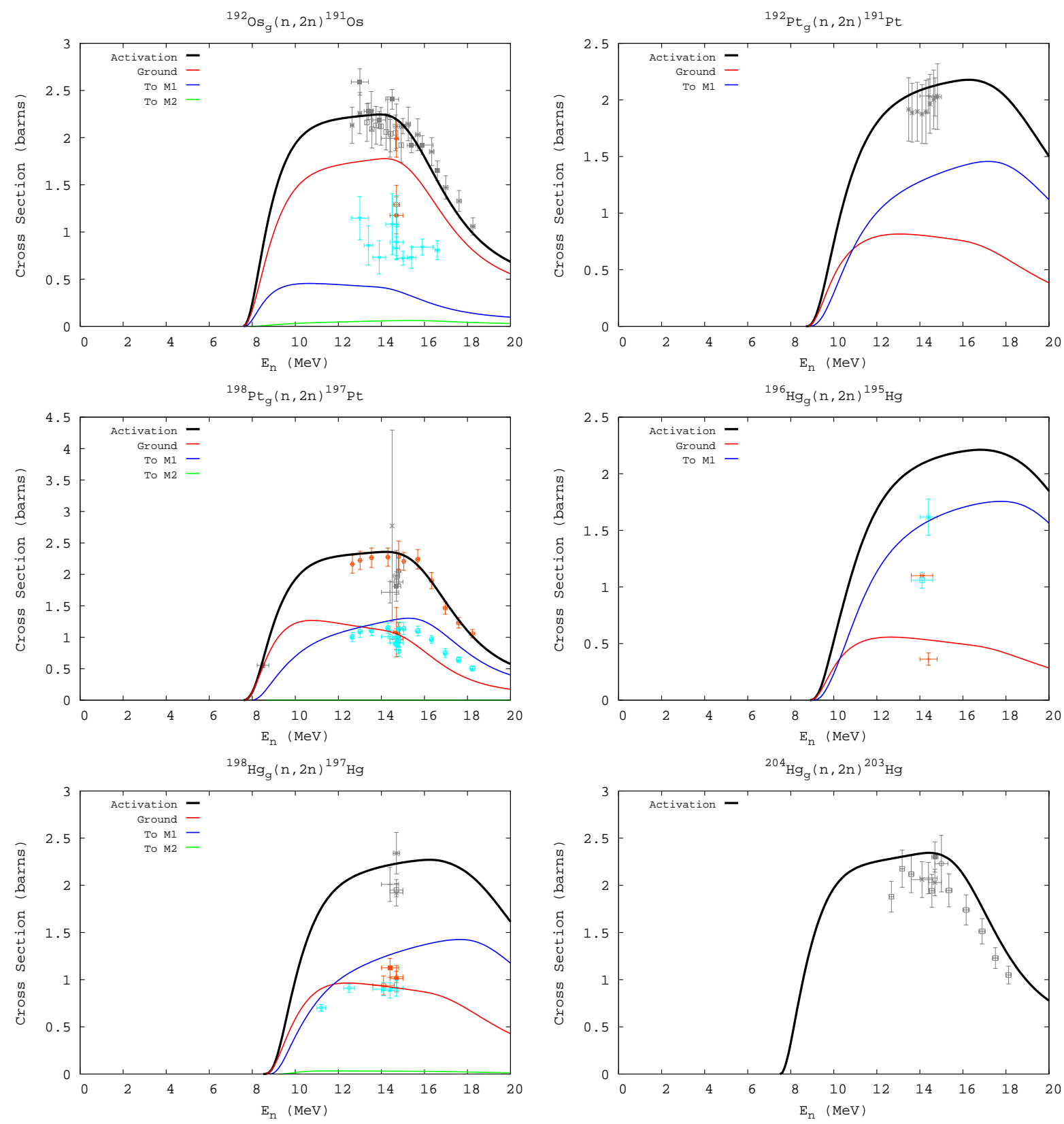

Fig. 32.- Modeled (n,2n) cross sections compared to measurement. The data is taken from (EXFOR 2006). The black, red, and blue solid lines represent our modeled cross sections (total, leading to the ground state, and leading to the first isomer, respectively). The Grey, orange, and light blue data points are measured cross section data (total, ground state, and first isomer). 


\section{B.4. $(\mathrm{n}, \mathrm{p})$}
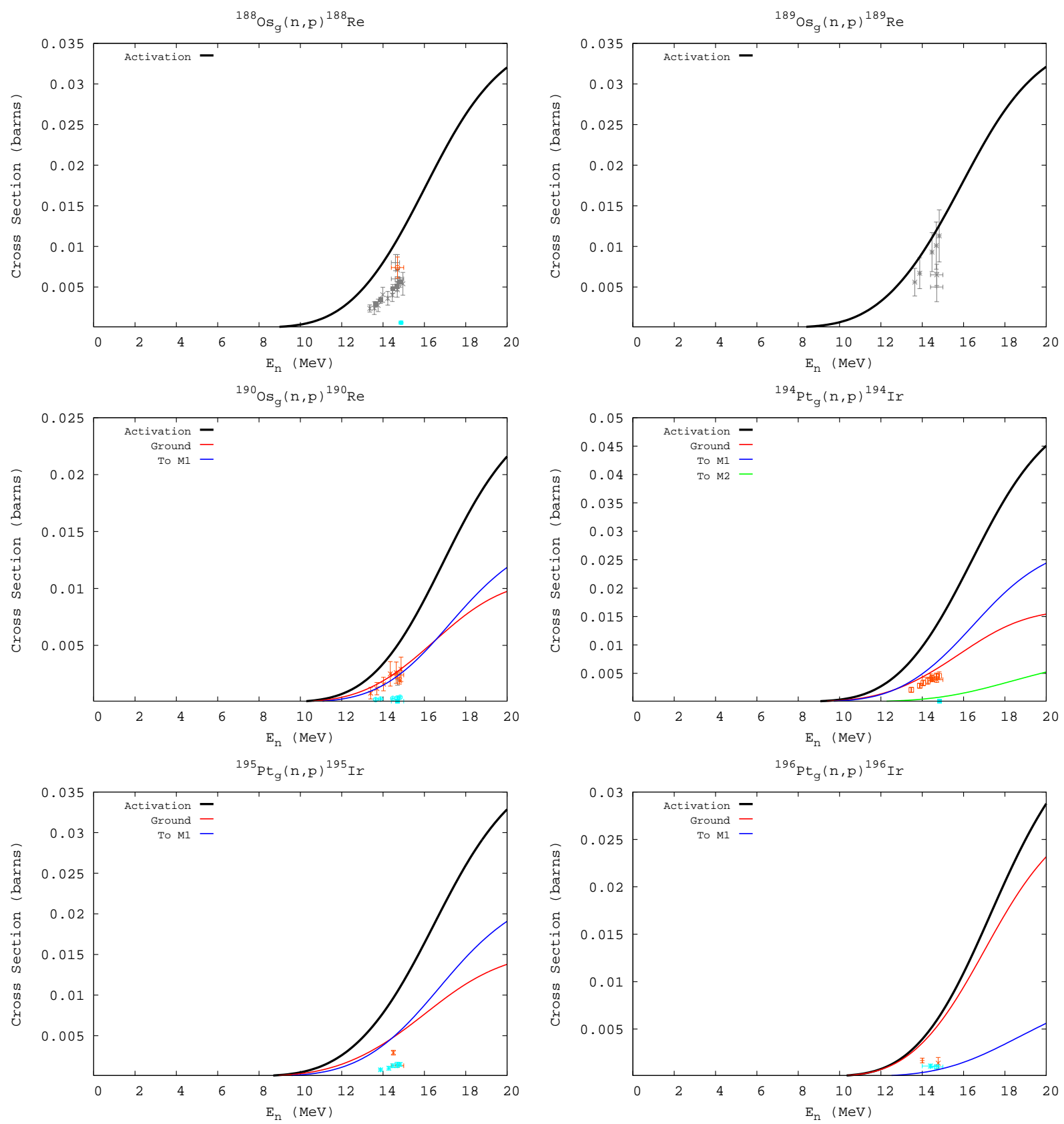

Fig. 33.- Modeled (n,p) cross sections compared to measurement. The data is taken from (EXFOR 2006). The black, red, and blue solid lines represent our modeled cross sections (total, leading to the ground state, and leading to the first isomer, respectively). The grey, orange, and light blue data points are measured cross section data (total, ground state, and first isomer). 


\section{B.5. $(\mathrm{n}, \mathrm{a})$}
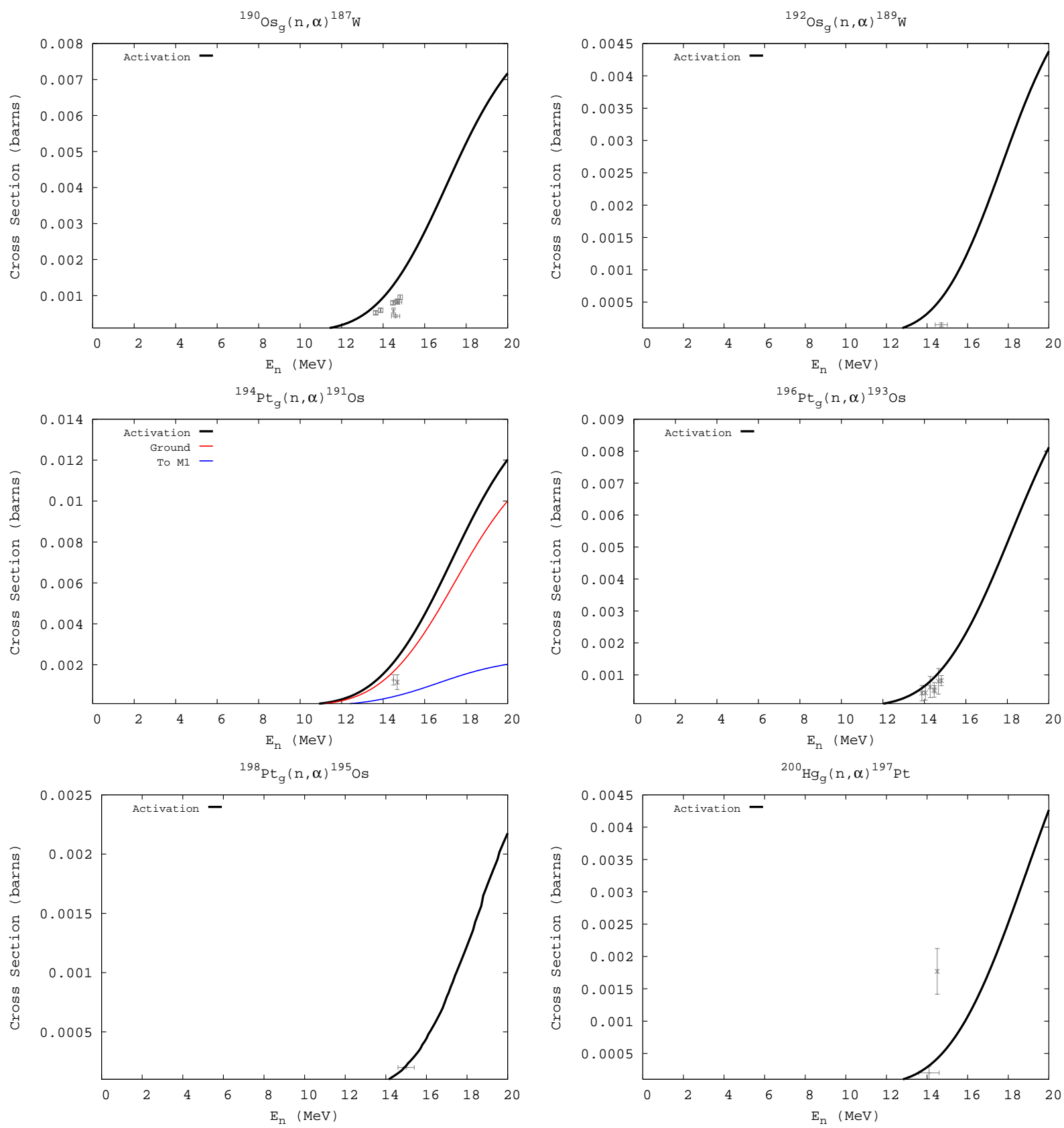

Fig. 34.- Modeled (n, $\alpha$ ) cross sections compared to measurement. The data is taken from (EXFOR 2006). The black, red, and blue solid lines represent our modeled cross sections (total, leading to the ground state, and leading to the first isomer, respectively). The grey, orange, and light blue data points are measured cross section data (total, ground state, and first isomer). 


\section{B.6. Comparison to LANL Radchem}
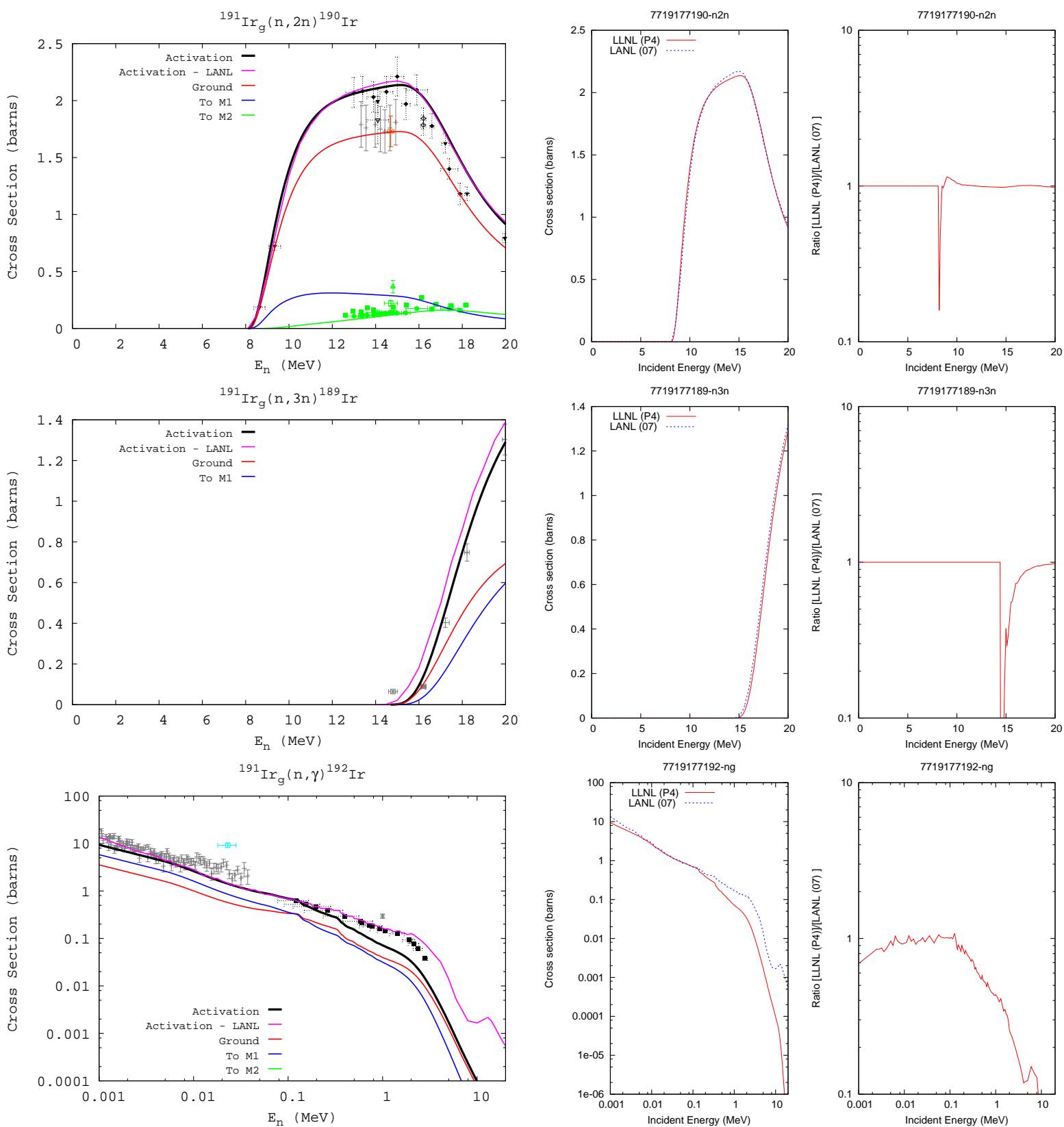

Fig. 35.- Modeled cross sections for ${ }^{191}$ Ir compared to measurement and their LANL counterparts. The data is taken from (EXFOR 2006). The black, red, and blue solid lines represent our modeled cross sections (total, leading to the ground state, and leading to the first isomer, respectively). The Grey, orange, and light blue data points are measured cross section data (total, ground state, and first isomer). The purple line is the LANL cross section. 

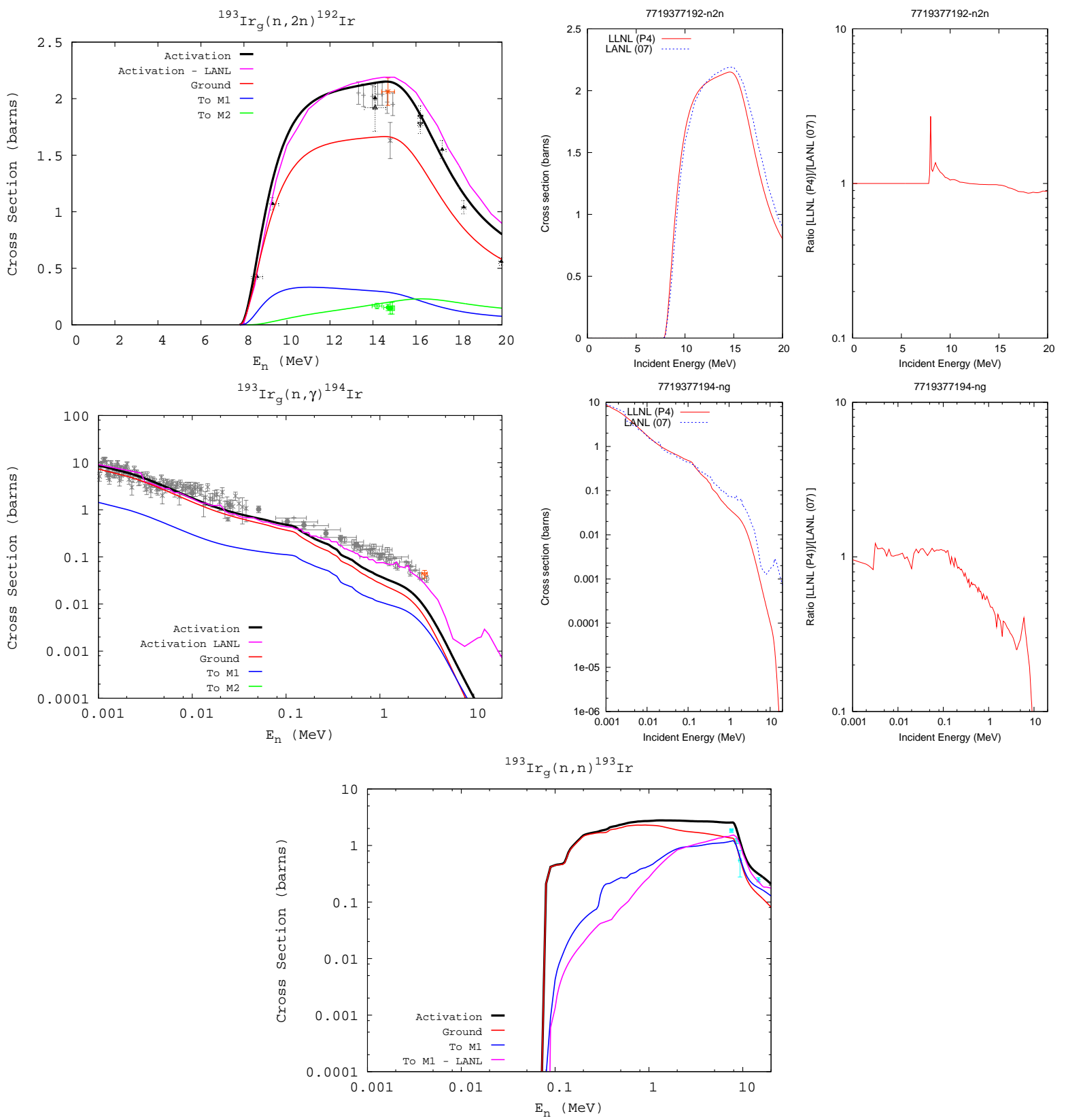

Fig. 36.- Modeled cross sections for ${ }^{193}$ Ir compared to measurement and their LANL counterparts. The data is taken from (EXFOR 2006). The black, red, and blue solid lines represent our modeled cross sections (total, leading to the ground state, and leading to the first isomer, respectively). The Grey, orange, and light blue data points are measured cross section data (total, ground state, and first isomer). The purple line is the LANL cross section. 


\section{Activation Cross Sections by Target}
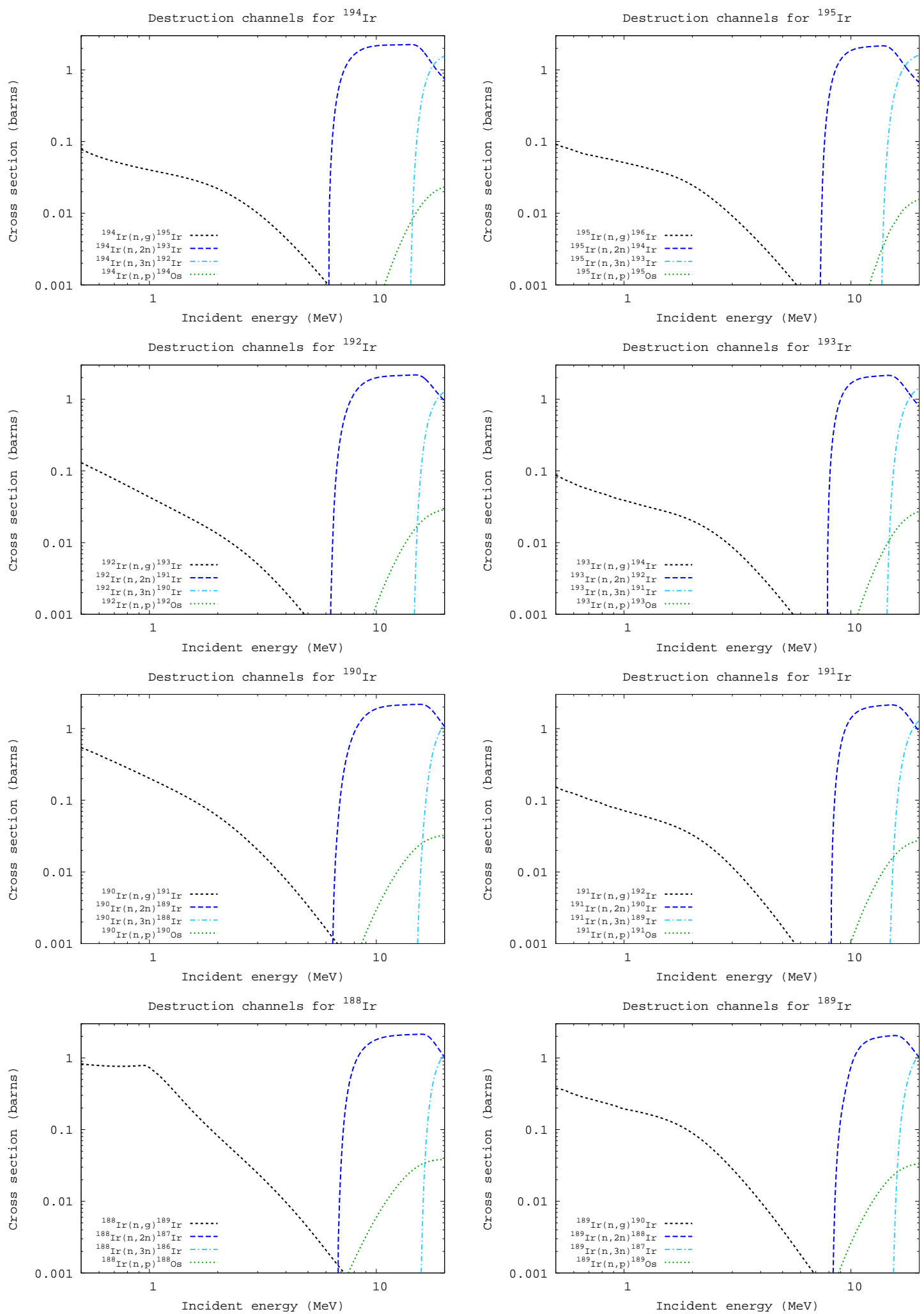

Fig. 37.- Activation cross sections for $\mathrm{Z}=77$ ground state targets of Ir. 

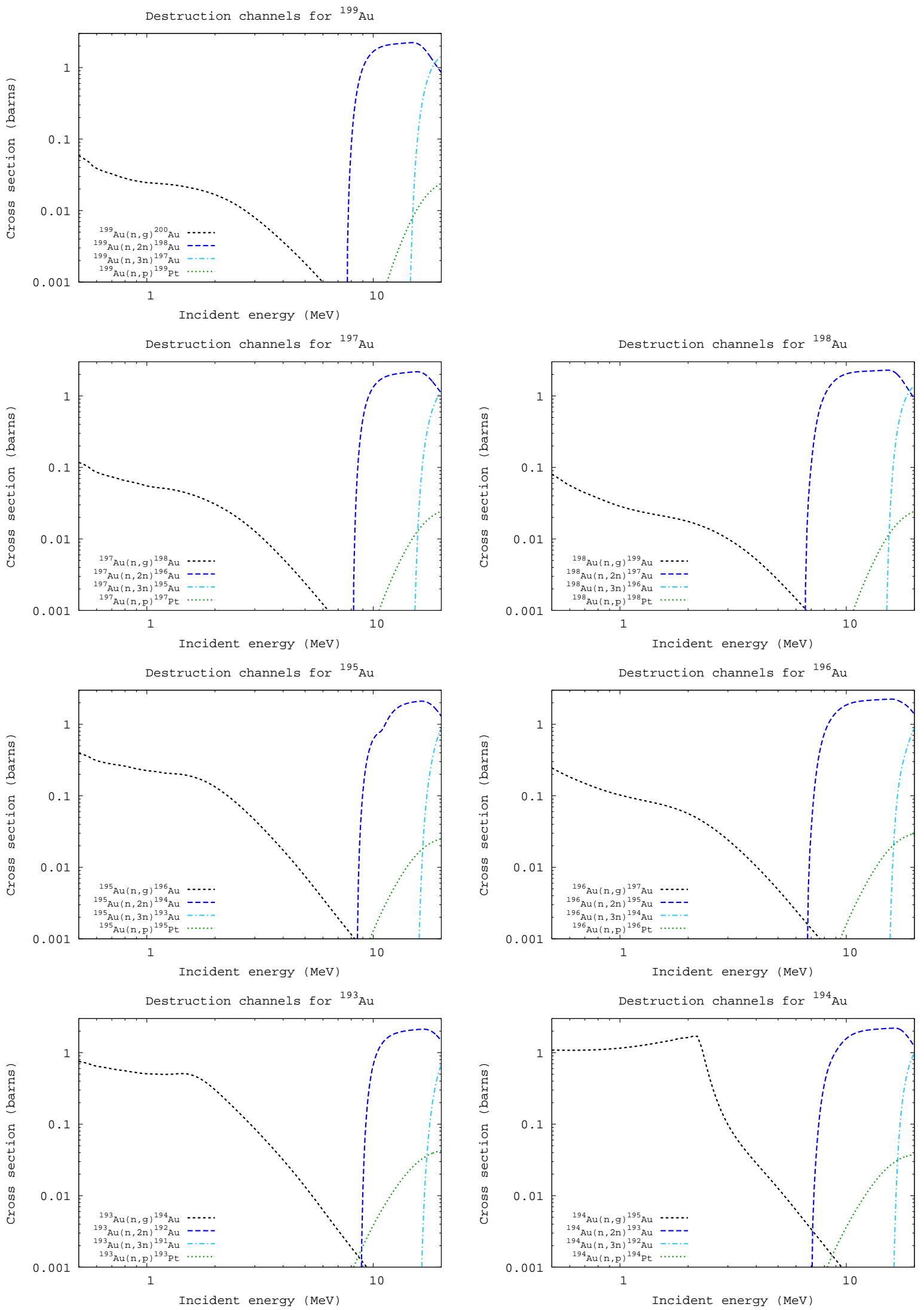

Fig. 38.- Activation cross sections for $\mathrm{Z}=79$ ground state targets of $\mathrm{Au}$. 